\title{
Substrate traits govern the assembly and spatial organization of microbial community engaged in metabolic division of labor
}

\section{Miaoxiao Wang}

Peking University

Xiaoli Chen

Peking University

Yong Nie

Peking University https://orcid.org/0000-0002-5940-1218

Xiao-Lei Wu ( $\nabla$ xiaolei_wu@pku.edu.cn )

Peking University https://orcid.org/0000-0002-9897-6903

\section{Article}

Keywords: metabolic division of labor, microbial community, spatial organization

Posted Date: December 2nd, 2020

DOI: https://doi.org/10.21203/rs.3.rs-110849/v1

License: (c) (i) This work is licensed under a Creative Commons Attribution 4.0 International License. Read Full License 
1 Full title: Substrate traits govern the assembly and spatial organization of microbial

2 community engaged in metabolic division of labor

$3 \quad$ Running title: Substrate traits affect microbial division of labor

4

5

6 Miaoxiao Wang ${ }^{1}$, Xiaoli $\mathrm{Chen}^{1,2}$, Yong $\mathrm{Nie}^{1 \#}$ and Xiao-Lei $\mathrm{Wu}^{1,2,3 \#}$

$7 \quad{ }^{1}$ College of Engineering, Peking University, Beijing 100871, China

$8 \quad{ }^{2}$ Institute of Ocean Research, Peking University, Beijing 100871, China

$9 \quad{ }^{3}$ Institute of Ecology, Peking University, Beijing 100871, China

${ }^{\#}$ Corresponding author: Research Scientist, College of Engineering, Peking University.

Tel: +86 10-62759047; Fax: +86 10-62759047; E-mail: nieyong@pku.edu.cn

${ }^{\#}$ Corresponding author: Professor, College of Engineering, Peking University.

Tel: +86 10-62759047; Fax: +86 10-62759047; E-mail: xiaolei_wu@pku.edu.cn 


\section{Abstract}

Metabolic division of labor (MDOL) is widespread in nature, whereby a complex metabolic pathway is shared between different strains within a community for mutual benefit. However, little is known about how communities engaged in MDOL assemble and spatially organize. We hypothesized that when degradation of an organic compound is carried out via MDOL, substrate concentration and its toxicity modulate the benefit allocation between the two microbial populations, thus governing the assembly of this community. We tested this hypothesis by combining individual-based simulations with pattern formation assays using a synthetic microbial community. We found that while the frequency of the first population increases with an increase in substrate concentration, this increase is capped with an upper bound determined by the biotoxicity of the substrate. In addition, our model showed that substrate concentration and its toxicity affect levels of intermixing between strains. These predictions were quantitatively verified using an engineered system composed of two strains degrading salicylate through MDOL. Our results demonstrate that the structure of the microbial communities can be quantitatively predicted from simple environmental factors, such as substrate concentration and its toxicity, which provides novel perspectives on understanding the assembly of natural communities, as well as insights into how to manage artificial microbial systems. 


\section{Introduction}

In natural environments, microorganisms rarely live autonomously, but interact with other individuals to form complex communities, in which they secrete a variety of toxins to compete with each other, or share metabolites to mutually benefit their survival. Among a variety of modes of microbial interaction, metabolic division of labor (MDOL) is one of the most widespread phenomena, where distinct populations perform different but complementary steps of the same metabolic pathway ${ }^{1-4}$. MDOL controls numerous ecologically and environmentally important biochemical processes. For example, the nitrification pathway is often operated by the collaboration between two populations, in which the first group oxidizes ammonia to nitrite, before being converted to nitrate by another bacterial group ${ }^{5}$. Similarly, during the Deepwater Horizon oil spill Gulf of Mexico (2010), complete degradation of polycyclic aromatic hydrocarbons (PAHs) required the partitioning of key pathway steps into several bacterial groups ${ }^{6}$. Since each population only contains a subset of genetic components required for the overall pathway, MDOL is thought to be a key evolutionary strategy to reduce metabolic burden of each individual ${ }^{7,8}$. In addition, bacteria have been found to use this strategy to reduce the inter-enzyme competition and the inhibitory effects of intermediates, thus improving the systematic metabolic efficiency ${ }^{9}$. Furthermore, MDOL vastly enhances functional differentiation of microbial communities, contributing to genetic diversity in complex ecosystem ${ }^{7,8}$. Nevertheless, how microbial communities engaged in MDOL assemble remains poorly understood. Understanding the assembly rules of communities engaged in MDOL is fundamental for better 
evaluating their contribution to the natural biochemical cycle.

One important aspect of microbial metabolism implemented by MDOL is the degradation of a variety of organic compounds, including PAHs, lignin or chitin. Bacterial degradation of these complex substrates is usually mediated by long metabolic pathways via a number of intermediates. While these pathways often remain intact within one population, they are frequently found segregated across different members within a community, whereby the intermediates are being secreted by one species, before being utilized as substrate by another species for further processing along the same pathway. Typical examples include syringate degradation via sequential crossfeeding between Acetobacterium woodii and Pelobacter acidigallici ${ }^{10}$, phenanthrene degradation between Marinobacter sp. N4 and other PAH-degrading microbes in marine environments ${ }^{11}$, as well as atrazine degradation through MDOL within four bacterial species ${ }^{12}$. Nevertheless, all of these natural communities grow in the environments that may fluctuate over time and vary in space. For example, the concentration of the initial substrate, such as PAHs and other hydrocarbons, is highly fluctuating over time and different sites in the marine ${ }^{13}$, soil ${ }^{14}$ and wastewater ${ }^{15}$ environments. It still remains poorly understood whether specific environmental factors, such as the traits of the substrate, affect the relative fitness of strains engaged in MDOL, and thus govern the assembly of these communities.

Moreover, microorganisms often live in spatially structured environments, forming well-organized biofilms ${ }^{16,17}$. Diverse interactions among species within these biofilms drive to intricate spatiotemporal dynamics, which in turn, affect the assembly and 
productivity of such communities ${ }^{17-20}$. Particularly, communities engaged in MDOL commonly form specific patterns. For example, a number of studies have previously reported that anaerobic methanotrophic archaea at the surface of a deep seabed perform the first step of oxidation of methane, converting methane to hydrogen, before being transformed into bicarbonate by their bacterial partners $\mathrm{s}^{21-24}$. These studies also found that such communities self-organize into microcolonies with different morphologies, such as mixed-type $e^{21,25,26}$, mat-type ${ }^{21,25}$, as well as shell-type $e^{21,27,28}$. In these colonies, the average distances between cells from different populations, i.e., the levels of intermixing, vary considerably, which appears to be correlated with the taxa of the archaea and bacteria ${ }^{21}$. Another recent study investigating how a well-defined community engaged in MDOL spatially self-organized on surface, suggested that MDOL generally favored strong intermixing between the two strains involved, and the levels of intermixing are also correlate with the degree of wetness at the surface ${ }^{29}$. Therefore, the levels of intermixing of different strains within MDOL communities appear to rely on abiotic conditions. Therefore, insights into the factors affecting the levels of intermixing among different strains is critical for a more detailed understanding of the rules governing community assembly in heterogeneous environments.

Here, we set out to investigate how MDOL drives assembly and spatial organization of microbial communities by combining mathematical modelling and experimental research using a synthetic microbial community. More specifically, we focused on the effect of substrate traits, that is, its initial concentration and its toxicity. 


\section{Results}

\section{The hypotheses of this study}

We first proposed a hypothesis on how substrate concentration and its toxicity govern the assembly of microbial community engaged in MDOL, which is based on the feature of resource allocation among different members executing organic compounds degradation via MDOL. In these cases, the direct carbon sources, such as small organic acids or coenzyme A, to support bacterial growth, but these available nutrients are normally present as the final product of a pathway (Supplementary Figure 1). Therefore, the population performing the last steps can preferentially acquire and privatize these nutrients (which we henceforth call product privatization), thus acquiring the greater benefit, while other members have to collect nutrients released from this population (Figure 1A). We proposed a hypothesis based on this phenomenon: in an organic compound degradation community engaged in MDOL, the population executing the last steps of the pathway will dominate the community due to its privatization of the limited resources (we called this benefit private benefit below). This selfish population is analogous to a human worker responsible for the final step of an assembly line, who pockets the final product and rarely share profits with other workers. Thus, we named this final strain at the end of a MDOL-enabled pathway the 'Embezzler'. Following this hypothesis, we also derived two corollaries. Firstly, increasing concentration of the substrate improves the flux of the pathway, whereby more end product is released from the 'Embezzler' strain, in turn facilitating the growth of the other population (Figure 1B). Secondly, the presence of an initial substrate with a biotoxicity, which is frequently 
observed during organic compound degradation, favors the population performing the first step, because it is capable of detoxifying the initial substrate by transforming the substrate to the intermediates (Figure 1C). In summary, in presence of the resource privatization by the 'Embezzler' population, concentration and toxicity of the substrate can be two key factors that govern the assembly of organic compound degradation community engaged in MDOL. The goal of this study is to test this prediction.

\section{Individual-based modelling of spatial organization of MDOL community.}

To test our hypothesis about Embezzler behavior, as well as the effects of substrate concentration and toxicity, we simulated the assembly and spatial organization of MDOL community by constructing an individual-based model. As summarized in Supplementary Figure 2, we considered the degradation of an organic substrate (S) into an intermediate metabolite (I) by one enzyme (E1), before being degraded to the final product $(\mathrm{P})$ by a second enzyme (E2). We assumed that two strains carry out this pathway via MDOL, where the first strain only expresses E1, and the second only expresses E2. Initially, only S was supplied in the environment and evenly distributed across the entire habitat. Importantly, based on our hypothesis of how organic compounds are degraded in community engaged in MDOL, we assumed that, $\mathrm{P}$, which was synthesized by the second strain, was the sole available resource for the growth of both strains, while neither S nor I can be directly used for growth. Therefore, the second strain possessed the advantage of preferentially acquiring the resource, while the first strain only obtained those growth-limiting resource that were secreted from the second strain. Therefore, the second strain behaved as an 'Embezzler'. Moreover, biotoxicity 
150 of the substrate was imposed by adding a hyperbolic toxic term ${ }^{30}$ to the growth function,

151 and the toxic strength was mediated by parameter $t$. As the first population detoxified the toxic substrate, we named it 'Detoxifier'. Details about the model construction are described in Supplementary Information S1.

We initiated each simulation by randomly scattering a 1:1 (200-cell) mixture of the two cell types in a circle of $200-\mu \mathrm{m}$ radius. When toxicity was not considered and substrate concentration was low (e.g., $t=0$ and $S=5 \mathrm{C}-\mathrm{mmol} / \mathrm{L}$ ), the model successfully reproduced our hypothesis of 'Embezzler' behavior. In this case, 'Embezzler' cells grew much faster than Detoxifier cells and occupied large majority of the space with a relative fraction of $97.74 \% \pm 0.04 \%$ (Figure $2 \mathrm{~A}$; Supplementary video 1 ).

We then tested the corollary 1 stating that substrate concentration mediates the assembly of the community, by performing simulations with eight different initial substrate concentration and without substrate toxicity. As shown in Figure 2, with the increase of substrate concentration, the relative fraction of Detoxifier population increased and gradually reached a steady frequency around 35\% (Figure 2A and B; Supplementary video 2). To test whether this shift occurred due to the increase concentration of the final product released by the 'Embezzler' cells (i.e., corollary 1, Figure 1B), we analyzed the intracellular and extracellular product concentration of the two populations. Our analysis showed that with the increase of substrate input, the fraction of final product released by the Embezzler population (that is, the ratio of extracellular to intracellular concentration) increased (Supplementary Figure $3 \mathrm{~A}$ and B), so the Detoxifier obtained more product from the environment, resulted in a higher 
intracellular product concentration, which gradually approached that of the Embezzler (Supplementary Figure $3 \mathrm{~A}$ and C). Importantly, even if the substrate input was elevated to high levels (For example, $S=40 \mathrm{C}-\mathrm{mmol} / \mathrm{L}$ ), the intracellular product concentration of the Detoxifier never exceeded that of the Embezzler (Supplementary Figure $3 \mathrm{~A}$ and C). As a result, Embezzler cells maintained their growth advantage from privatizing end product. Together, these results showed that substrate concentration governs the assembly of community engaged in MDOL by affecting the amount of the final product exchange between the two populations. Nevertheless, in this scenario, the benefit from product privatization of the Embezzler strain cannot be completely eliminated by simply increasing substrate input.

We next included substrate toxicity into our model to test whether toxicity also affects community assembly (corollary 2). To this end, we performed simulations across three different toxic strength of the substrate as well as eight initial substrate concentration, and compared these results with previous results from the non-toxic scenario. Consistent with corollary 2, increasing substrate toxicity favors the 'Detoxifier' population (Figure 2A and B; Supplementary video 3). Our analyses further revealed that Detoxifier cells hold a lower intracellular concentration level of substrate (around $0.5 \mathrm{C}-\mathrm{mmol} / \mathrm{L}$ less) than Embezzler cells across all the conditions (Supplementary Figure 4), due to its conversion reaction, thus possessing a growth advantage over the detoxifier cells. Intriguingly, when the initial substrate concentration and substrate toxicity sufficiently high, Detoxifier population are able to dominate the community (that is, its relative frequency exceeded $50 \%$ of the community; see Figure 2B), 
suggesting that the benefit from product privatization of the Embezzler can be neutralized by higher substrate concentration and higher substrate toxicity.

To quantitively combine the effects of these two factors, we fit the simulation results by applying a simple polynomial function (named as PF1),

$$
D F=0.35+0.216 \sqrt[3]{t}-\frac{2.5}{S}
$$

where $\boldsymbol{D F}$ represents the fraction of the 'Detoxifier' population; $\boldsymbol{S}$ is the substrate concentration; $\boldsymbol{t}$ is the toxic strength of the substrate; 0.35 is the maximum frequency of Detoxifier populations when substrate is non-toxic $(t=0)$. The function fits well with the simulation data (Supplementary Figure $5 \mathrm{~A} ; \mathrm{R}^{2}=0.960$ ), and suggests a simple rule about how substrate concentration and toxicity govern the assembly of community involving MDOL: the frequency of the 'Detoxifier' increases nonlinearly with the increase of the initial substrate concentration, but holds a maximum value determined by substrate toxicity. Specifically, when $t$ exceeds approximately 0.335 , the maximum frequency of the 'Detoxifier' population can exceed 0.5 , i.e., the private benefit of the Embezzler is neutralized.

We then investigated whether MDOL affects the levels of intermixing of the populations in microbial communities. Therefore, we compared patterns developed in our basic model with those in a modified model considering that the two populations directly compete for a limited resource (See Supplementary Information S1 for the construction of this model). By analyzing the spatial assortment (See Methods and Supplementary Information S1 for the definition) of the cells in these patterns, we found that the presence of MDOL generally increases the intermixing level of the two strains 
in the community in most of the conditions (Figure 2C). However, the effect was correlated with the substrate concentration and toxicity. In 'Competition' scenarios, higher the substrate concentration is associated with increased intermixing level. As $\mathrm{mmol} / \mathrm{L})$ to $0.32 \pm 0.13(S=40 \mathrm{C}-\mathrm{mmol} / \mathrm{L})$, due to the thickened active cell layer during the range expansion as reported previously ${ }^{18}$. In contrast, in MDOL scenarios, both increasing substrate concentration and increasing the toxicity of the substrate reduced the intermixing level of the two strains in the formed spatial pattern (Figure 2C; Supplementary 6), as both strains formed large clusters under these conditions (Figure pathway is executed through MDOL in a community, both increasing substrate input and increasing the toxicity of the substrate favor the Detoxifier population, as well as reducing the levels of intermixing of the interacting populations in the formed pattern.

\section{Construction of a synthetic microbial community engaged in MDOL}

We next tested our model prediction by engineering a synthetic community composed of two P. stutzeri strains, both of which degrade an organic compound, salicylate, via metabolic division of labor (Figure 3). We engineered both strains from an ancestral strain, P. stutzeri AN0011, which is capable of autonomously degrading salicylate (Supplementary Figure 7). An operon located in the chromosome of AN0011 contains a series of the genes encoding the enzymes responsible for the conversion of salicylate (Figure 3A), which was previously engineered to be regulated by an IPTG-inducible $P_{t a c}$ promoter (unpublished data; Figure 3B). To construct the 'Detoxifier' strain $P$. 
stutzeri AN0010, we modified strain AN0011 by knocking out its nahH gene encoding catechol 2,3-dioxygenase, thus it was not capable of performing the second step. In addition, the $n a h G$ gene encoding salicylate 1-hydroxylase was deleted from AN0011 to generate the 'Embezzler' strain P. stutzeri AN0001 (Figure 3A and B). In addition, a strain lacking both $n a h G$ and $n a h H$ genes (Figure 3B) was constructed to serve as control strain that lacked the enzymes for both reaction steps, which we named

'Cheater'. Our analysis of enzymatic activity showed that Detoxifier only retained its ability to convert salicylate, while Embezzler was only able to metabolize the intermediate catechol (Supplementary Figure 7A). In liquid media, none of the Detoxifier, Embezzler, and Cheater strains grew autonomously using salicylate as the sole carbon source. However, Embezzler was capable of growing using catechol as the sole carbon source (Supplementary Figure 7B). When we co-cultured Detoxifier and Embezzler supplemented with a suitable range of concentration of salicylate, we found that the community grew at normal rates (Supplementary Figure 8A) and increasing the initial concentration of salicylate favored the 'Detoxifier' population in the final community, an observation that is in agreement with our model (Supplementary Figure 8B). As the intermediate catechol, cannot be directly used as the carbon source by both strains, the Embezzler strain possessed the preferential access to the final product, i.e., pyruvate and Acetyl-CoA (Figure 3A), which represented the direct carbon sources for the two strains in this community, consistent with our model assumption. In addition, salicylate was previously shown to be toxic for these $P$. stutzeri strains ${ }^{31}$. We quantitively measured its toxic strength (see Methods section measurement details). As 
shown in Figure 3C, the value of its toxic strength $(\boldsymbol{t})$ was $0.62 \mathrm{~L}^{-1} \mathrm{C}-\mathrm{mmol}$. Together, these results suggested that our synthetic community is suitable to experimentally test our hypotheses. For simplicity, we henceforth refer to our community as 'SMC-mdol'.

\section{Substrate concentration and toxicity govern the assembly and spatial organization}

\section{of the SMC-mdol}

To experimentally test whether substrate concentration and toxicity affect the assembly and spatial organization of community engaged in MDOL, we cultured the SMC-mdol community on an agarose surface (Figure 4A, second row) to which salicylate was added at different concentrations. To perform the corresponding mathematical simulations, we included the experimental scenarios (for example, one-unit salicylate would be converted to two-unit final product, i.e., one pyruvate and one acetyl-CoA) to our original model (Figure 4A, first row; denoted as 'Model'; see Supplementary Information S1 sections for detail about the model modification). We analyzed the structures of the synthetic community in different conditions, which indicate that the relative frequency of the Detoxifier population increased with the improvement of initial substrate concentration. As shown in Figure 4B, the frequency of Detoxifier population varied from $11.2 \% \pm 1.7 \%$ to $87.9 \% \pm 6.4 \%$ as a function of substrate concentration, exhibiting the same trends as obtained from our simulations, indicating that substrate concentration governs the assembly of microbial community engaged in MDOL (Figure 4B). Importantly, when the substrate input reached high levels, the Detoxifier population dominated the community (i.e., its relative fraction over $50 \%$ ), suggesting that substrate toxicity considerably affect the assembly of the community. 
Next, we generated a new fit function (named as PF2) based on our modified model related to the experimental scenarios (Supplementary Figure $5 B ; R^{2}=0.986$ ) to make quantitive prediction about the assembly of the community. PF2 shows the same form as PF1, but values of the coefficients exhibited slightly differences, as follow

$$
D F=0.49+0.144 \sqrt[3]{t}-\frac{1.25}{S}
$$

We then applied the measured value of toxic strength of salicylate (0.62) to this function to quantitively predict the final frequency of the Detoxifier population. As shown in Figure 4B (purple dashed line), the predictive power (Adjusted $\mathrm{R}^{2}$ ) was 0.51 . This result suggested that the assembly of our synthetic community follow the simple rule we proposed before, governed by substrate concentration and toxicity.

To examine the effects of substrate concentration and toxicity on spatial organization of the community, we calculated the spatial assortment of the patterns formed by our community and observation is similar as the model prediction, in which higher substrate concentration shaped a pattern with lower intermixing level of the two strains (Figure 4C). Nevertheless, the detail morphologies of the colony patterns showed some different characteristics compared with the simulated results. Firstly, at the edge of the developed colony, small 'sectors' were observed (Figure 4A, first row), which is usually explained by 'active layer effect' since limited resources are supplied and diffused from outside of the colony ${ }^{18}$. However, in our model, the only growth-limiting resource, $\mathrm{P}$, is produced by Embezzler cells, thus is supplied from more inside place of the colony. Further to this simple assumption, resources in addition to the carbon source such as nitrogen or phosphorus, were also growth-limiting in the experimental culturing system, 
and were supplied from outside of the colony. To overcome this difference, we introduced another limiting resource into the basic model. This resource is initially evenly supplied in the habitats and equally essential for the growth of both populations (Supplementary Figure 9A). This modification faithfully reproduced the 'sector' area in our further simulations (Figure 4A, denoted as 'Model-sectors'; Supplementary Figure 9B; Supplementary video 4). In addition, we generated an updated fit function (named as PF3) on the final frequency of the Detoxifier based on our new model (Supplementary Figure 5C; $\mathrm{R}^{2}=0.984$ ), namely

$$
D F=0.49+0.157 \sqrt[3]{t}-\frac{1.25}{S}
$$

PF3 only exhibited a slight shift of the coefficient compared with PF2 and predicted the assembly of the SMC-mdol with the predicting power (Adjusted $\mathrm{R}^{2}$ ) of 0.52 (Figure 4B, blue dashed line), indicating that this modification negligibly affects our rule on the assembly of community engaged in MDOL.

Secondly, the Detoxifier cells within the colony grew and formed bubble-like structures, while the Embezzler cells located around these bubbles (Figure 4A; Supplementary Figure 10A). Image analysis of these bubble structure clearly showed that the Detoxifier cells gathered together, exhibiting a 'bowl'-like geometrical morphology (Supplementary Figure 10B). Our analysis of the fluorescence intensity of the two cell types showed that Embezzler cells were mostly distributed around the bubble formed by the Detoxifier (Supplementary Figure 10C), suggesting that the interaction between the two populations required their cells spatially proximal to each other. Intriguingly, we found that the size of the 'bubble' structures formed by the Detoxifier population 
also changed with the increase of initial substrate concentration (Figure 4A, second row). We quantified this change by performing a segmentation imaging analysis of the 'bubble' area (Figure 5A), which indicated that with an increase in substrate concentration, while the number of the bubbles remained largely unchanged (Figure 5B), the average size of bubbles inside a colony significantly increased (Figure 5C), varying from $0.049 \pm 0.010 \mathrm{~mm}^{2}$ to $0.569 \pm 0.067 \mathrm{~mm}^{2}$. This suggests that Detoxifier cells tends to form larger cell clusters ('bubble' structures) with an increase in substrate concentration, resulting in lower intermixing levels of the two populations in the patterns (Figure 4C). Taken together, these results indicate that substrate concentration and toxicity govern the assembly and spatial organization of our synthetic community.

\section{Deficiency of the type IV pili of the both strains resulted in a spatial pattern more}

\section{similar as the simulated one}

Although the assembly of the SMC-mdol community resulted in a considerable match with our model prediction, the detail morphologies of the colonies differed only slightly (that is, the 'bubble' structures) with the simulated ones. Therefore, we assumed that, beside interplay via MDOL, other factors contributed to the formation of 'bubble' structures. It has been reported that cell appendages, such as type IV pilus and flagellum, play important roles in the Pseudomonas biofilm formation ${ }^{32-34}$. To investigate if these cell structures were involved in the 'bubble' formation, we knocked out the related genes. To deactivate the type IV pili, we simultaneously knocked out pilA and pilB genes of both AN0010 and AN0001. We also deleted $f \lg L$ and $f \lg K$ genes of the two strains to disrupt their flagella. Therefore, in addition to the SMC-mdol community, by 
co-culturing the pili deficient pair and flagella deficient pair, we constructed two additional synthetic communities named as 'SMC-mdol $\triangle p i l A B$ ' and 'SMCmdol $\triangle f l g L K$ '. Interestingly, while the SMC-mdol $\triangle f l g L K$ community still developed into patterns with 'bubble' structures inside, the community SMC-mdol $\triangle$ pilAB selforganized to a colony that better resembled the colony developed in our mathematical simulations (Figure 6A). We also cultured our three synthetic communities supplementing one of the final products, pyruvate, as the sole limited resource to simulate the 'competition' scenario, resulted in the segregated patterns as our model predictions (Figure 6A). Our analysis of spatial assortment revealed that MDOL between the two cell types generally increases their levels of intermixing, compared with those 'competition' scenarios (Figure 6B, unpaired, two-tailed, Student's t-test between the two scenarios of the three communities, $p<0.01$ ). In addition, deficiency of the type IV pili further drives cells of the two strains to translocate into closer proximity? (Figure $6 \mathrm{~B}, p<0.01$ ). Moreover, compared with the pattern formed by the SMC-mdol community and the SMC-mdol $\triangle f \lg L K$ community, colonies developed by the community SMC-mdol $\triangle$ pilAB produced more biomass, (Figure $6 \mathrm{C}, p<0.01$ ), suggesting that spatial proximity to each other facilitates the interaction between the two cell types, leading to better community-level productivity. In summary, these results strongly suggested that type IV pili are essential for the formation of the 'bubble' structures.

To further test our proposed rule, we cultured the SMC-mdol $\triangle$ pilAB community on agarose surface supplemented with different initial substrate concentration (Figure 4A, 
fourth row). We found the frequency of Detoxifier population increased from $29.4 \% \pm$ $2.8 \%$ to $79.2 \% \pm 2.6 \%$, which better agreed with the results obtained from the

372 simulations (Figure 4B). Notably, at high substrate levels, the Detoxifier population 373 also dominated the community (i.e., its relative fraction over $50 \%$ ). This result suggests 374 that substrate toxicity affected the assembly of the SMC-mdol $\triangle$ pilAB community in a 375 similar manner observed in the SMC-mdol community. In addition, both PF2 (Adjusted $376 \mathrm{R}^{2}$ equals 0.76 ) and PF3 (Adjusted $\mathrm{R}^{2}$ equals 0.78 ) predict the assembly of the SMC377 mdol $\triangle$ pilAB community better than that of the SMC-mdol community. These results 378 suggest that after excluding the effect of type IV pili, the assembly of synthetic community involving MDOL can be better predicted from the proposed rule. To

380 quantify the spatial patterns developed by the SMC-mdol $\triangle$ pilAB community, we 381 calculated the values of their spatial assortment, confirming the trend predicted by the 382 model simulations (Figure 4C and D). In summary, culture experiments with the SMCmdol $\triangle$ pilAB community again indicates that substrate concentration and toxicity 384 govern the assembly and spatial organization of microbial community involving 385 MDOL. 


\section{Discussion}

Here we show that substrate concentration and its toxicity drive the assembly of the microbial communities engaged in metabolic division of labor (MDOL), in which the population performing the first step is favored by both higher substrate concentration and its toxicity. We also found that the levels of intermixing between the interacting populations decreased with the increase of substrate concentration and toxicity, resulted in different spatial patterns.

Previous studies based on meta-omics approach revealed that MDOL were present in many microbial communities specialized in degrading organic compounds ${ }^{6,35}$. Inspired by these natural MDOL systems, numerous studies have recently explored strategies to divide metabolic roles across different populations in a consortium toward removal of organic pollutants ${ }^{36-41}$. While most of these studies only focused on the efficiency in pollutant degradation of these communities, a few of studies measured their community structures. For instance, one earlier study developed a dual-species community for biodegradation of the insecticide parathion ${ }^{42}$. In this consortium, an Escherichia coli strain SD2 was responsible for hydrolyzing parathion (Detoxifier), yielding two intermediates including p-nitrophenol, while another Pseudomonas putida strain KT2440 was responsible for metabolizing p-nitrophenol (Embezzler). That study found that the strain KT2440 that largely dominated the biofilm formed by this community, which is squarely in agreement with our basic prediction that the Embezzler strain is generally favored due to the 'private benefit'. Another study investigated the interactions among five bacterial species in a cellulose-degrading community, which 
also found that the strains responsible for the last step of the cellulose degradation constituted more than 60 percent of the total population ${ }^{43}$. Moreover, one recent study reported that a bacterial consortium composed of Leucobacter sp. GP and Achromobacter denitrificans PR1 efficiently degrades an antibiotic, sulfamethoxazole, in which the strain GP is responsible for the initial metabolism of the sulfamethoxazole (Detoxifier), and the strain PR1 carries out the subsequent conversion (Embezzler) ${ }^{44}$. This study measured the structures of the community across a gradient of initial substrate concentrations, and found that the frequency of the GP is positively correlated with the initial sulfamethoxazole concentration. This observation largely agrees idea derived from our model and experiments that increasing concentration of the substrate favors the Detoxifier population. Therefore, the proposed rule in our study may be expanded to forecast the assembly of other communities engaged in MDOL.

Based on our individual-based modelling, we showed that substrate toxicity is vital to govern the assembly of communities engaged in MDOL. However, due to the difficulty of manipulating the toxicity of the substrate (salicylate) in vitro, we did not experimentally compare the impacts of different toxic strength on the community assembly. However, our model predicts that simply increasing the initial substrate concentration is unlikely to shape a community dominated by the Detoxifier population, while the presence of substrate toxicity renders the dominance of the 'Detoxifier' population in the community. Therefore, the truth that Detoxifier population was able to dominate the synthetic community within higher substrate input, and the measured biotoxicity of salicylate, strongly suggested that substrate toxicity should be included 
in the assembly of the synthetic microbial community. Consistent with this idea, the prediction function (PF2 and PF3) involved in salicylate toxic strength fits the experiment results very well. To further examined this idea, we need to design a better synthetic community in which the toxicity of the substrate can be modulated. For example, several studies applied defined co-culture of multiple bacterial species to degrades antibiotics ${ }^{44-46}$. In these systems, antibiotics are toxic to all strains involved, but their toxicity may be modulated by genetically modifying the expression of the genes encoding the related antibiotic resistance. Performing pattern formation assays using these well-defined systems should assist in more thoroughly investigating the effects of substrate toxicity on the assembly of community.

In the colonies developed by SMC-mdol, we observed 'bubble'-like structures formed by the 'Detoxifier' cells, which has not been reported before. We found that the formation of these structures is highly correlated with the function of type IV pili. One important cell behavior mediated by type IV pili is bacterial twitching motility ${ }^{47,48}$. Indeed, we failed to observe twitching motility in mutants lacking pilA and pilB genes (Supplementary Figure 11). On the basis of this finding, we hypothesize that the observed 'bubble'-like structures were derived from active surface movement the Pseudomonas strains. Due to the heterogenous mass distribution of substrate or intermediates in environments that are spatially structured, the self-gathering behavior of our Detoxifier strain may be related to the chemotactic motility mediated by twitching motility ${ }^{48,49}$. Nevertheless, since type IV pili were also reported to be involved in other aspects of bacterial biofilm formation, such as surface sensing ${ }^{50}$ and 
surface attachment ${ }^{51,52}$, which may also contribute to the 'bubble' formation observed

454 in our experiments. How these types of single-cell behavior shape the macroscopic

455 colony pattern requires more detailed investigation at the single-cell level.

456 The ability to predict how the communities assembled by a given set of strains 457 exhibiting modularized functions is fundamental to engineer stable and high-efficient 458 microbial systems for bioproduction or biodegradation. Our results demonstrate that, 459 for a given community engaged in MDOL, its assembly can be quantitatively predicted 460 from the traits of its substrate, suggesting that it is feasible to control and manage microbial communities through manipulation of environmental factors. 


\section{Methods}

\section{Individual-based modeling}

The model was based on gro platform (https: https://github.com/liaupm/GRO-LIA), a simulator designed by Gutiérrez and colleagues aiming to describe multicellular bacterial behavior ${ }^{53}$. custom codes were written in gro language to describe interaction between two populations who implemented substrate degradation via MDOL. Supplementary Figure 2 provides an overview of the mathematical logic of the model. Variables and Parameters are summarized in Supplementary Table 1 and Supplementary Table 2, respectively. Details of the model framework and simulation workflow are described in Supplementary Information S1. The source gro codes of all the simulations used in this study, as well as the Mathematica (version 12.0) codes used for data analyses are available at Github:

\section{https://github.com/RoyWang1991/MDOLcode/tree/master/MDOL-spatial.}

\section{Construction of the $P$. stutzeri strains}

All $P$. stutzeri strains were engineered from a naphthalene-degrading bacterial strain $P$. stutzeri $\mathrm{AN} 10^{54}$. P. stutzeri AN0011 is a derived strain that can degrade salicylate autonomously, and all the genes encoding the corresponding enzymes were located in an operon induced by IPTG (unpublish data). To generate $P$. stutzeri AN0010 and $P$. stutzeri AN0001, nahG or nahH gene of P. stutzeri AN0011 was knocked out, respectively. Strain P. stutzeri AN0000 was generated from knocking out $n a h H$ gene of P. stutzeri AN0001. To generate the pilus mutants or flagellum mutants, pilA and pilB genes, or $f \lg L$ and $f \lg K$ genes, were simultaneously removed from the host strains, 
respectively. The genetic manipulation was implemented by allele exchange using the suicide plasmid $\mathrm{pK} 18$ mobsacB $^{55,56}$. The constructed strains were validated by PCR and DNA sequencing. In addition, enzymic activity assays were performed to verify the construction of $P$. stutzeri AN0011, P. stutzeri AN0010, P. stutzeri AN0001, as well as P. stutzeri AN0000, following the methods reported before (ref for salicylate 1hydroxylase and ref for catechol 2,3-dioxygenase). Furthermore, agar-based 'stab' assays were performed to validate the pilus mutants (Supplementary Figure 11), while 'swimming' assays were performed to validate flagellum mutants (Supplementary Figure 12), both following the standard $\operatorname{protocol}^{57}$. To label the strains with fluorescence, $m$ Cherry or $e G F P$ was cloned into a constitutive vector, $\mathrm{pMMPc-Gm}{ }^{58}$, and delivered to the host cells via triparental filter mating ${ }^{56}$.

\section{Liquid cultivation of the $P$. stutzeri strains}

To prepare the inoculum, $P$. stutzeri strains were first grown at $30^{\circ} \mathrm{C}$ RB liquid medium (Yeast extract $10 \mathrm{~g} / \mathrm{L}$, beef extract $6 \mathrm{~g} / \mathrm{L}$, peptone $10 \mathrm{~g} / \mathrm{L}$, ammonium sulfate $5 \mathrm{~g} / \mathrm{L}$ ) by shaking at $220 \mathrm{rpm}$, supplemented with $50 \mu \mathrm{g} / \mathrm{mL}$ gentamicin. The cells were then washed by the minimum medium ${ }^{59}$ for twice to make an inoculum. For co-culture experiments, inocula of two strains the were concentrated to an Optical density (OD, measured at $600 \mathrm{~nm}$ ) of 5.0, and mixed at a 1:1 ratio, and then inoculated to a $25-\mathrm{mL}$ flask containing $5 \mathrm{~mL}$ new fresh minimum medium (starting OD: 0.05), supplemented with $2 \mathrm{mM}$ IPTG, $50 \mu \mathrm{g} / \mathrm{mL}$ gentamicin, as well as salicylate as the sole carbon source. For mono-culture experiments, conditions were identical except the fact that the inoculum of one single strain was inoculated. Culture liquid $(100 \mu \mathrm{L})$ was taken from 
system to measure OD to estimate the total biomass, as well as measure fluorescence intensity to estimate the growth of each population. The relative fraction was calculated by a method previously described ${ }^{60}$. Briefly, cultures of each populations were grown to mid-log phase at $30^{\circ} \mathrm{C}(\mathrm{OD}: \sim 0.3)$, diluted two-fold for eleven times, and the dilutions measured for their OD and fluorescence. Correlations between OD and fluorescence were then determined using the basic method defined in the LinerModelFit function of Mathematica software. Eventually, fluorescence values were transformed to the ODestimated biomass to assess the growth of each population, and relative fraction was then calculated. These measurements, as well as the related measurements described below, were performed using a microplate reader (Molecular Devices, Sunnyvale, America).

\section{Measurement of salicylate toxicity}


$g_{s}=g_{\max } \cdot\left(\frac{1}{1+t \cdot S}\right)-d$

, where $\boldsymbol{g}_{\max }$ is the growth rate in absence of salicylate; $\boldsymbol{d}$ is the one-dimensional

530 death rate. The resulting fitted Adjust correlation coefficient $\left(\mathrm{R}^{2}\right)$ was 0.965 and the

531 quantified toxic strength of salicylate was 0.62 (Figure 3C).

\section{Colony pattern formation assays}

Inoculation and cultivation. Minimum medium (1.5\% agarose, Takara, Dalian, China),

supplemented with $2 \mathrm{mM}$ IPTG, $50 \mu \mathrm{g} / \mathrm{mL}$ gentamicin, and supplying salicylate or pyruvate as sole carbon source, was used in these studies. To prepare the culture plate, five milliliters of this medium was poured in a Petri dish (60 $\mathrm{mm}$ in diameter) and left on the bench overnight before inoculation. Preparation of the inocula is performed by the same way as liquid cultivation section. Inocula of two strains were then concentrated to an OD of 1.0, and mixed at a 1:1 ratio. For each colony, $1 \mu 1$ of the inoculum was spotted on the prepared plate, after which it was allowed to dry for 10 min. After the inoculum dried on the plate, the plates were incubated at $30{ }^{\circ} \mathrm{C}$ for 120 h.

Microscopy imaging. Colony patterns were imaged under $5 \times$ objective using a Leica DM6000B fluorescence microscope (Leica Corporation, Wetzlar, Germany) equipped with a LED fluorescence illuminator (Leica Corporation). Images were sequentially recorded with a DFC360 FX camera (Leica Corporation) using a GFP filter cube for eGFP (exciter: 475/40; emitter: 525/50: beamsplitter: 495) and a TX2 filter cube for mCherry (exciter: 560/40; emitter: 645/75: beamsplitter: 595). Tile scan function of Leica LAS X acquisition software (Leica Microsystems) were applied to assemble the 
full view of a colony from multiple fields, and composite images were also created by

551 this software.

Confocal imaging. To investigate the 3D structure of the 'bubble'-like structure, typical bubble areas were imaged under 10× objective using an Andor Revolution XD laser confocal fluorescence microscope (Andor, Oxford, UK) associated with ANDOR Zyla sCOMS camera. Individual color channels were acquired using the FLIC and TxRed filters in addition to a bright-field channel. The images were assembled and virtualized in Image $\mathbf{J}$ software (version $1.53 \mathrm{c}$ ). In addition, 'Surface Plot' function of Image $\mathbf{J}$ software was applied to analyze the relative intensity of fluorescence across the bubble areas.

Imaging analysis. Wolfram Mathematica (version 12.4) was used to process and analyze images. For the quantification of spatial assortment of each colony, the assembled eGFP and mCherry images were separately exported as grayscale tiff files, and then resized into images with 1000 pixels wide (using ImageResize function) to reduce computation time. Then ColorQuantize function was used to give an approximation to the image by quantizing it to distinct colors, subsequently transform the images into binarized data using ImageData function. Values of spatial assortment were calculated followed the protocol previous reported ${ }^{61,62}$, using these binarized matrix data as the input. Assortment is defined as the Embezzler segregation within their local neighborhood measured relative to its global frequencies. The Embezzler segregation was calculated by 


$$
\operatorname{seg}\left(E_{i}\right)=\frac{1}{n_{e}} \sum_{j=1}^{n_{e}} g\left(E_{i}, E_{j}\right)
$$

where $\boldsymbol{n}_{\boldsymbol{e}}$ is the number of cells falling within the distance of 50 pixels; $\boldsymbol{g}\left(\boldsymbol{E}_{\boldsymbol{i}}, \boldsymbol{E}_{\boldsymbol{j}}\right)=$

belong to the same population. The spatial assortment was then calculated by

$$
\operatorname{Assor}=\frac{\frac{1}{N_{e}} \sum_{j=1}^{N_{e}} \operatorname{seg}\left(E_{i}\right)-\mathrm{F}_{e}}{1-\mathrm{F}_{e}}
$$

where $\boldsymbol{N}_{\boldsymbol{e}}$ is the total number of Embezzler cells in the colony; $\mathbf{F}_{\boldsymbol{e}}$ is the frequency of

Embezzler cells in the colony. This value is bounded between -1 and 1, while assortment

values of zero reflect a well-mixed colony. For the quantification of the bubble structure

of each colony, color channel related to the 'Detoxifier' was used. We first cropped the images to focus on the inside area containing 'bubbles'. Then the 'bubbles' were segmented by the watershed algorithm using the WatershedComponents function of the

Wolfram Mathematica, followed by obtained the statistic data of the number and size of these structures by using ComponentMeasurements function. The area size in pixels were finally transformed to be the real size (in $\mathrm{mm}^{2}$ ) by multiply the scale bar, and virtualized by Colorize function. All these calculations were performed in custom

Wolfram Mathematica scripts. The source codes used are available on Github: https://github.com/RoyWang1991/MDOLcode/tree/master/MDOL-spatial.

Measurements of biomass and relative fraction. To estimate the biomass and community composition of a colony, spot was collected by an inoculation loop from the plate after 120-h's culture, and bacterial cells were resuspended in $100 \mu \mathrm{L}$ minimum 
measured to estimate the total biomass, and fluorescence intensities of eGFP and mCherry were measured to estimate the growth of each population. Calculation of the relative fraction followed the same method described in the Liquid cultivation section.

\section{Statistical analyses}

Unless indicated otherwise, the number of replicates is eight for each simulation and six for each experiment. For comparative statistics, unpaired, two-tailed, Student's ttest was performed in Wolfram Mathematica (version 12.4). To fit the data to the proposed function, Nonlinearmodelfit function of the Wolfram Mathematica (version 12.4) were applied.

\section{Competing Interests}

The authors declare that they have no conflict of interest.

\section{Acknowledgments}

We wish to thank Dr. Min Lin (Chinese Academy of Agricultural Sciences, Beijing, P.R. China) for providing plasmid pK18mobsacB and pRK2013, used for genetic engineering in this work. We also thank Professor Ping Xu (Shanghai Jiao Tong University, Shanghai, P.R. China) for supplying plasmid pMMPc-Gm, used for fluorescence labeling in this study. We are grateful to Professor Martin Ackermann (ETH Zurich, Zurich, Switzerland), Dr. David Johnson (Eawag, Dübendorf, Switzerland) and Yinyin Ma (Eawag, Dübendorf, Switzerland) for constructive inputs on the design of this study. We also appreciate Professor Martín Gutiérrez (Universidad Politécnica de Madrid, Madrid, Spain) for his kindly guidance for the set-up of the gro platform for the individual-based simulations. This work was supported by National 


\section{References}

6181 Kreft, J. U., Griffin, B. M. \& Gonzalez-Cabaleiro, R. Evolutionary causes and consequences of 619 metabolic division of labour: why anaerobes do and aerobes don't. Curr Opin Biotechnol 62, 80-87, doi:10.1016/j.copbio.2019.08.008 (2020).

622

Tsoi, R. et al. Metabolic division of labor in microbial systems. Proc Natl Acad Sci U S A 115, 2526-2531, doi:10.1073/pnas.1716888115 (2018).

Harvey, E., Heys, J. \& Gedeon, T. Quantifying the effects of the division of labor in metabolic pathways. J Theor Biol 360, 222-242, doi:10.1016/j.jtbi.2014.07.011 (2014). Thommes, M., Wang, T., Zhao, Q., Paschalidis, I. C. \& Segre, D. Designing Metabolic Division of Labor in Microbial Communities. mSystems 4, doi:10.1128/mSystems.00263-18 (2019).

5 Seelke, R. The Prokaryotes: a handbook on the biology of bacteria. Choice: Current Reviews for Academic Libraries 45, 68-68 (2007). Dombrowski, N. et al. Reconstructing metabolic pathways of hydrocarbon-degrading bacteria from the Deepwater Horizon oil spill. Nat Microbiol 1, 16057, doi:10.1038/nmicrobiol.2016.57 (2016). West, S. A. \& Cooper, G. A. Division of labour in microorganisms: an evolutionary perspective. Nat Rev Microbiol 14, 716-723, doi:10.1038/nrmicro.2016.111 (2016). van Gestel, J., Vlamakis, H. \& Kolter, R. Division of Labor in Biofilms: the Ecology of Cell Differentiation. Microbiol Spectr 3, MB-0002-2014, doi:10.1128/microbiolspec.MB-00022014 (2015).

9 Lilja, E. E. \& Johnson, D. R. Segregating metabolic processes into different microbial cells accelerates the consumption of inhibitory substrates. Isme Journal 10, 1568-1578, doi:10.1038/ismej.2015.243 (2016).

Schink, B. \& Pfennig, N. Fermentation of Trihydroxybenzenes by Pelobacter-Acidigallici GenNov Sp-Nov a New Strictly Anaerobic, Non-Sporeforming Bacterium. Arch Microbiol 133, 195-201, doi:Doi 10.1007/Bf00415000 (1982).

Wang, C. Y., Huang, Y., Zhang, Z. T., Hao, H. \& Wang, H. Absence of the nahG-like gene caused the syntrophic interaction between Marinobacter and other microbes in PAH-degrading process. J Hazard Mater 384, doi:10.1016/j.jhazmat.2019.121387 (2020).

Billet, L., Devers, M., Rouard, N., Martin-Laurent, F. \& Spor, A. Labour sharing promotes coexistence in atrazine degrading bacterial communities. Sci Rep 9, 18363, doi:10.1038/s41598019-54978-2 (2019).

Koudryashova, Y., Chizhova, T., Tishchenko, P. \& Hayakawa, K. Seasonal Variability of Polycyclic Aromatic Hydrocarbons (PAHs) in a Coastal Marine Area in the Northwestern Region of the Sea of Japan/East Sea (Possiet Bay). Ocean Sci J 54, 635-655, doi:10.1007/s12601-019-0031-9 (2019).

14 Jiang, Y., Hu, X., Yves, U. J., Zhan, H. \& Wu, Y. Status, source and health risk assessment of 
polycyclic aromatic hydrocarbons in street dust of an industrial city, NW China. Ecotoxicol Environ Saf 106, 11-18, doi:10.1016/j.ecoenv.2014.04.031 (2014).

15 Ozaki, N., Takamura, Y., Kojima, K. \& Kindaichi, T. Loading and removal of PAHs in a wastewater treatment plant in a separated sewer system. Water Res 80, 337-345, doi:10.1016/j.watres.2015.05.002 (2015).

16 Hall-Stoodley, L., Costerton, J. W. \& Stoodley, P. Bacterial biofilms: from the natural environment to infectious diseases. Nat Rev Microbiol 2, 95-108, doi:10.1038/nrmicro821 (2004).

17 Nadell, C. D., Drescher, K. \& Foster, K. R. Spatial structure, cooperation and competition in biofilms. Nat Rev Microbiol 14, 589-600, doi:10.1038/nrmicro.2016.84 (2016).

18 Nadell, C. D., Foster, K. R. \& Xavier, J. B. Emergence of Spatial Structure in Cell Groups and the Evolution of Cooperation. Plos Computational Biology 6, doi:10.1371/journal.pcbi.1000716 (2010).

19 Frost, I. et al. Cooperation, competition and antibiotic resistance in bacterial colonies. Isme Journal 12, 1582-1593, doi:10.1038/s41396-018-0090-4 (2018).

20 Momeni, B., Waite, A. J. \& Shou, W. Spatial self-organization favors heterotypic cooperation over cheating. Elife 2, e00960, doi:10.7554/eLife.00960 (2013).

21 Knittel, K. \& Boetius, A. Anaerobic oxidation of methane: progress with an unknown process. Annu Rev Microbiol 63, 311-334, doi:10.1146/annurev.micro.61.080706.093130 (2009).

22 Boetius, A. et al. A marine microbial consortium apparently mediating anaerobic oxidation of methane. Nature 407, 623-626, doi:10.1038/35036572 (2000).

23 Raghoebarsing, A. A. et al. A microbial consortium couples anaerobic methane oxidation to denitrification. Nature 440, 918-921, doi:10.1038/nature04617 (2006).

24 Ettwig, K. F. et al. Archaea catalyze iron-dependent anaerobic oxidation of methane. Proc Natl Acad Sci U S A 113, 12792-12796, doi:10.1073/pnas.1609534113 (2016).

25 Knittel, K., Losekann, T., Boetius, A., Kort, R. \& Amann, R. Diversity and distribution of methanotrophic archaea at cold seeps. Appl Environ Microb 71, 467-479, doi:10.1128/Aem.71.1.467-479.2005 (2005).

26 Nauhaus, K., Albrecht, M., Elvert, M., Boetius, A. \& Widdel, F. In vitro cell growth of marine archaeal-bacterial consortia during anaerobic oxidation of methane with sulfate. Environ Microbiol 9, 187-196, doi:10.1111/j.1462-2920.2006.01127.x (2007).

27 Losekann, T. et al. Diversity and abundance of aerobic and anaerobic methane oxidizers at the Haakon Mosby mud volcano, Barents Sea. Appl Environ Microb 73, 3348-3362, doi:10.1128/Aem.00016-07 (2007).

28 Niemann, H. et al. Novel microbial communities of the Haakon Mosby mud volcano and their role as a methane sink. Nature 443, 854-858, doi:10.1038/nature05227 (2006).

29 Tecon, R. \& Or, D. Cooperation in carbon source degradation shapes spatial self-organization of microbial consortia on hydrated surfaces. Sci Rep-Uk 7, doi:10.1038/srep43726 (2017).

30 Quintas, C., Leyva, J. S., Sotoca, R., Loureiro-Dias, M. C. \& Peinado, J. M. A model of the specific growth rate inhibition by weak acids in yeasts based on energy requirements. Int $J$ Food Microbiol 100, 125-130, doi:10.1016/j.jifoodmicro.2004.10.009 (2005).

31 Lanfranconi, M. P. et al. Physiological role of NahW, the additional salicylate hydroxylase found in Pseudomonas stutzeri AN10. Fems Microbiol Lett 300, 265-272, doi:10.1111/j.15746968.2009.01787.x (2009). 
32 Klausen, M. et al. Biofilm formation by Pseudomonas aeruginosa wild type, flagella and type IV pili mutants. Mol Microbiol 48, 1511-1524, doi:10.1046/j.1365-2958.2003.03525.x (2003).

33 Barken, K. B. et al. Roles of type IV pili, flagellum-mediated motility and extracellular DNA in the formation of mature multicellular structures in Pseudomonas aeruginosa biofilms. Environ Microbiol 10, 2331-2343, doi:10.1111/j.1462-2920.2008.01658.x (2008).

34 O'Toole, G. A. \& Kolter, R. Flagellar and twitching motility are necessary for Pseudomonas aeruginosa biofilm development. Mol Microbiol 30, 295-304, doi:10.1046/j.13652958.1998.01062.x (1998).

$35 \mathrm{Yu}, \mathrm{K}$. et al. An integrated meta-omics approach reveals substrates involved in synergistic interactions in a bisphenol A (BPA)-degrading microbial community. Microbiome 7, 16, doi:10.1186/s40168-019-0634-5 (2019).

$36 \mathrm{Li}$, Z. et al. Anaerobic mineralization of 2,4,6-tribromophenol to $\mathrm{CO} 2$ by a synthetic microbial community comprising Clostridium, Dehalobacter, and Desulfatiglans. Bioresour Technol 176, 225-232, doi:10.1016/j.biortech.2014.10.097 (2015).

37 Che, S. \& Men, Y. Synthetic microbial consortia for biosynthesis and biodegradation: promises and challenges. J Ind Microbiol Biotechnol 46, 1343-1358, doi:10.1007/s10295-019-02211-4 (2019).

38 Hudcova, T., Halecky, M., Kozliak, E., Stiborova, M. \& Paca, J. Aerobic degradation of 2,4dinitrotoluene by individual bacterial strains and defined mixed population in submerged cultures. J Hazard Mater 192, 605-613, doi:10.1016/j.jhazmat.2011.05.061 (2011).

39 Yang, C. et al. Atrazine degradation by a simple consortium of Klebsiella sp. A1 and Comamonas sp. A2 in nitrogen enriched medium. Biodegradation 21, 97-105, doi:10.1007/s10532-009-9284-9 (2010).

40 Roell, G. W. et al. Engineering microbial consortia by division of labor. Microb Cell Fact 18, 35, doi:10.1186/s12934-019-1083-3 (2019).

41 Jia, X. Q., He, Y., Jiang, D. W., Liu, C. \& Lu, W. Y. Construction and analysis of an engineered Escherichia coli-Pseudomonas aeruginosa co-culture consortium for phenanthrene bioremoval. Biochem Eng J 148, 214-223, doi:10.1016/j.bej.2019.05.010 (2019).

42 Gilbert, E. S., Walker, A. W. \& Keasling, J. D. A constructed microbial consortium for biodegradation of the organophosphorus insecticide parathion. Appl Microbiol Biot 61, 77-81, doi:10.1007/s00253-002-1203-5 (2003).

43 Kato, S., Haruta, S., Cui, Z. J., Ishii, M. \& Igarashi, Y. Stable coexistence of five bacterial strains as a cellulose-degrading community. Appl Environ Microb 71, 7099-7106, doi:10.1128/Aem.71.11.7099-7106.2005 (2005).

44 Reis, A. C. et al. Biodegradation of sulfamethoxazole by a bacterial consortium of Achromobacter denitrificans PR1 and Leucobacter sp GP. Appl Microbiol Biot 102, 1029910314, doi:10.1007/s00253-018-9411-9 (2018).

45 Liu, C. X., Xu, Q. M., Yu, S. C., Cheng, J. S. \& Yuan, Y. J. Bio-removal of tetracycline antibiotics under the consortium with probiotics Bacillus clausii $\mathrm{T}$ and Bacillus amyloliquefaciens producing biosurfactants. Sci Total Environ 710, doi:10.1016/j.scitotenv.2019.136329 (2020).

46 Kong, X. X. et al. The biodegradation of cefuroxime, cefotaxime and cefpirome by the synthetic consortium with probiotic Bacillus clausii and investigation of their potential biodegradation pathways. Sci Total Environ 651, 271-280, doi:10.1016/j.scitotenv.2018.09.187 (2019). 
Barken, K. B. et al. Roles of type IV pili, flagellum-mediated motility and extracellular DNA in the formation of mature multicellular structures in Pseudomonas aeruginosa biofilms. Environmental Microbiology 10, 2331-2343, doi:10.1111/j.1462-2920.2008.01658.x (2008).

48 Oliveira, N. M., Foster, K. R. \& Durham, W. M. Single-cell twitching chemotaxis in developing biofilms. Proc Natl Acad Sci U S A 113, 6532-6537, doi:10.1073/pnas.1600760113 (2016).

49 Miller, R. M. et al. Pseudomonas aeruginosa twitching motility-mediated chemotaxis towards phospholipids and fatty acids: Specificity and metabolic requirements. J Bacteriol 190, 40384049, doi:10.1128/Jb.00129-08 (2008).

50 Chang, C. Y. Surface Sensing for Biofilm Formation in Pseudomonas aeruginosa. Frontiers in Microbiology 8, doi:10.3389/fmicb.2017.02671 (2018).

51 Beaussart, A. et al. Nanoscale Adhesion Forces of Pseudomonas aeruginosa Type IV Pili. Acs Nano 8, 10723-10733, doi:10.1021/nn5044383 (2014).

52 Touhami, A., Jericho, M. H., Boyd, J. M. \& Beveridge, T. J. Nanoscale characterization and determination of adhesion forces of Pseudomonas aeruginosa Pili by using atomic force microscopy. J Bacteriol 188, 370-377, doi:10.1128/Jb.188.2.370-377.2006 (2006).

53 Gutierrez, M. et al. A New Improved and Extended Version of the Multicell Bacterial Simulator gro. ACS Synth Biol 6, 1496-1508, doi:10.1021/acssynbio.7b00003 (2017).

54 Brunet-Galmes, I. et al. Complete Genome Sequence of the Naphthalene-Degrading Bacterium Pseudomonas stutzeri AN10 (CCUG 29243). J Bacteriol 194, 6642-6643, doi:10.1128/Jb.01753-12 (2012).

55 Schafer, A. et al. Small mobilizable multi-purpose cloning vectors derived from the Escherichia coli plasmids pK18 and pK19: selection of defined deletions in the chromosome of Corynebacterium glutamicum. Gene 145, 69-73, doi:10.1016/0378-1119(94)90324-7 (1994).

56 Gao, C. et al. NAD-Independent L-Lactate Dehydrogenase Required for L-Lactate Utilization in Pseudomonas stutzeri A1501. J Bacteriol 197, 2239-2247, doi:10.1128/JB.00017-15 (2015). Filloux, A. \& Ramos, J. L. Preface. Pseudomonas methods and protocols. Methods Mol Biol 1149, v, doi:10.1007/978-1-4939-0473-0 (2014).

58 Xu, Y., Tao, F., Ma, C. \& Xu, P. New constitutive vectors: useful genetic engineering tools for biocatalysis. Appl Environ Microbiol 79, 2836-2840, doi:10.1128/AEM.03746-12 (2013).

59 Liang, J. L. et al. Regulation of alkane degradation pathway by a TetR family repressor via an autoregulation positive feedback mechanism in a Gram-positive Dietzia bacterium. Mol Microbiol 99, 338-359, doi:10.1111/mmi.13232 (2016).

60 Adamowicz, E. M., Flynn, J., Hunter, R. C. \& Harcombe, W. R. Cross-feeding modulates antibiotic tolerance in bacterial communities. Isme Journal 12, 2723-2735, doi:10.1038/s41396018-0212-z (2018).

61 Estrela, S. \& Brown, S. P. Metabolic and demographic feedbacks shape the emergent spatial structure and function of microbial communities. PLoS Comput Biol 9, e1003398, doi:10.1371/journal.pcbi.1003398 (2013).

62 Yanni, D., Marquez-Zacarias, P., Yunker, P. J. \& Ratcliff, W. C. Drivers of Spatial Structure in Social Microbial Communities. Curr Biol 29, R545-R550, doi:10.1016/j.cub.2019.03.068 (2019). 


\section{Figu re Legends}

Figure 1 Hypotheses for how substrate concentration and toxicity govern the assembly of community involving MDOL. In a community degrading an organic compound through metabolic division of labor (MDOL), final product was assumed to be the sole resource and was synthesized by the strain performing the last step. Therefore, this strain will obtain more nutrients (denoted as bigger 'smiling face'), while the other strain has to collect product released from this population (denoted as smaller 'smiling face'). Thus, the last population was named 'Embezzler'. However, increasing the concentration of the substrate (vertical axis) improves the flux of the pathway, leading to higher final product leakiness, favoring the growth of the first population. Moreover, introducing substrate biotoxicity (horizontal axis) also favors the first population, because it performs conversion of this toxic substrate (denoted as smaller sad face), resulted in lower intracellular substrate concentration than the Embezzler cells (denoted as bigger sad face). Thus, the first population was named as 'Detoxifier'.

Figure 2 Individual-based modelling suggests that substrate concentration and toxicity govern the assembly and spatial organization of community involving MDOL. (A) Representative colony patterns from the simulations in 'MDOL' scenarios across eight kinds of initial substrate concentrations and four different toxic strength, as well as the patterns developed when two populations simply compete for $\mathrm{P}$ ('Competition' scenarios). (B) Analysis of community composition of these colonies. Plot shows the relationship between substrate concentration and toxicity with the relative fraction of Detoxifier cells. (C) Analysis of spatial assortment (intermixing level) of these colonies. Here, an assortment value of 0 means cells of the two populations is randomly distributed (i.e., a well-mixed pattern), while a value of 1 means the distribution of the two populations is totally segregated. Plot shows the relationship between substrate concentration and toxicity with the spatial assortment of the colonies. As described in Methods 
section, the closer that the assortment value to zero means the corresponding pattern is more mixed. All the data were collected when the number of cells in the colony just reached 8100 . Eight replicated simulations were performed for each condition.

Figure 3 Design of the SMC-mdol. (A) Pathway of salicylate degradation in 'Superman' strain P. stutzeri AN0011, as well as partial pathways carried out by Detoxifier strain AN0010 and Embezzler strain AN0001. Skull marks that salicylate is toxic. (B) Diagram of operons responsible for the salicylate degradation, located in the chromosomes of AN0011, AN0010, AN0001 as well as a homologous mutant AN0000. (C) Determination of the toxic strength of the substrate salicylate. We measured the growth rate of AN0000 using pyruvate, one of the end products, as the carbon source, and supplying different concentration of salicylate. The growth rates $\left(\boldsymbol{g}_{\boldsymbol{s}}\right)$ were fitted with the corresponding salicylate concentration $(\boldsymbol{S})$, using a formula involving maximum growth rate $\left(\boldsymbol{g}_{\max }\right)$ and one-dimensional death rate $(\boldsymbol{d})$, showing a hyperbolic toxic effect (See Methods section for detailed description).

Figure 4 Substrate concentration and toxicity govern the assembly and spatial organization of the SMCmdol and SMC-mdol $\triangle$ pilAB. (A) Representative colony patterns from the pattern formation assays of SMC-mdol and SMC-mdol $\triangle p i l A B$, as well as the individual-based simulations with the modified model according to experimental parameters (denoted as 'Model') and the model with additional limited resource, L (denoted as 'Model-sectors'; see Supplementary information S1 for detail about the modifications of these models), across eight different initial substrate concentrations. (B) Analysis of community composition of the experimental formed colonies, as well as the model predicted colonies across eight kinds of initial substrate concentrations. The purple dashed line indicates the relative fraction of 'Detoxifier' calculated from the PF2, while the blue dashed line indicates the relative fraction of 'Detoxifier' calculated from the PF3. We use adjusted $\mathrm{R}^{2}$ to quantify the prediction power, where PF2 
shows a power of 0.51 for the assembly of SMC-mdol and 0.76 for that of SMC-mdol $\triangle$ pilAB, while PF3 shows a power of 0.52 for the assembly of SMC-mdol and 0.78 for that of SMC-mdol $\triangle$ pilAB. (CD) Analysis of spatial assortment experimental patterns (C) and the model predicted patterns (D) across eight different initial substrate concentrations. Note the absolute values of spatial assortment from the experiments and simulations are incomparable due to the difference between their scales (means experimental patterns containing more cells) and calculation methods (See Methods and Supplementary Information S1 sections for detail), but the trends of variation across the substrate concentrations are the same. For experiments, six replicates were performed for each condition, while for simulations, eight replicates were performed.

Figure 5 Substrate concentration governs the geometry of the 'bubble' structures inside the spatial patterns developed by SMC-mdol. (A) Workflow of the image analysis of the 'bubble' area. 'bubbles' formed by Detoxifier cells were segmented and analyzed to get its area size $\left(\mathrm{mm}^{2}\right)$. In the right graph, bubbles are color-coded based on their individual area size, with brighter colors indicating larger sizes. (B) Average number of the bubbles in the colony formed by SMC-mdol in different initial substrate concentration. (C) Average area size of the bubbles inside the colony formed by SMC-mdol in different initial substrate concentration. Images used here is same as Figure 4.

Figure 6 Type IV pili, but not flagella, are required for formation of the 'bubble' structures. (A) Images show that the colony patterning formed by the three synthetic communities in 'MDOL' scenario (supplying initial substrate salicylate as the sole carbon source) and 'Competition' scenario (supplying final product pyruvate as the sole carbon source). Typical morphology of the colony edges is zoomed in. Images were obtained after 120-h incubation. (B) Analysis of spatial assortment of the patterns formed in all conditions. As mentioned before, Assortment value closer to zero means the corresponding pattern 
850 is more mixed. (C) Analysis of community composition of the colony patterns formed in all conditions.

851 Six replicates were performed for each condition. Different lower-case letters in (B) or (C) indicate 852 significant difference among these conditions at 0.01 level (unpaired, two-tailed, Student's t-test). 


\section{Figures}

\section{Substrate Toxicity}

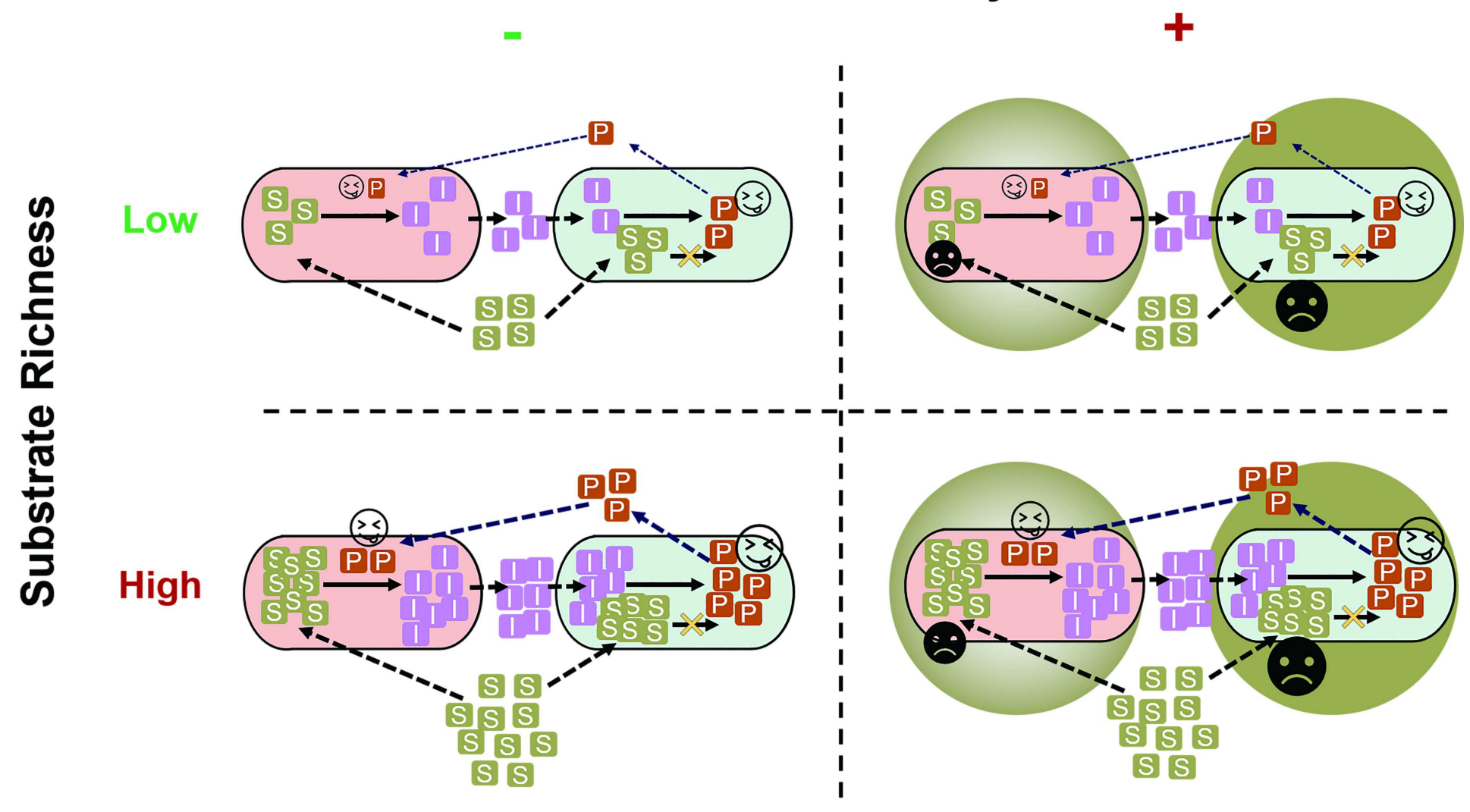

Figure 1

Hypotheses for how substrate concentration and toxicity govern the assembly of community involving MDOL. In a community degrading an organic compound through metabolic division of labor (MDOL), final product was assumed to be the sole resource and was synthesized by the strain performing the last step. Therefore, this strain will obtain more nutrients (denoted as bigger 'smiling face'), while the other strain has to collect product released from this population (denoted as smaller 'smiling face'). Thus, the last population was named 'Embezzler'. However, increasing the concentration of the substrate (vertical axis) improves the flux of the pathway, leading to higher final product leakiness, favoring the growth of the first population. Moreover, introducing substrate biotoxicity (horizontal axis) also favors the first population, because it performs conversion of this toxic substrate (denoted as smaller sad face), resulted in lower intracellular substrate concentration than the Embezzler cells (denoted as bigger sad face). Thus, the first population was named as 'Detoxifier'. 


\section{Substrate Toxicity}

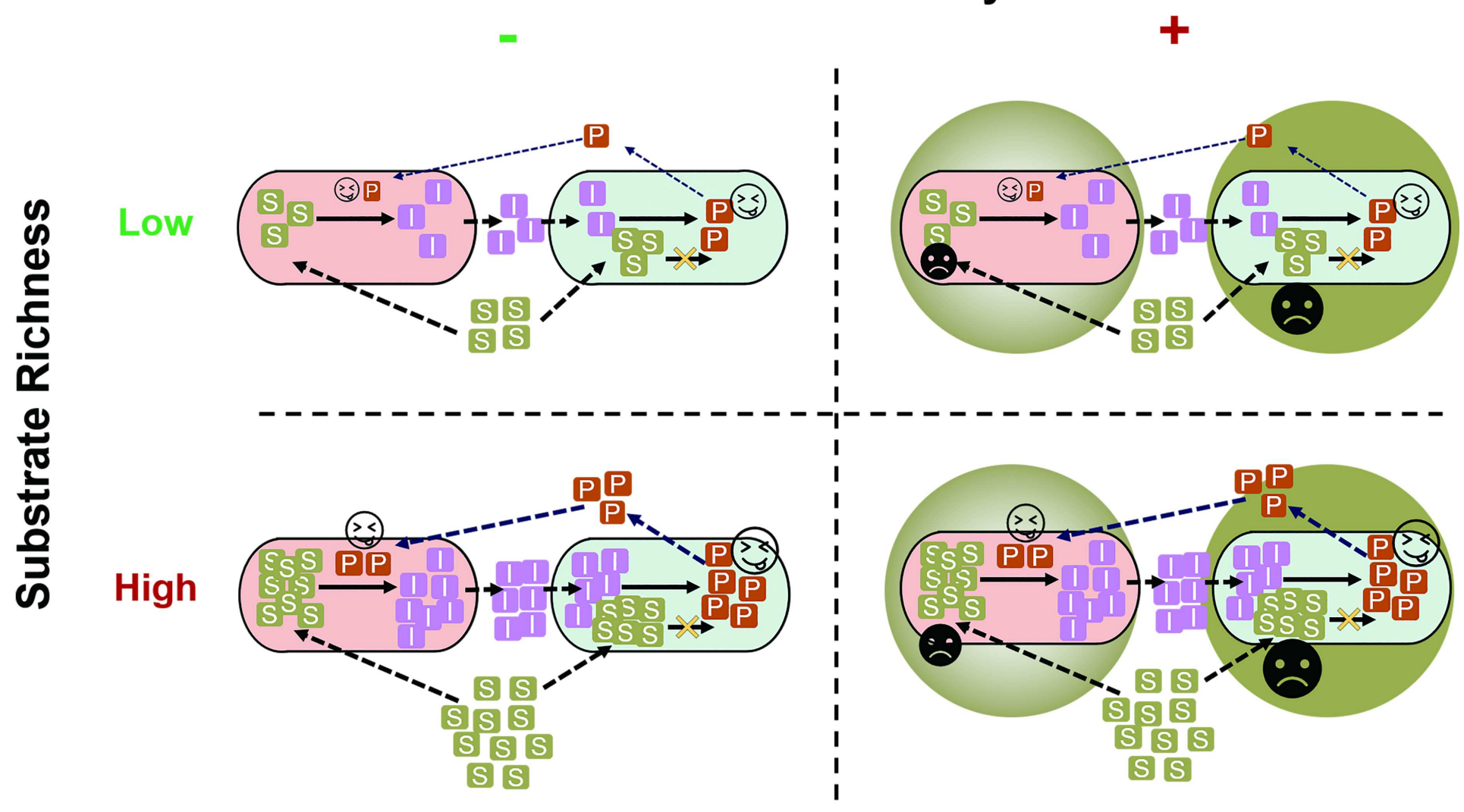

\section{Figure 1}

Hypotheses for how substrate concentration and toxicity govern the assembly of community involving MDOL. In a community degrading an organic compound through metabolic division of labor (MDOL), final product was assumed to be the sole resource and was synthesized by the strain performing the last step. Therefore, this strain will obtain more nutrients (denoted as bigger 'smiling face'), while the other strain has to collect product released from this population (denoted as smaller 'smiling face'). Thus, the last population was named 'Embezzler'. However, increasing the concentration of the substrate (vertical axis) improves the flux of the pathway, leading to higher final product leakiness, favoring the growth of the first population. Moreover, introducing substrate biotoxicity (horizontal axis) also favors the first population, because it performs conversion of this toxic substrate (denoted as smaller sad face), resulted in lower intracellular substrate concentration than the Embezzler cells (denoted as bigger sad face). Thus, the first population was named as 'Detoxifier'. 


\section{Substrate Toxicity}

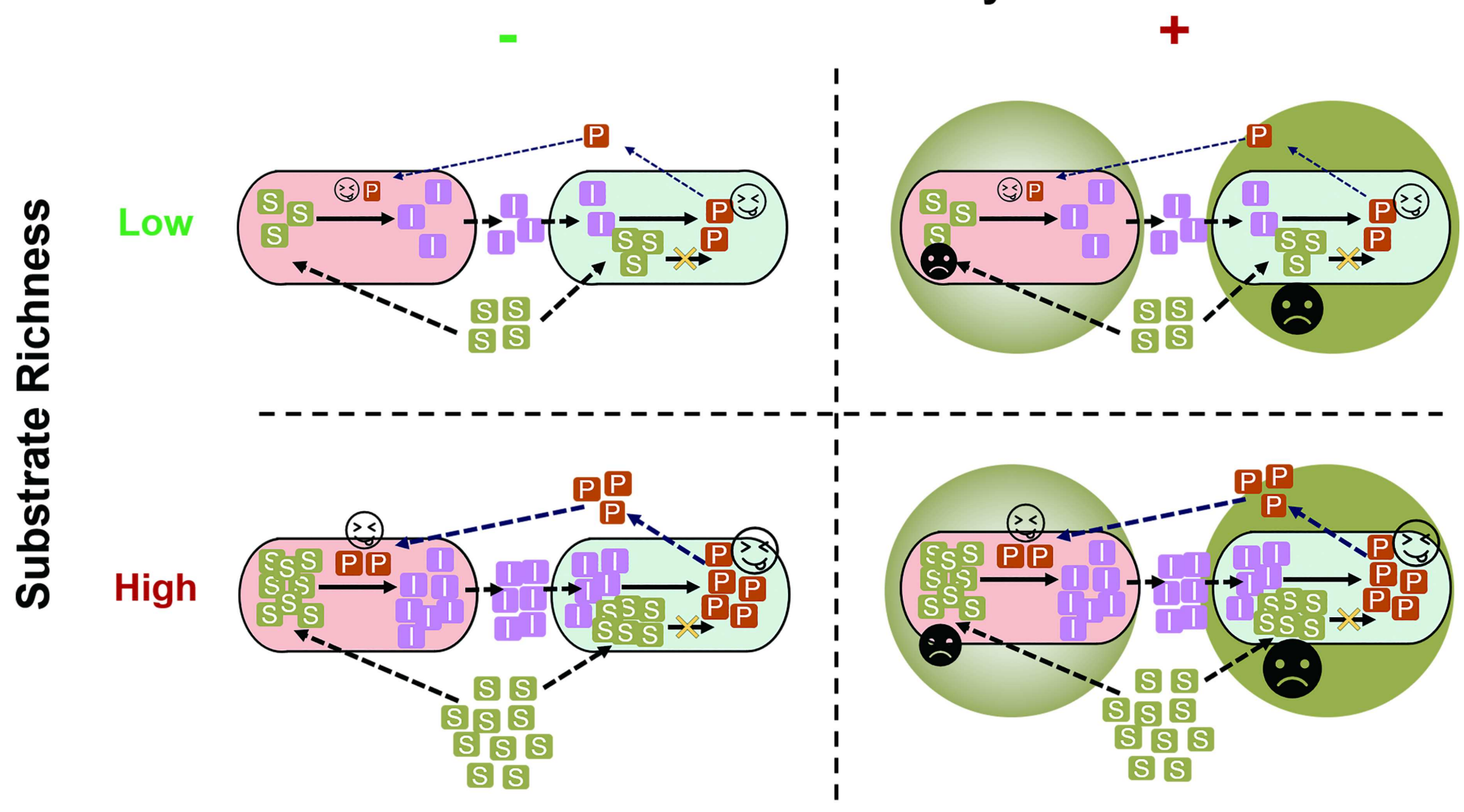

\section{Figure 1}

Hypotheses for how substrate concentration and toxicity govern the assembly of community involving MDOL. In a community degrading an organic compound through metabolic division of labor (MDOL), final product was assumed to be the sole resource and was synthesized by the strain performing the last step. Therefore, this strain will obtain more nutrients (denoted as bigger 'smiling face'), while the other strain has to collect product released from this population (denoted as smaller 'smiling face'). Thus, the last population was named 'Embezzler'. However, increasing the concentration of the substrate (vertical axis) improves the flux of the pathway, leading to higher final product leakiness, favoring the growth of the first population. Moreover, introducing substrate biotoxicity (horizontal axis) also favors the first population, because it performs conversion of this toxic substrate (denoted as smaller sad face), resulted in lower intracellular substrate concentration than the Embezzler cells (denoted as bigger sad face). Thus, the first population was named as 'Detoxifier'. 


\section{Substrate Toxicity}

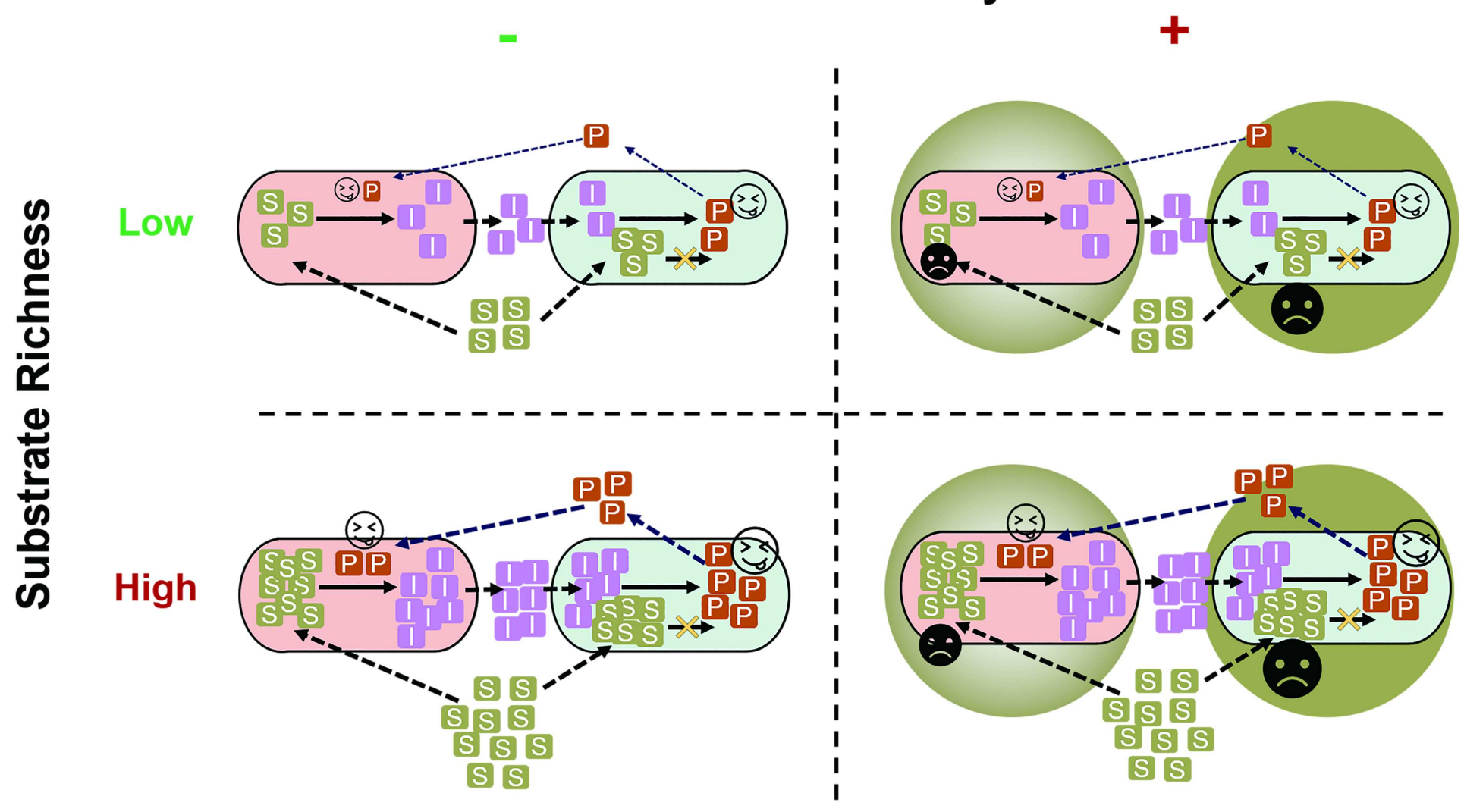

\section{Figure 1}

Hypotheses for how substrate concentration and toxicity govern the assembly of community involving MDOL. In a community degrading an organic compound through metabolic division of labor (MDOL), final product was assumed to be the sole resource and was synthesized by the strain performing the last step. Therefore, this strain will obtain more nutrients (denoted as bigger 'smiling face'), while the other strain has to collect product released from this population (denoted as smaller 'smiling face'). Thus, the last population was named 'Embezzler'. However, increasing the concentration of the substrate (vertical axis) improves the flux of the pathway, leading to higher final product leakiness, favoring the growth of the first population. Moreover, introducing substrate biotoxicity (horizontal axis) also favors the first population, because it performs conversion of this toxic substrate (denoted as smaller sad face), resulted in lower intracellular substrate concentration than the Embezzler cells (denoted as bigger sad face). Thus, the first population was named as 'Detoxifier'. 


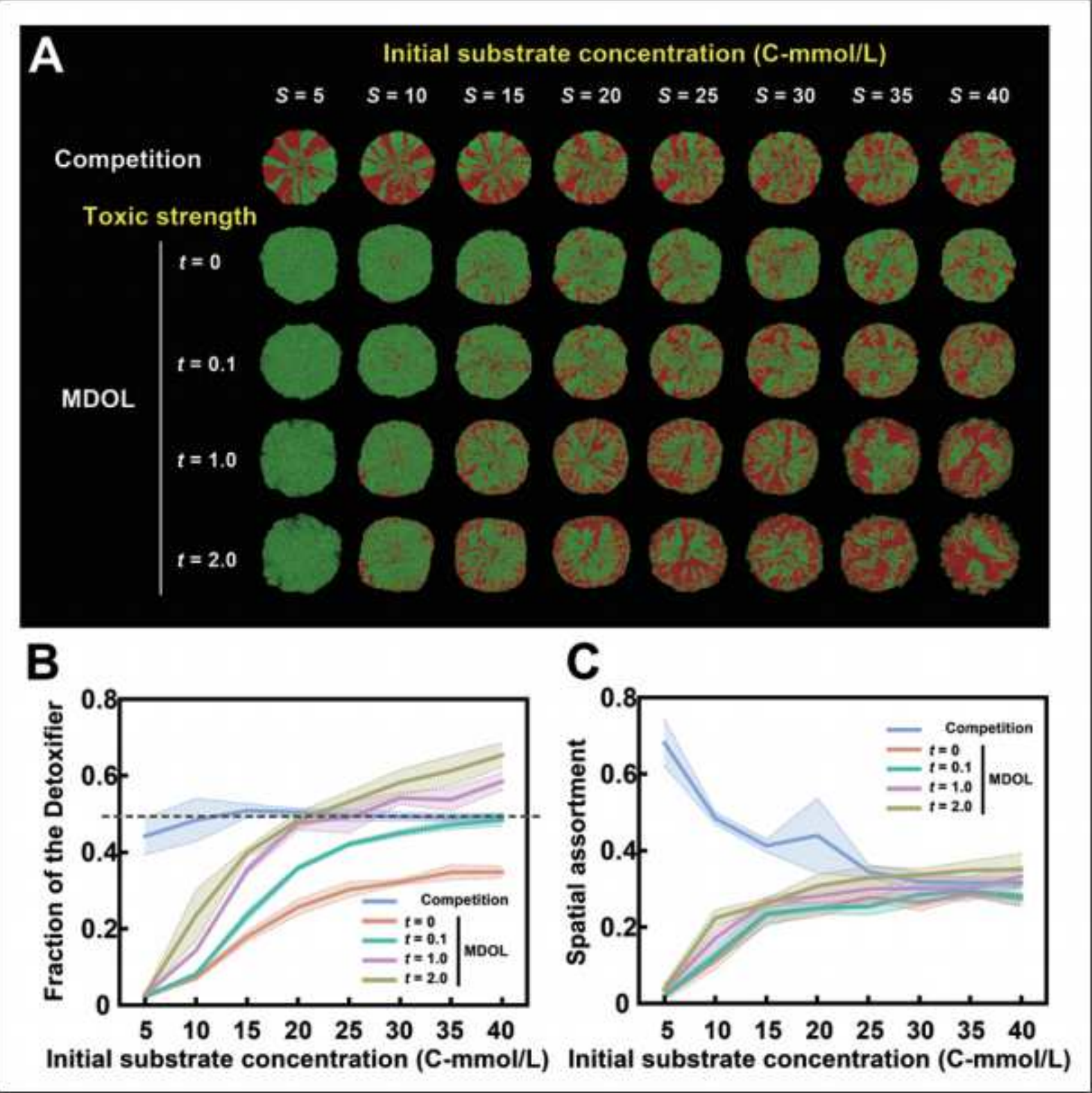

Figure 2

Individual-based modelling suggests that substrate concentration and toxicity govern the assembly and spatial organization of community involving MDOL. (A) Representative colony patterns from the simulations in 'MDOL' scenarios across eight kinds of initial substrate concentrations and four different toxic strength, as well as the patterns developed when two populations simply compete for $P$ ('Competition' scenarios). (B) Analysis of community composition of these colonies. Plot shows the relationship between substrate concentration and toxicity with the relative fraction of Detoxifier cells. (C) Analysis of spatial assortment (intermixing level) of these colonies. Here, an assortment value of 0 means cells of the two populations is randomly distributed (i.e., a well-mixed pattern), while a value of 1 means the distribution of the two populations is totally segregated. Plot shows the relationship between substrate concentration and toxicity with the spatial assortment of the colonies. As described in Methods section, the closer that the assortment value to zero means the corresponding pattern is more mixed. All 
the data were collected when the number of cells in the colony just reached 8100 . Eight replicated simulations were performed for each condition.

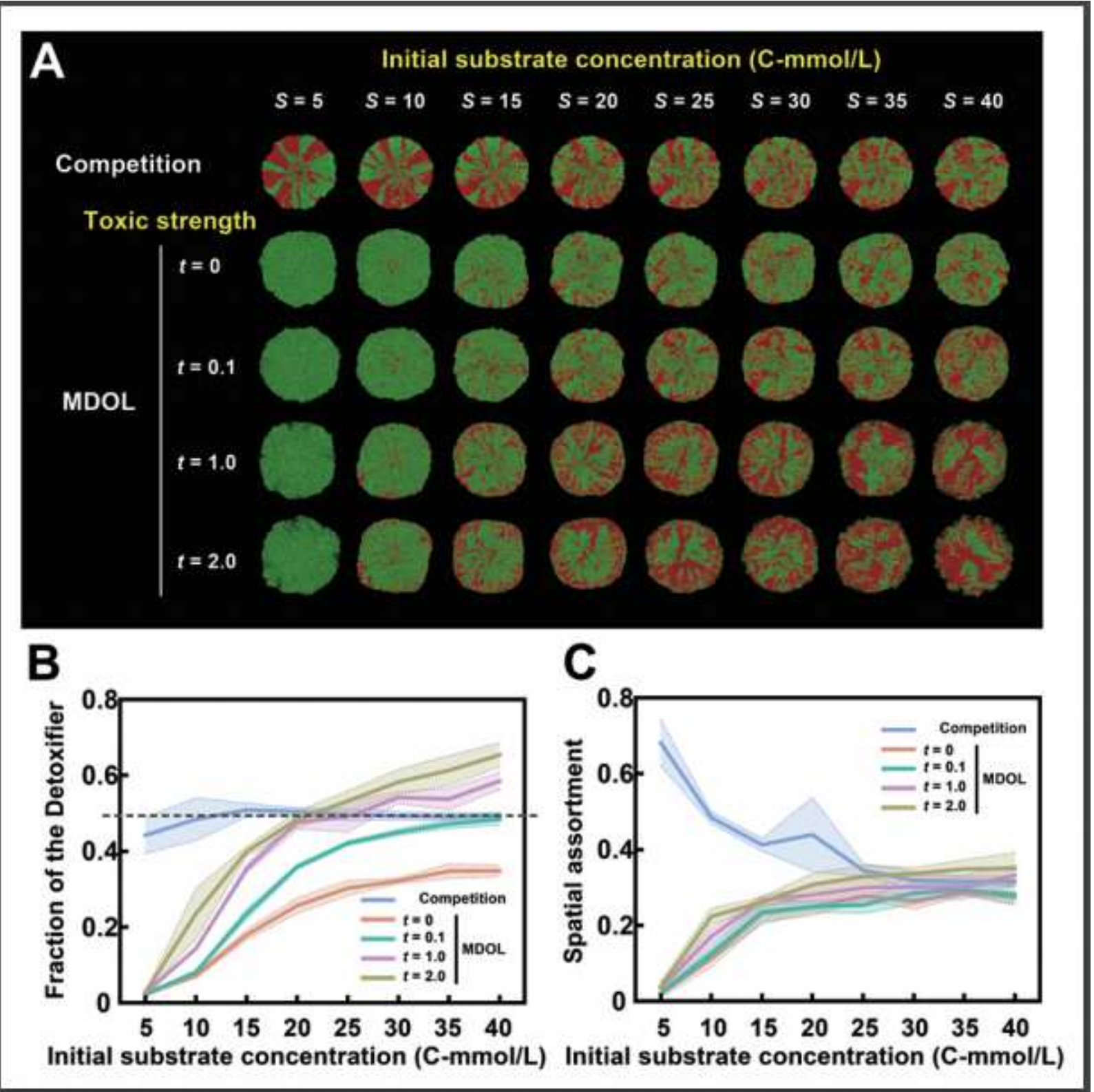

\section{Figure 2}

Individual-based modelling suggests that substrate concentration and toxicity govern the assembly and spatial organization of community involving MDOL. (A) Representative colony patterns from the simulations in 'MDOL' scenarios across eight kinds of initial substrate concentrations and four different toxic strength, as well as the patterns developed when two populations simply compete for $P$ ('Competition' scenarios). (B) Analysis of community composition of these colonies. Plot shows the relationship between substrate concentration and toxicity with the relative fraction of Detoxifier cells. (C) Analysis of spatial assortment (intermixing level) of these colonies. Here, an assortment value of 0 means cells of the two populations is randomly distributed (i.e., a well-mixed pattern), while a value of 1 means the distribution of the two populations is totally segregated. Plot shows the relationship between substrate concentration and toxicity with the spatial assortment of the colonies. As described in Methods 
section, the closer that the assortment value to zero means the corresponding pattern is more mixed. All the data were collected when the number of cells in the colony just reached 8100 . Eight replicated simulations were performed for each condition.

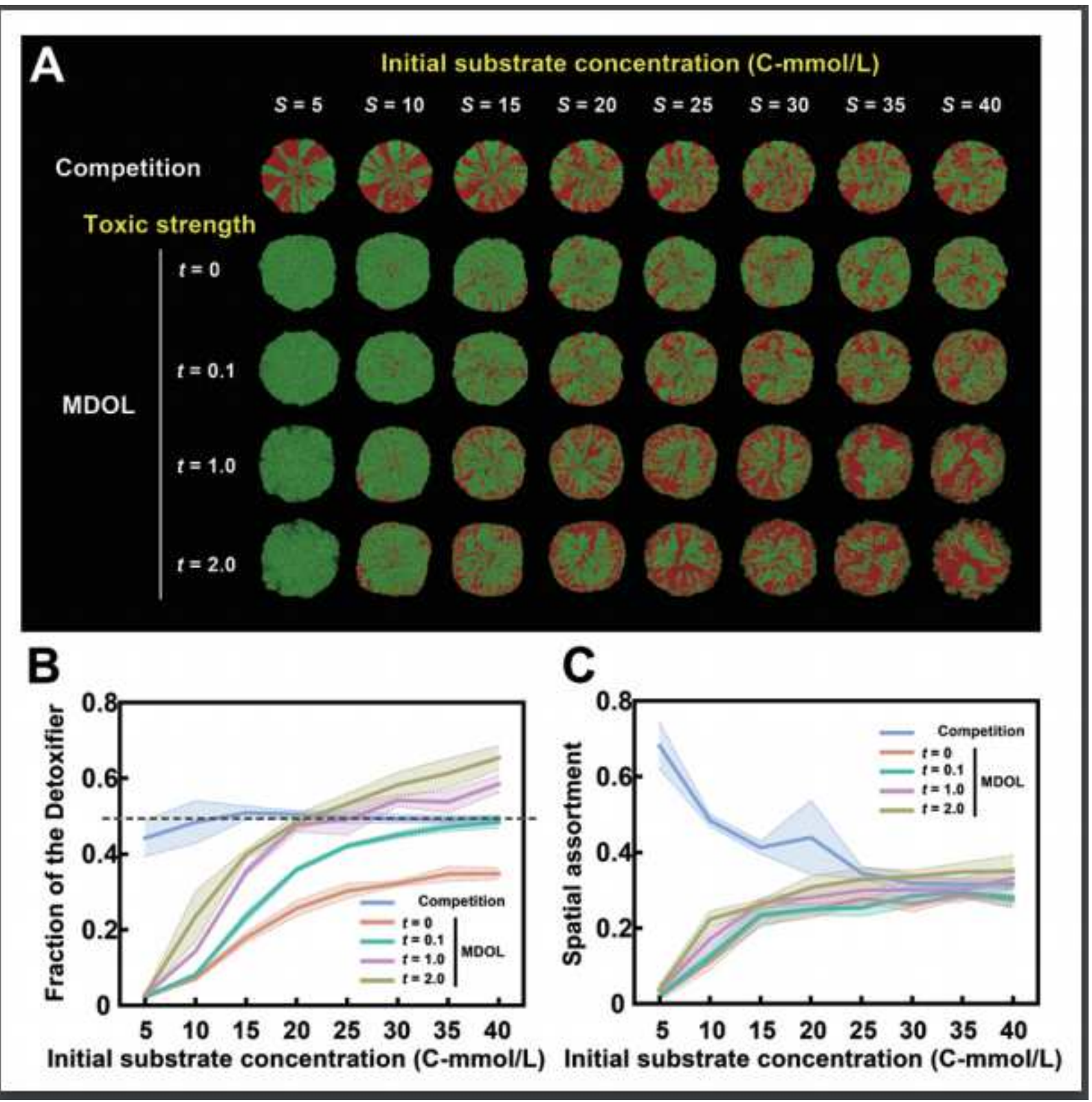

Figure 2

Individual-based modelling suggests that substrate concentration and toxicity govern the assembly and spatial organization of community involving MDOL. (A) Representative colony patterns from the simulations in 'MDOL' scenarios across eight kinds of initial substrate concentrations and four different toxic strength, as well as the patterns developed when two populations simply compete for $\mathrm{P}$ ('Competition' scenarios). (B) Analysis of community composition of these colonies. Plot shows the relationship between substrate concentration and toxicity with the relative fraction of Detoxifier cells. (C) Analysis of spatial assortment (intermixing level) of these colonies. Here, an assortment value of 0 means cells of the two populations is randomly distributed (i.e., a well-mixed pattern), while a value of 1 means the distribution of the two populations is totally segregated. Plot shows the relationship between 
substrate concentration and toxicity with the spatial assortment of the colonies. As described in Methods section, the closer that the assortment value to zero means the corresponding pattern is more mixed. All the data were collected when the number of cells in the colony just reached 8100 . Eight replicated simulations were performed for each condition.

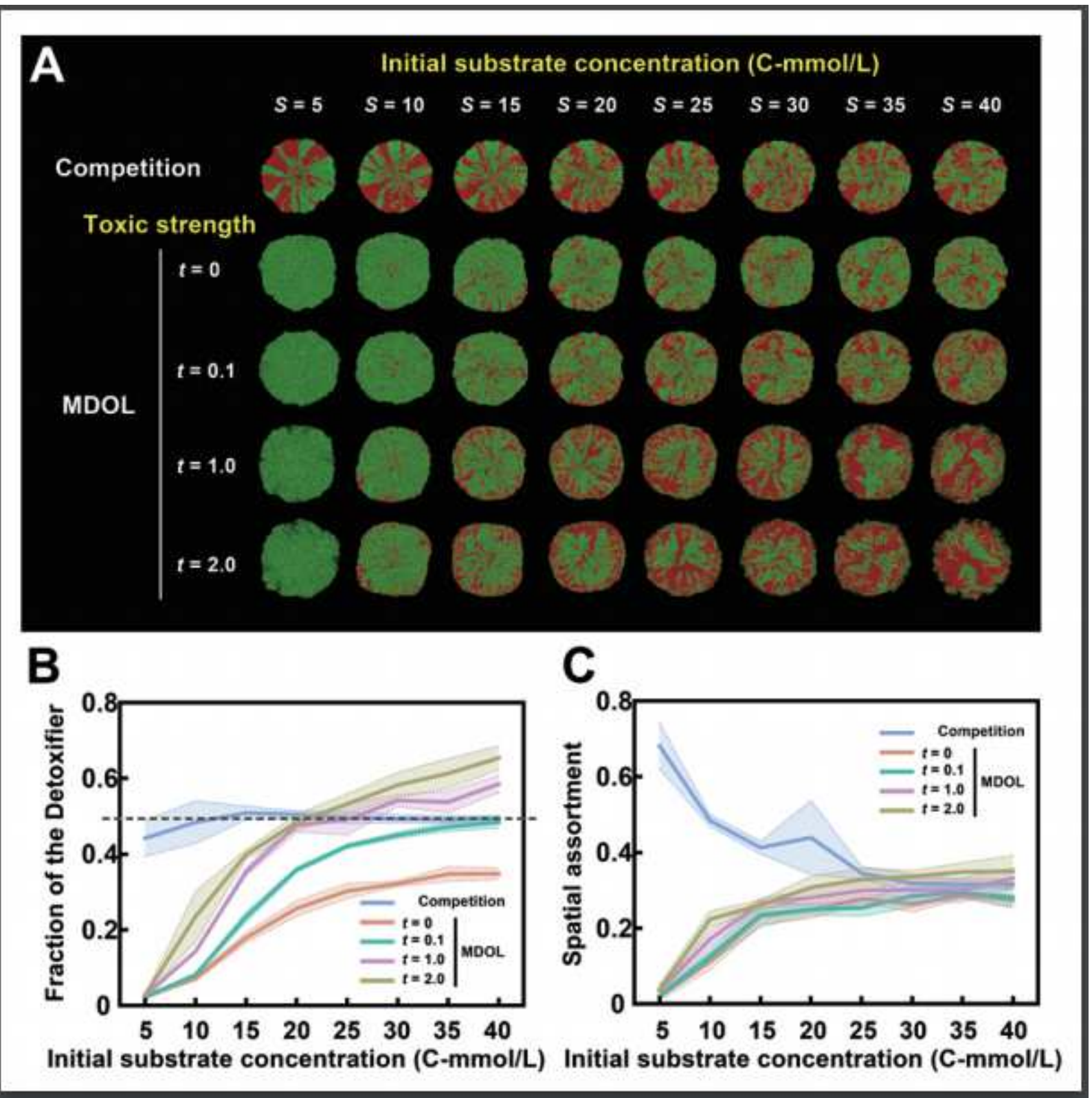

Figure 2

Individual-based modelling suggests that substrate concentration and toxicity govern the assembly and spatial organization of community involving MDOL. (A) Representative colony patterns from the simulations in 'MDOL' scenarios across eight kinds of initial substrate concentrations and four different toxic strength, as well as the patterns developed when two populations simply compete for $P$ ('Competition' scenarios). (B) Analysis of community composition of these colonies. Plot shows the relationship between substrate concentration and toxicity with the relative fraction of Detoxifier cells. (C) Analysis of spatial assortment (intermixing level) of these colonies. Here, an assortment value of 0 means cells of the two populations is randomly distributed (i.e., a well-mixed pattern), while a value of 1 
means the distribution of the two populations is totally segregated. Plot shows the relationship between substrate concentration and toxicity with the spatial assortment of the colonies. As described in Methods section, the closer that the assortment value to zero means the corresponding pattern is more mixed. All the data were collected when the number of cells in the colony just reached 8100 . Eight replicated simulations were performed for each condition.

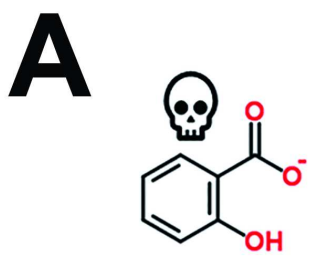

Salicylate
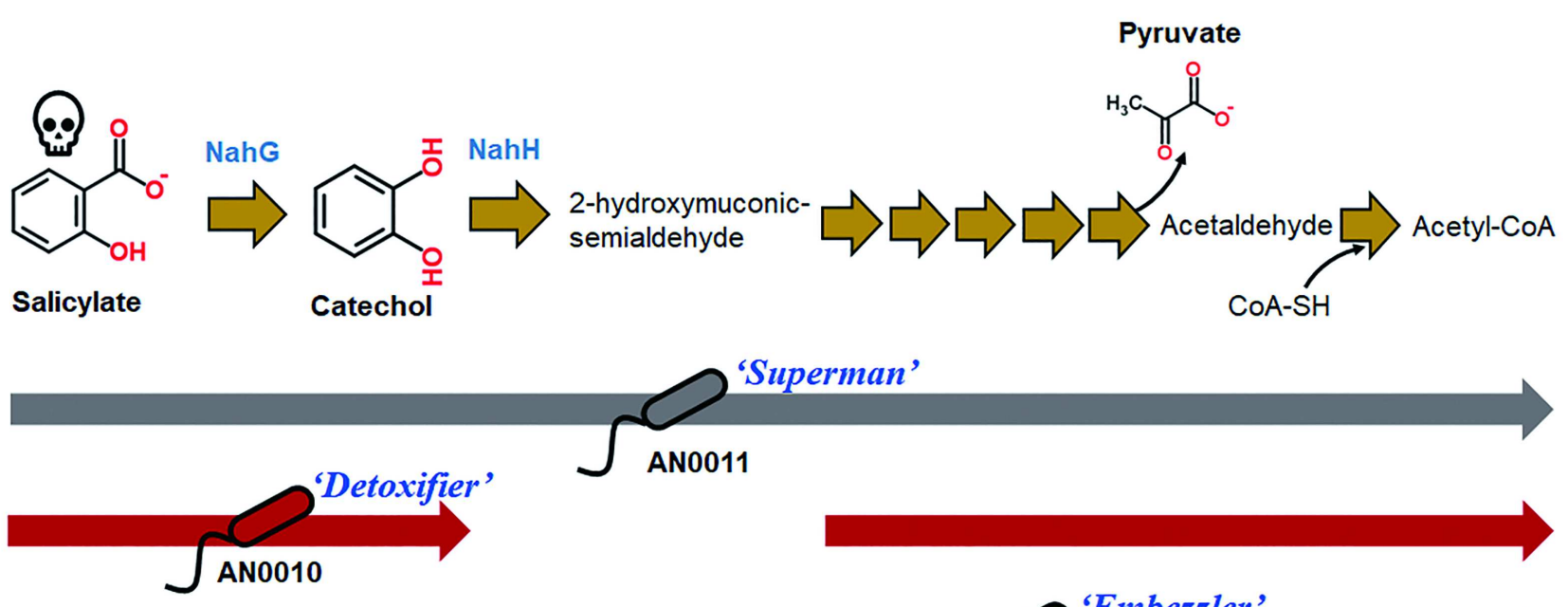

\section{'Superman'}

AN0011

'Embezzler'
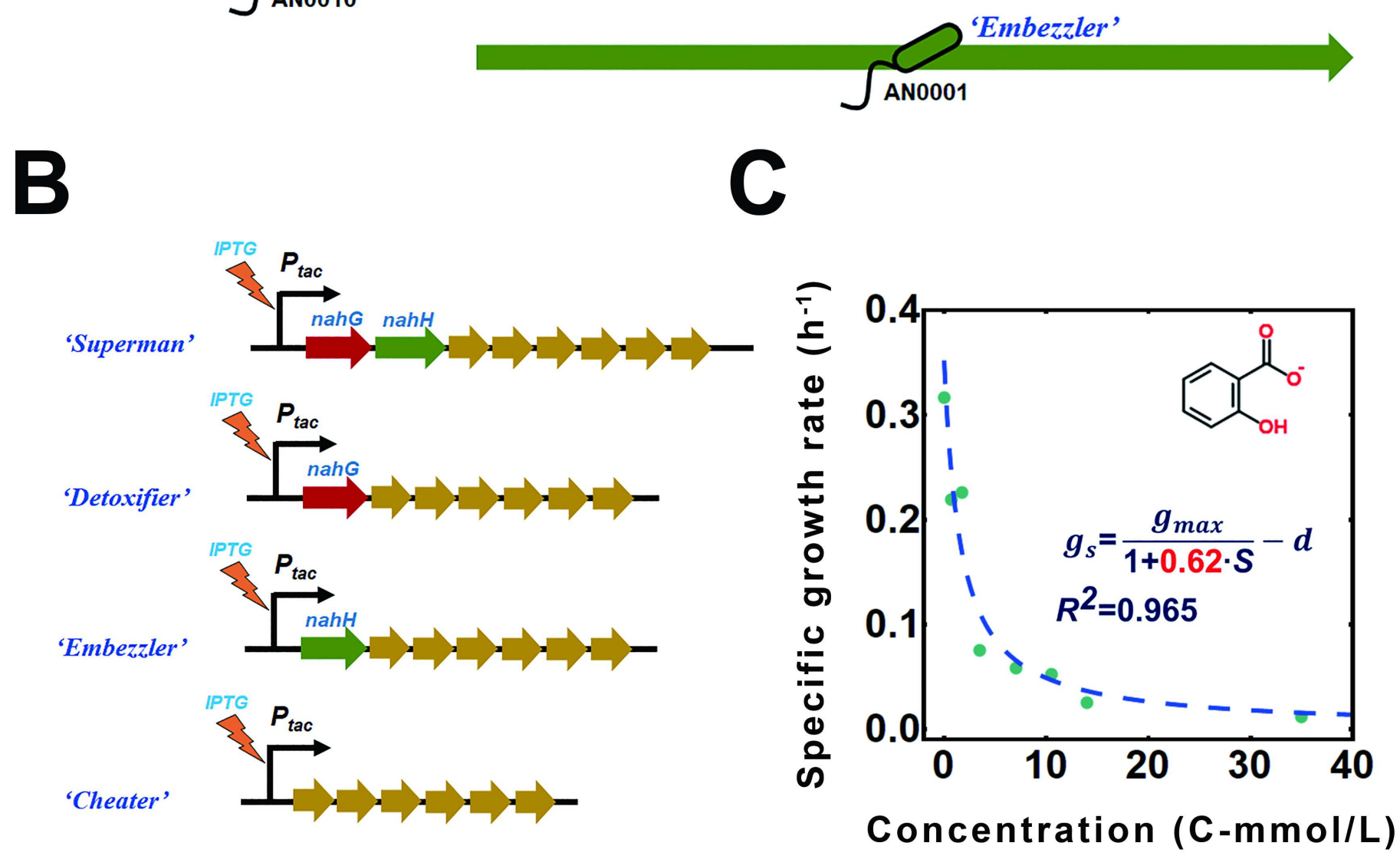

Figure 3

Design of the SMC-mdol. (A) Pathway of salicylate degradation in 'Superman' strain P. stutzeri AN0011, as well as partial pathways carried out by Detoxifier strain AN0010 and Embezzler strain AN0001. Skull marks that salicylate is toxic. (B) Diagram of operons responsible for the salicylate degradation, located in the chromosomes of AN0011, AN0010, AN0001 as well as a homologous mutant AN0000. (C) 
Determination of the toxic strength of the substrate salicylate. We measured the growth rate of AN0000 using pyruvate, one of the end products, as the carbon source, and supplying different concentration of salicylate. The growth rates (g_s) were fitted with the corresponding salicylate concentration (S), using a formula involving maximum growth rate (g_max) and one-dimensional death rate (d), showing a hyperbolic toxic effect (See Methods section for detailed description).

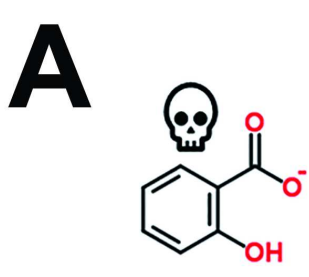

Salicylate
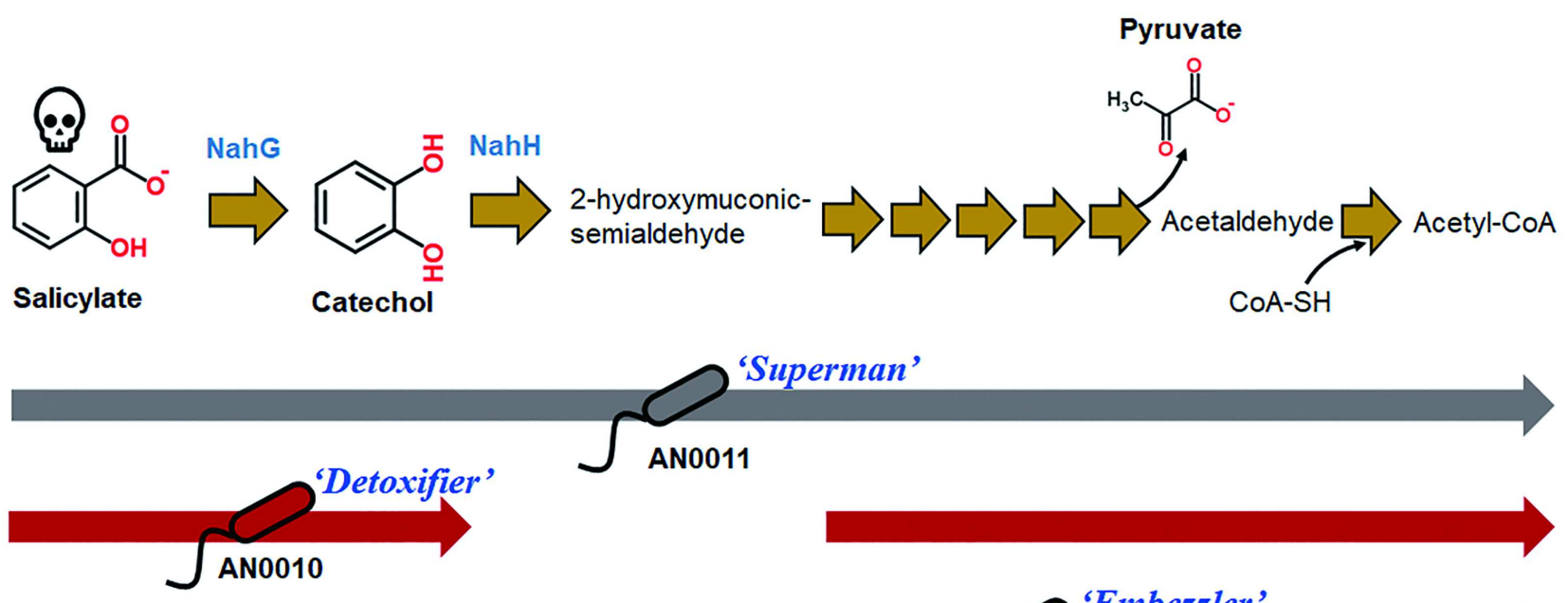

\section{'Superman'}

AN0011

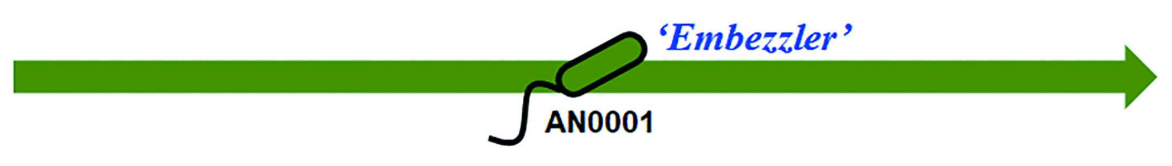

B
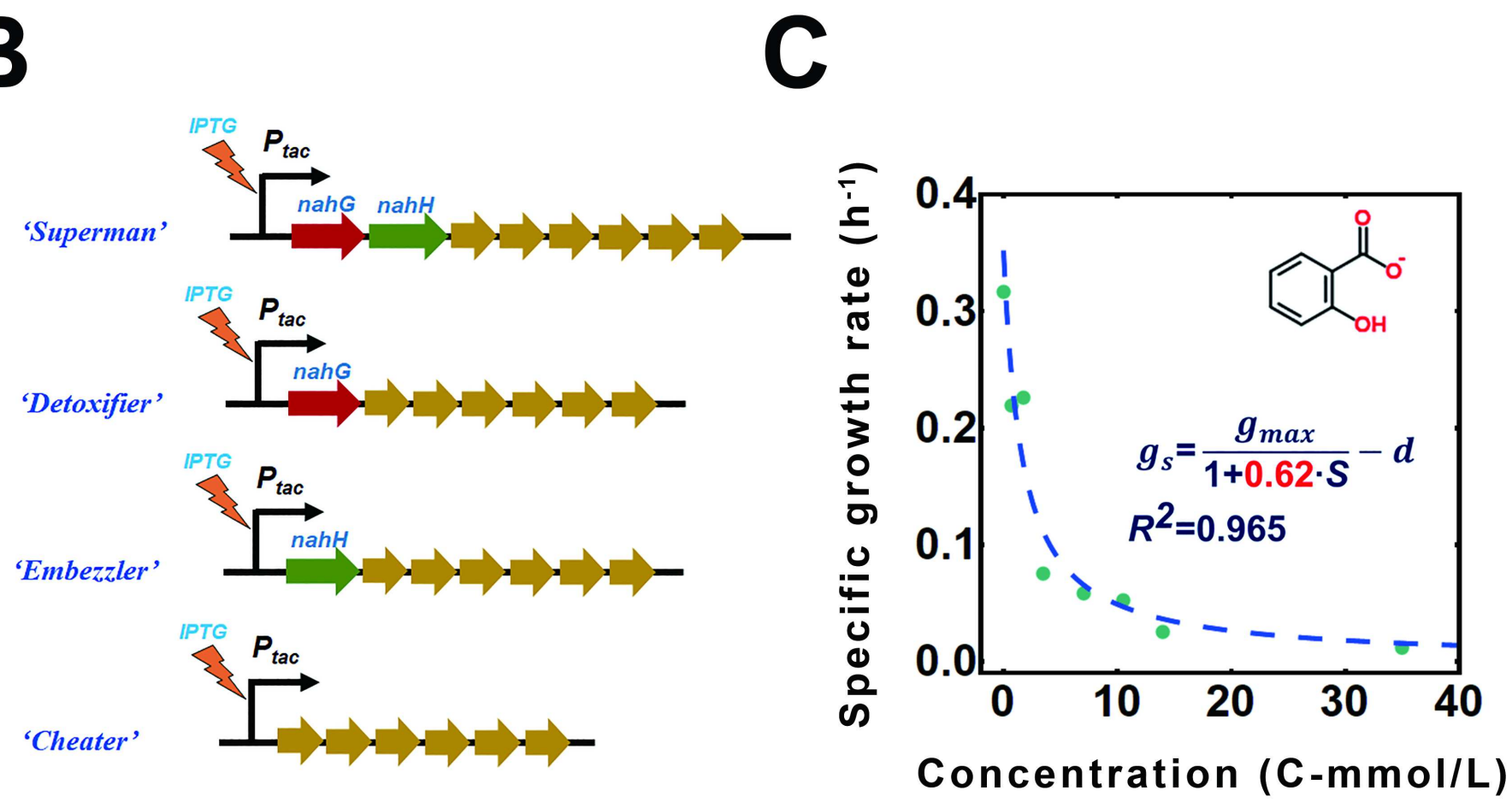

Figure 3

Design of the SMC-mdol. (A) Pathway of salicylate degradation in 'Superman' strain P. stutzeri AN0011, as well as partial pathways carried out by Detoxifier strain AN0010 and Embezzler strain AN0001. Skull marks that salicylate is toxic. (B) Diagram of operons responsible for the salicylate degradation, located in the chromosomes of AN0011, AN0010, AN0001 as well as a homologous mutant AN0000. (C) 
Determination of the toxic strength of the substrate salicylate. We measured the growth rate of AN0000 using pyruvate, one of the end products, as the carbon source, and supplying different concentration of salicylate. The growth rates (g_s) were fitted with the corresponding salicylate concentration (S), using a formula involving maximum growth rate (g_max) and one-dimensional death rate (d), showing a hyperbolic toxic effect (See Methods section for detailed description).

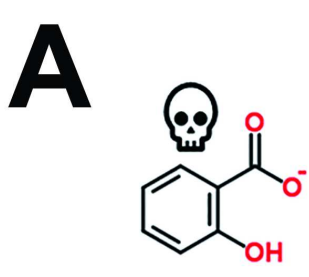

Salicylate
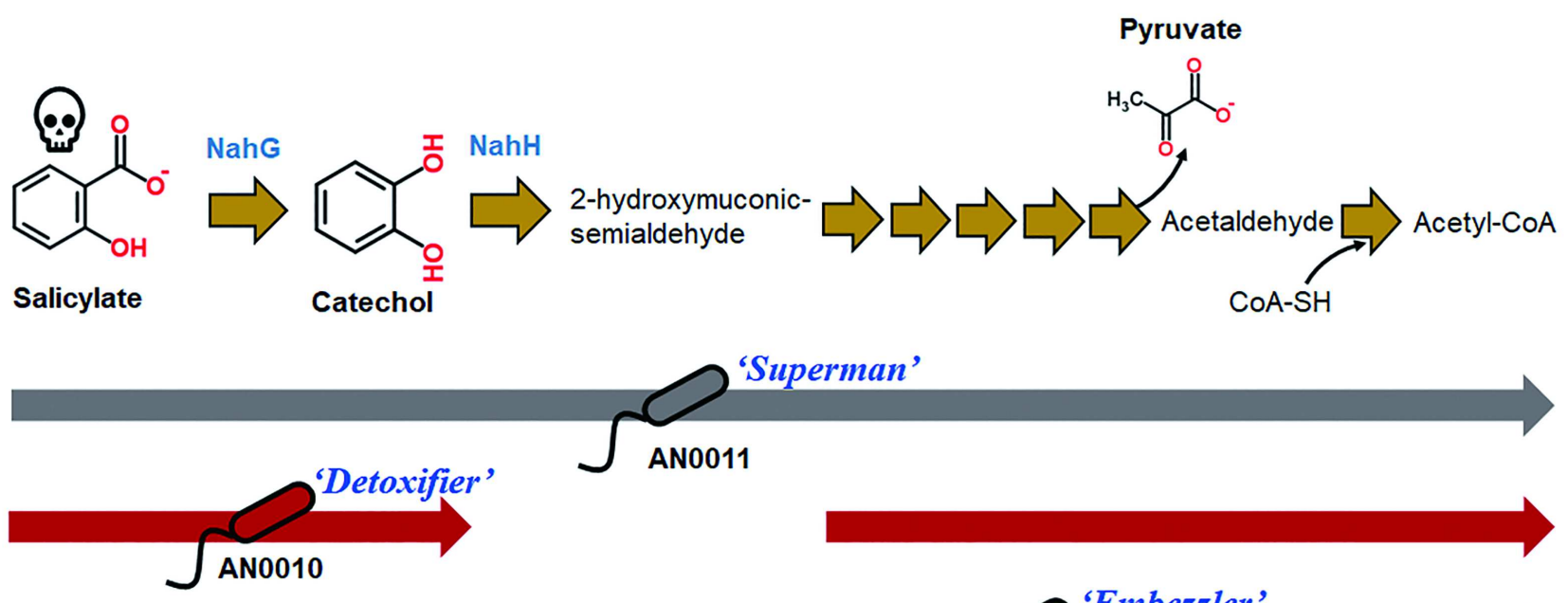

\section{'Superman'}

AN0011

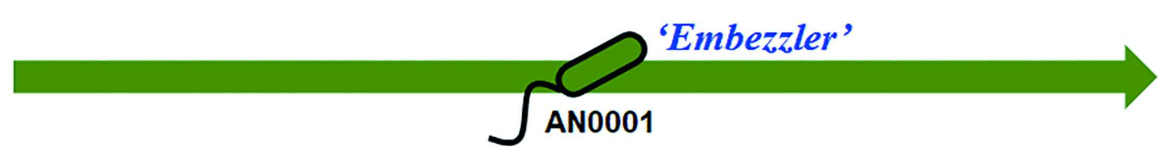

B
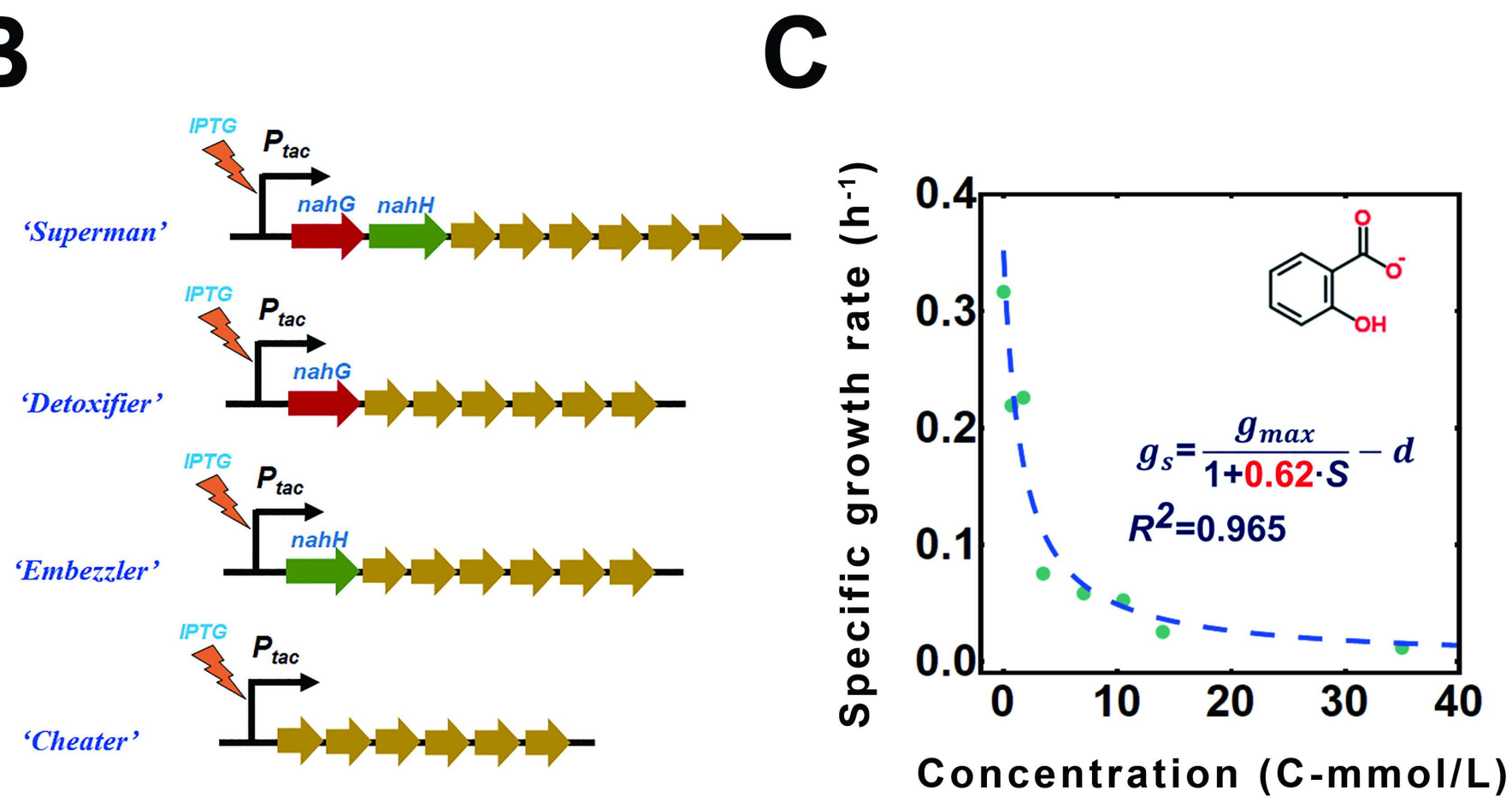

Figure 3

Design of the SMC-mdol. (A) Pathway of salicylate degradation in 'Superman' strain P. stutzeri AN0011, as well as partial pathways carried out by Detoxifier strain AN0010 and Embezzler strain AN0001. Skull marks that salicylate is toxic. (B) Diagram of operons responsible for the salicylate degradation, located in the chromosomes of AN0011, AN0010, AN0001 as well as a homologous mutant AN0000. (C) 
Determination of the toxic strength of the substrate salicylate. We measured the growth rate of AN0000 using pyruvate, one of the end products, as the carbon source, and supplying different concentration of salicylate. The growth rates (g_s) were fitted with the corresponding salicylate concentration (S), using a formula involving maximum growth rate (g_max) and one-dimensional death rate (d), showing a hyperbolic toxic effect (See Methods section for detailed description).

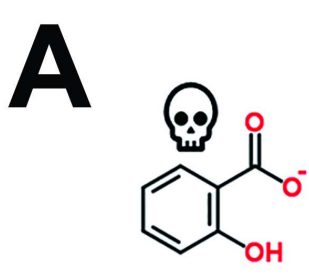

Salicylate
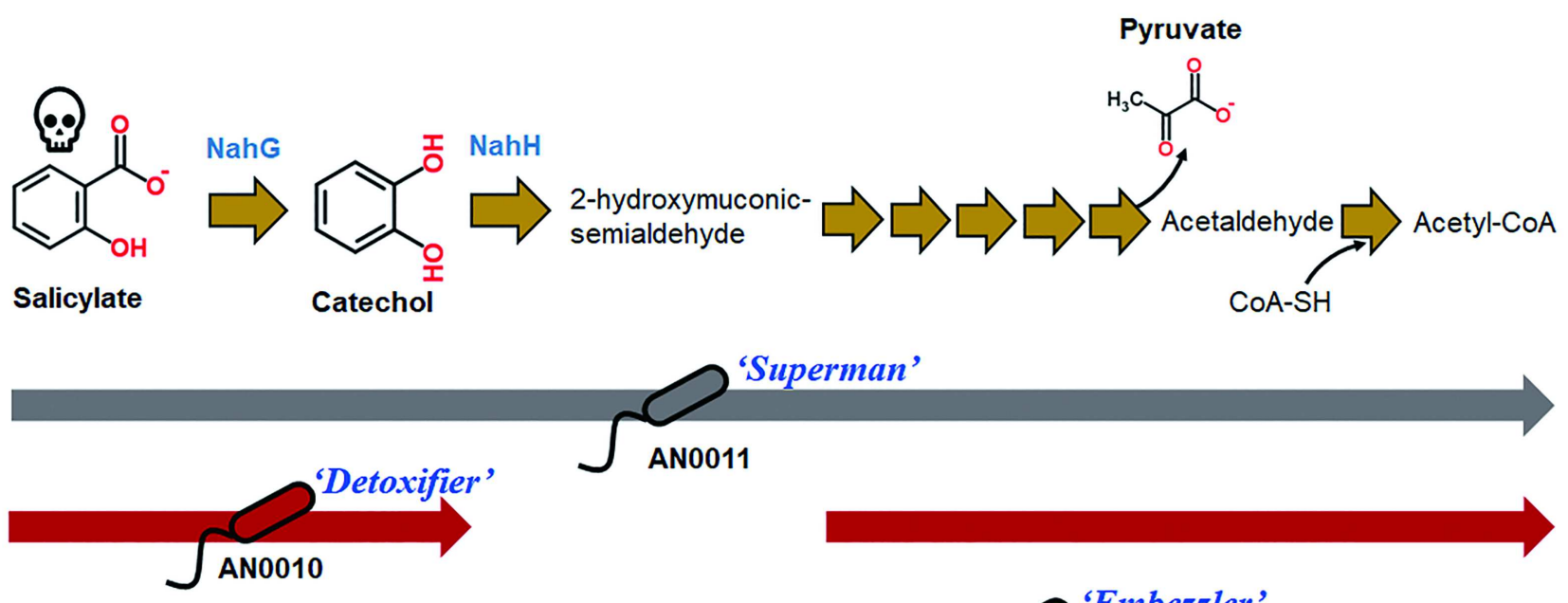

\section{'Superman'}

AN0011

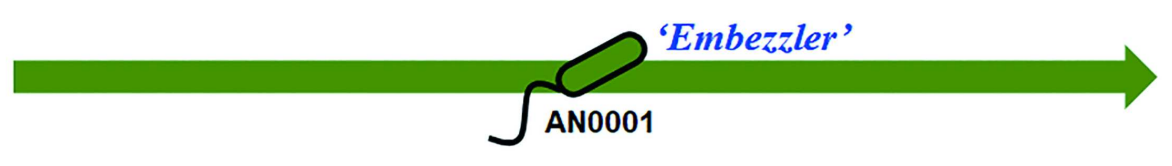

B
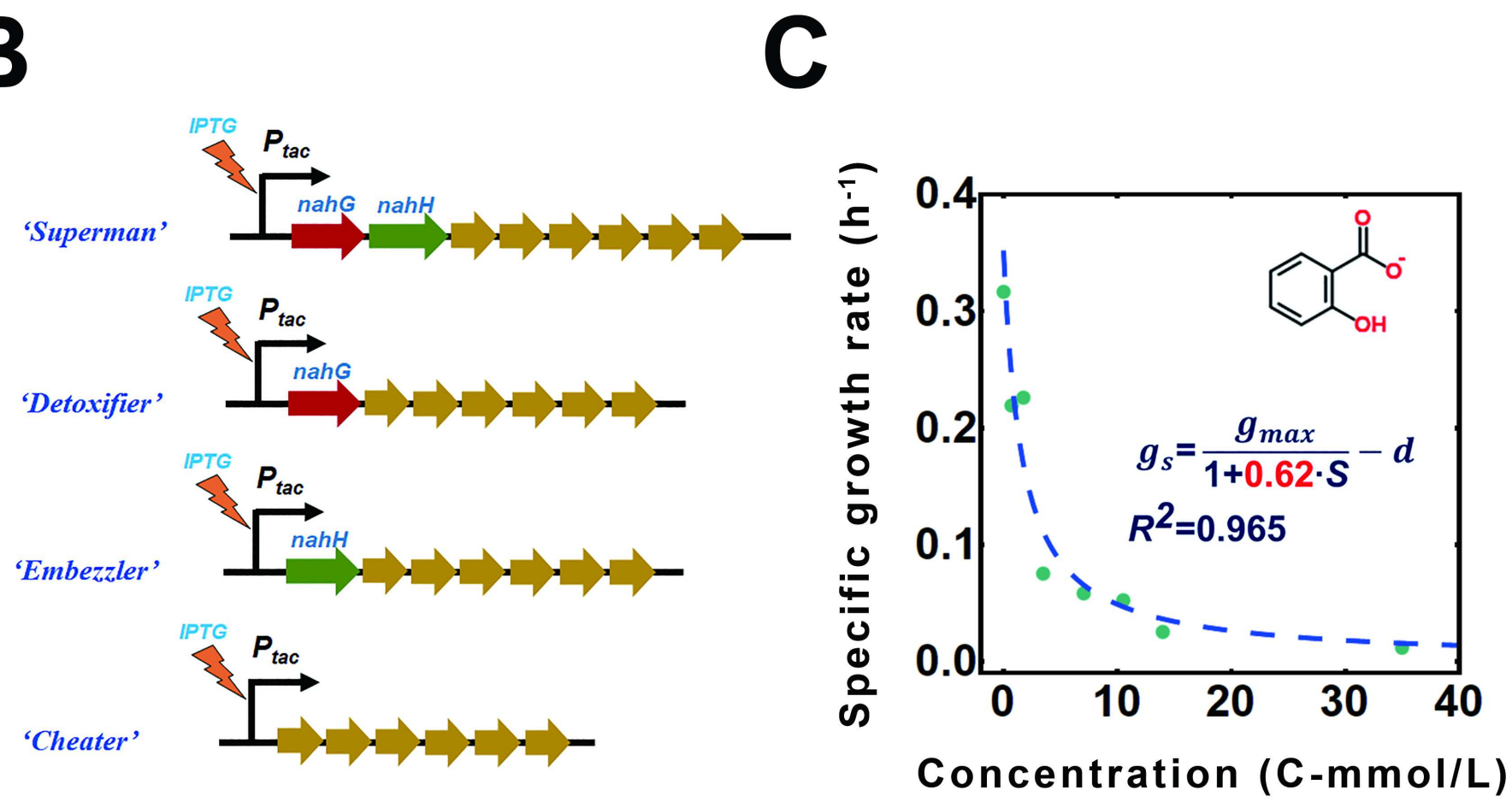

Figure 3

Design of the SMC-mdol. (A) Pathway of salicylate degradation in 'Superman' strain P. stutzeri AN0011, as well as partial pathways carried out by Detoxifier strain AN0010 and Embezzler strain AN0001. Skull marks that salicylate is toxic. (B) Diagram of operons responsible for the salicylate degradation, located in the chromosomes of AN0011, AN0010, AN0001 as well as a homologous mutant AN0000. (C) 
Determination of the toxic strength of the substrate salicylate. We measured the growth rate of AN0000 using pyruvate, one of the end products, as the carbon source, and supplying different concentration of salicylate. The growth rates (g_s) were fitted with the corresponding salicylate concentration (S), using a formula involving maximum growth rate (g_max) and one-dimensional death rate (d), showing a hyperbolic toxic effect (See Methods section for detailed description).

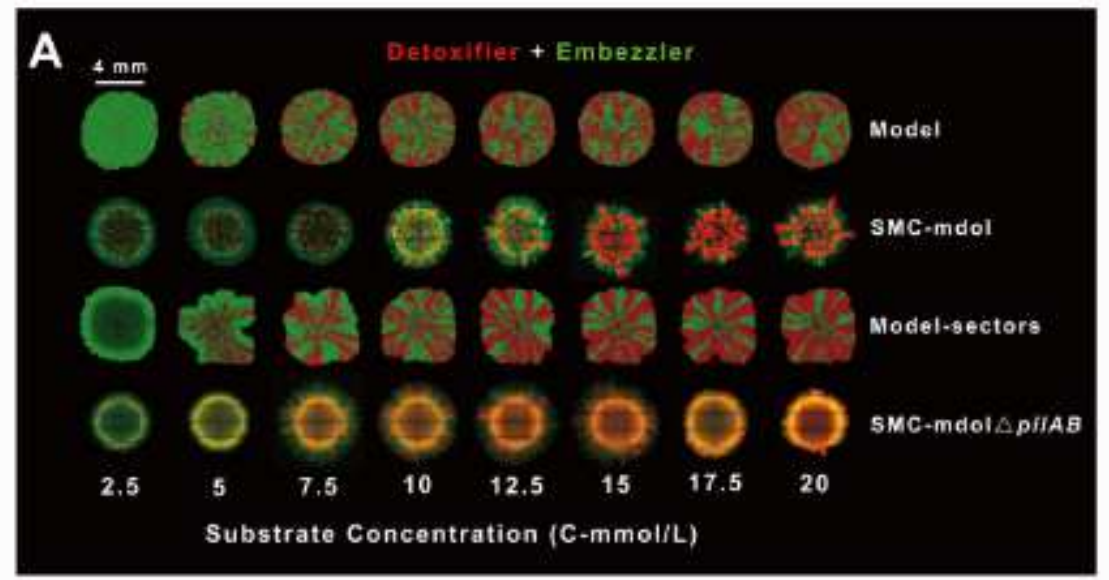

B
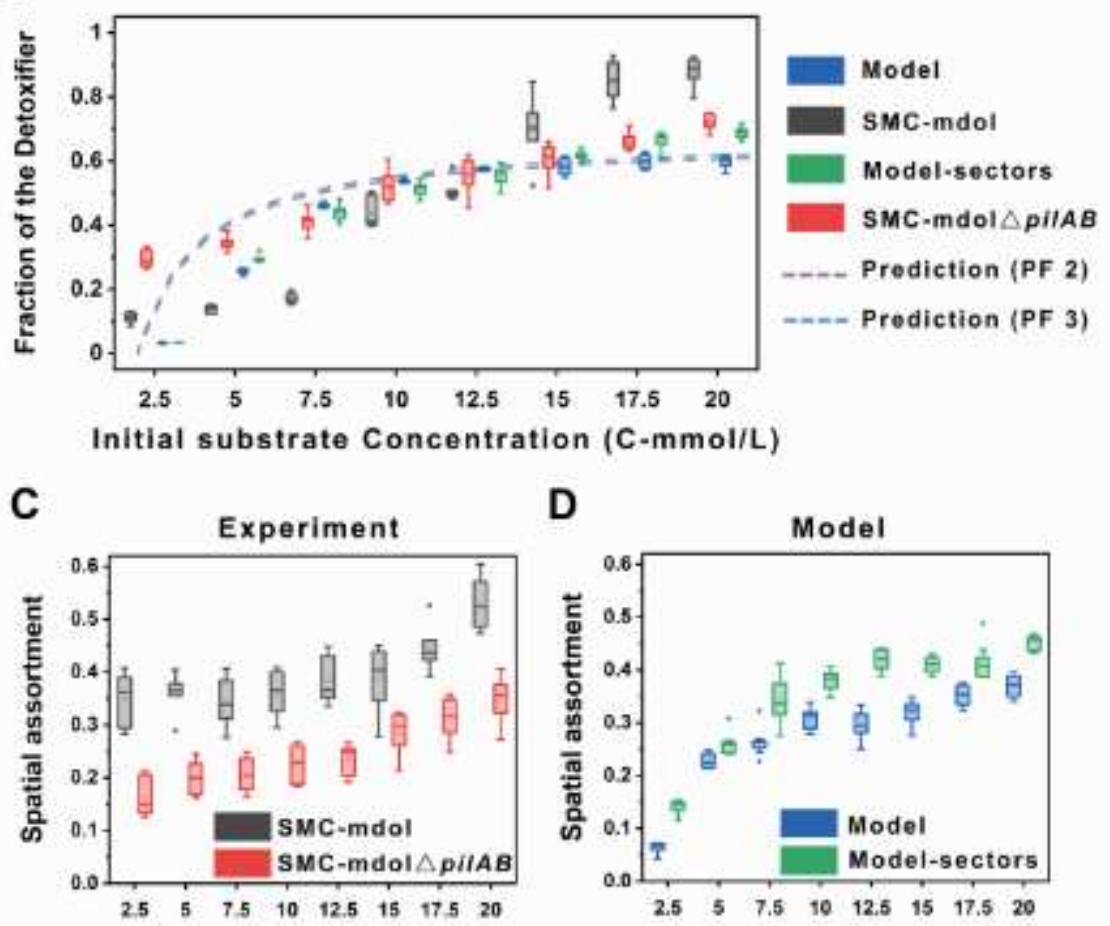

Initial substrate Concentration (C-mmol/L) Initial substrate Concentration (C-mmol/h)

\section{Figure 4}

Substrate concentration and toxicity govern the assembly and spatial organization of the SMC-mdol and SMC-mdol $\triangle$ pilAB. (A) Representative colony patterns from the pattern formation assays of SMC-mdol and SMC-mdol囚pilAB, as well as the individual-based simulations with the modified model according to experimental parameters (denoted as 'Model') and the model with additional limited resource, $\mathrm{L}$ (denoted as 'Model-sectors'; see Supplementary information S1 for detail about the modifications of these models), across eight different initial substrate concentrations. (B) Analysis of community composition of the experimental formed colonies, as well as the model predicted colonies across eight kinds of initial 
substrate concentrations. The purple dashed line indicates the relative fraction of 'Detoxifier' calculated from the PF2, while the blue dashed line indicates the relative fraction of 'Detoxifier' calculated from the PF3. We use adjusted R2 to quantify the prediction power, where PF2 shows a power of 0.51 for the assembly of SMC-mdol and 0.76 for that of SMC-mdol $\triangle$ pilAB, while PF3 shows a power of 0.52 for the assembly of SMC-mdol and 0.78 for that of SMC-mdol『pilAB. (C-D) Analysis of spatial assortment experimental patterns (C) and the model predicted patterns (D) across eight different initial substrate concentrations. Note the absolute values of spatial assortment from the experiments and simulations are incomparable due to the difference between their scales (means experimental patterns containing more cells) and calculation methods (See Methods and Supplementary Information S1 sections for detail), but the trends of variation across the substrate concentrations are the same. For experiments, six replicates were performed for each condition, while for simulations, eight replicates were performed.

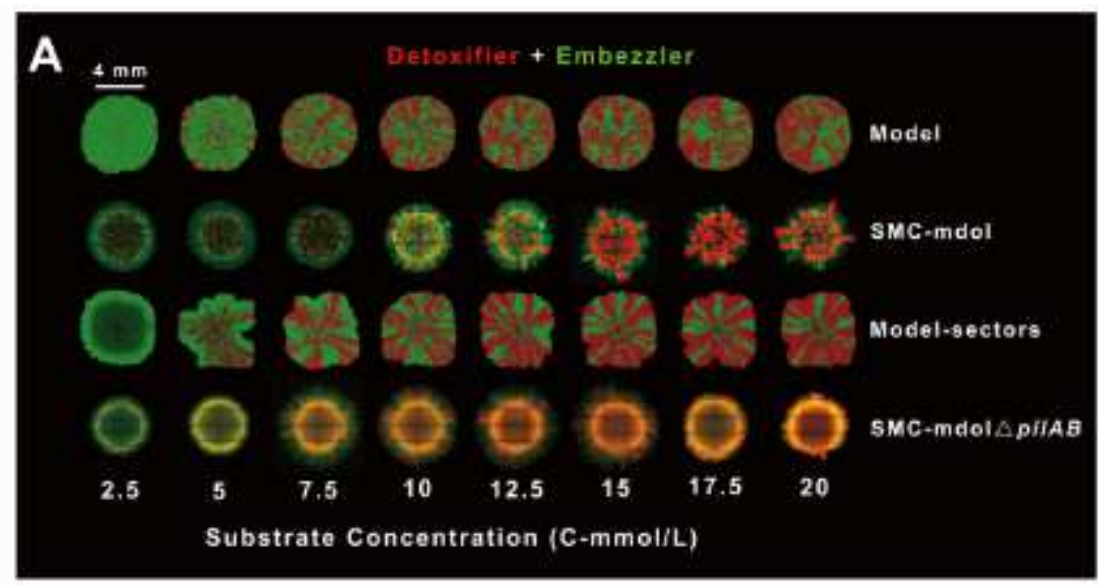

B
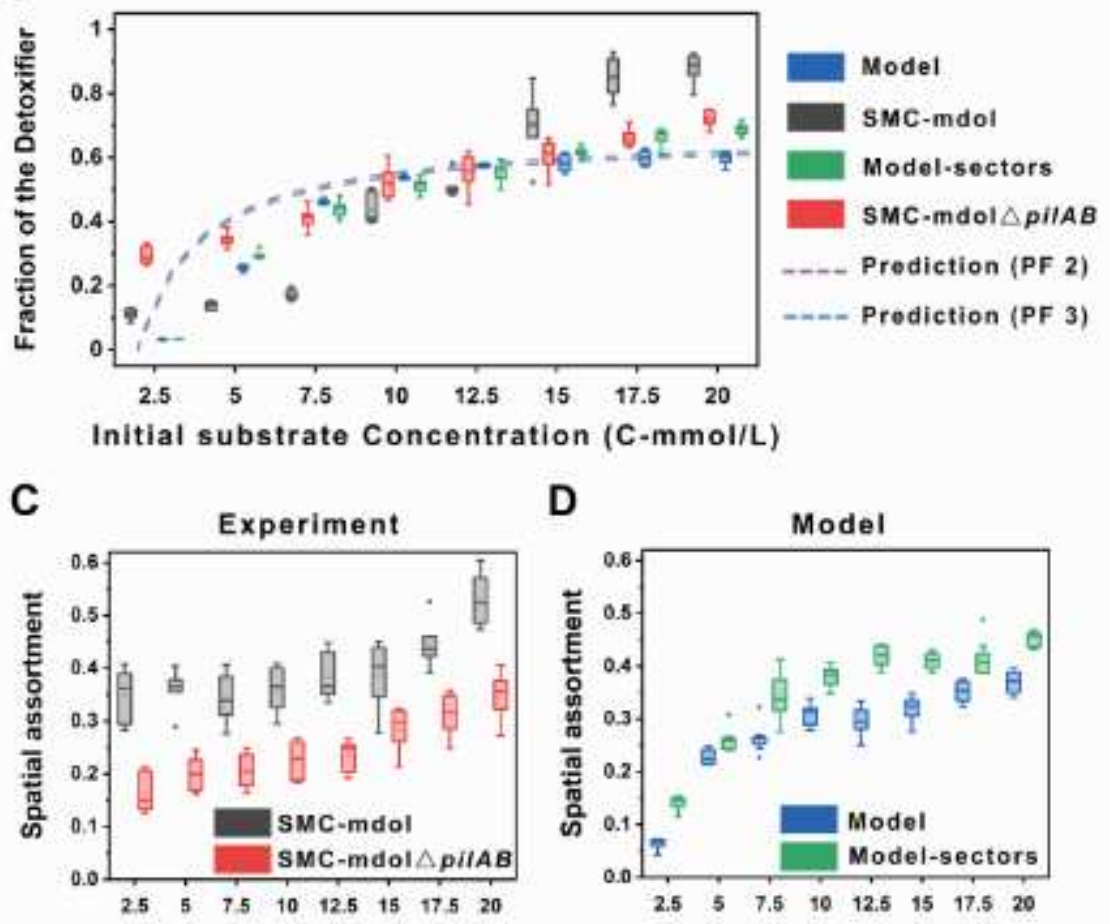

Initial substrate Concentration (C-mmol/L) Initial substrate Concentration (C-mmolnL)

\section{Figure 4}


Substrate concentration and toxicity govern the assembly and spatial organization of the SMC-mdol and SMC-mdol $\triangle$ pilAB. (A) Representative colony patterns from the pattern formation assays of SMC-mdol and SMC-mdol囚pilAB, as well as the individual-based simulations with the modified model according to experimental parameters (denoted as 'Model') and the model with additional limited resource, $L$ (denoted as 'Model-sectors'; see Supplementary information S1 for detail about the modifications of these models), across eight different initial substrate concentrations. (B) Analysis of community composition of the experimental formed colonies, as well as the model predicted colonies across eight kinds of initial substrate concentrations. The purple dashed line indicates the relative fraction of 'Detoxifier' calculated from the PF2, while the blue dashed line indicates the relative fraction of 'Detoxifier' calculated from the PF3. We use adjusted R2 to quantify the prediction power, where PF2 shows a power of 0.51 for the assembly of SMC-mdol and 0.76 for that of SMC-mdol $\triangle$ pilAB, while PF3 shows a power of 0.52 for the assembly of SMC-mdol and 0.78 for that of SMC-mdol『pilAB. (C-D) Analysis of spatial assortment experimental patterns (C) and the model predicted patterns (D) across eight different initial substrate concentrations. Note the absolute values of spatial assortment from the experiments and simulations are incomparable due to the difference between their scales (means experimental patterns containing more cells) and calculation methods (See Methods and Supplementary Information S1 sections for detail), but the trends of variation across the substrate concentrations are the same. For experiments, six replicates were performed for each condition, while for simulations, eight replicates were performed. 


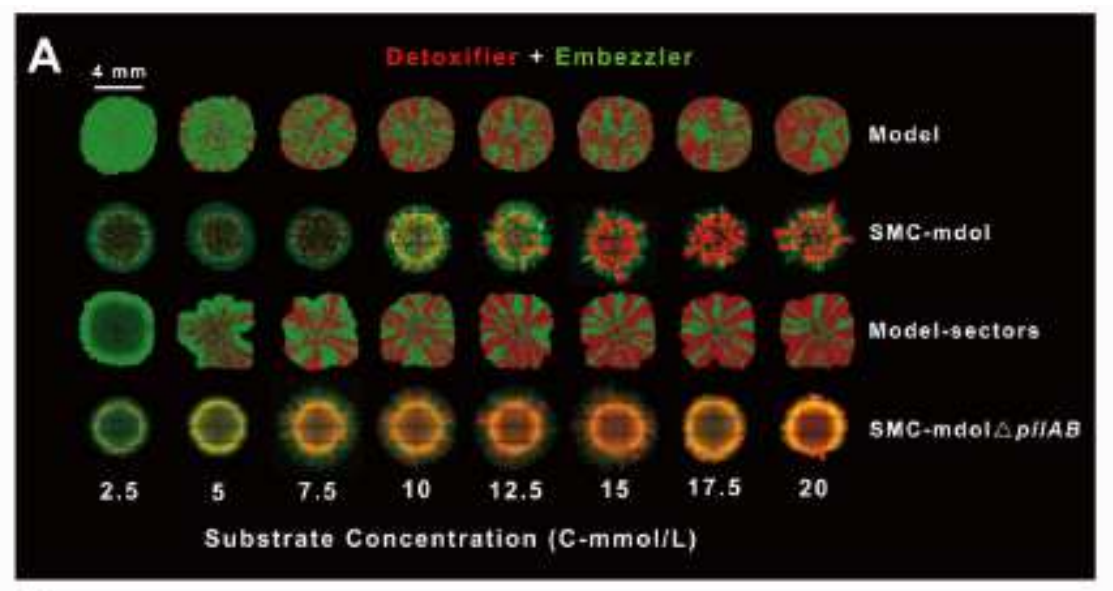

B

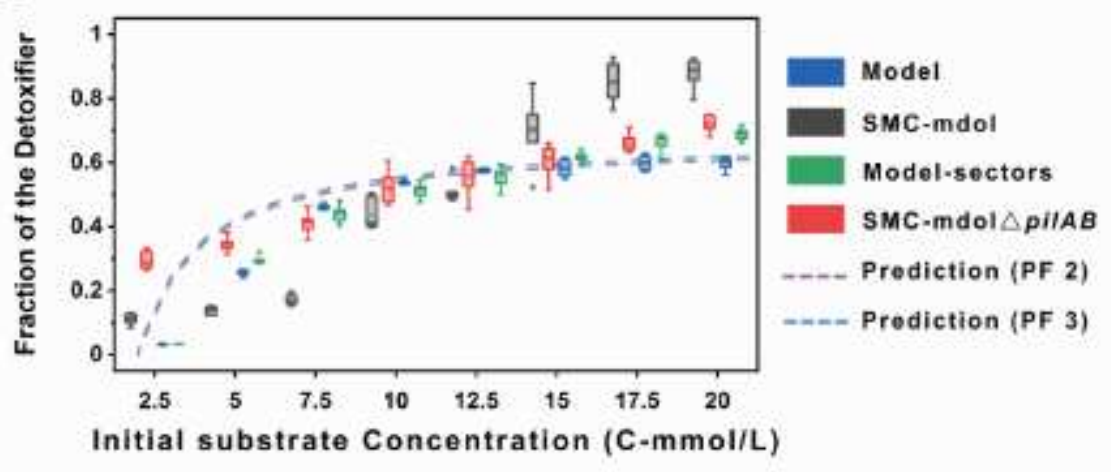

C

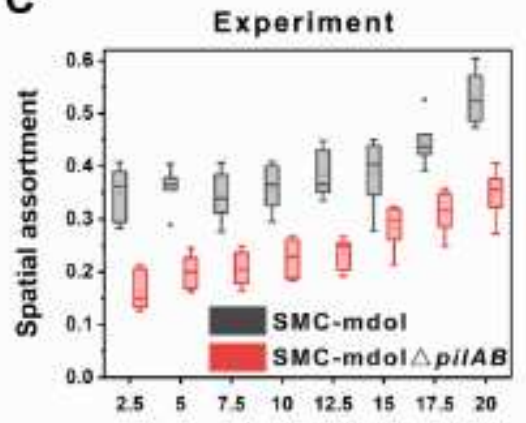

D Model

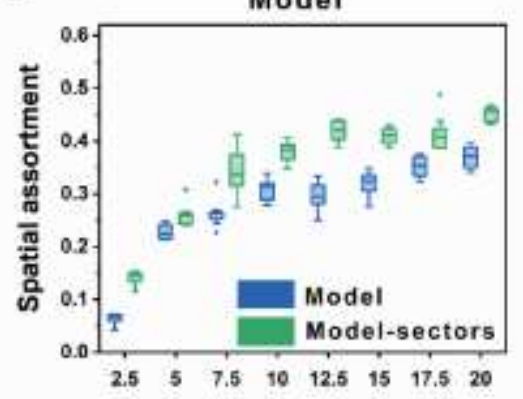

Initial substrate Concentration (C-mmol/L) Initial substrate Concentration (C-mmoln $\mathrm{L}$ )

\section{Figure 4}

Substrate concentration and toxicity govern the assembly and spatial organization of the SMC-mdol and SMC-mdol $\triangle$ pilAB. (A) Representative colony patterns from the pattern formation assays of SMC-mdol and SMC-mdol $\triangle$ pilAB, as well as the individual-based simulations with the modified model according to experimental parameters (denoted as 'Model') and the model with additional limited resource, $L$ (denoted as 'Model-sectors'; see Supplementary information S1 for detail about the modifications of these models), across eight different initial substrate concentrations. (B) Analysis of community composition of the experimental formed colonies, as well as the model predicted colonies across eight kinds of initial substrate concentrations. The purple dashed line indicates the relative fraction of 'Detoxifier' calculated from the PF2, while the blue dashed line indicates the relative fraction of 'Detoxifier' calculated from the PF3. We use adjusted R2 to quantify the prediction power, where PF2 shows a power of 0.51 for the assembly of SMC-mdol and 0.76 for that of SMC-mdol $\triangle$ pilAB, while PF3 shows a power of 0.52 for the assembly of SMC-mdol and 0.78 for that of SMC-mdol『pilAB. (C-D) Analysis of spatial assortment 
experimental patterns (C) and the model predicted patterns (D) across eight different initial substrate concentrations. Note the absolute values of spatial assortment from the experiments and simulations are incomparable due to the difference between their scales (means experimental patterns containing more cells) and calculation methods (See Methods and Supplementary Information S1 sections for detail), but the trends of variation across the substrate concentrations are the same. For experiments, six replicates were performed for each condition, while for simulations, eight replicates were performed.

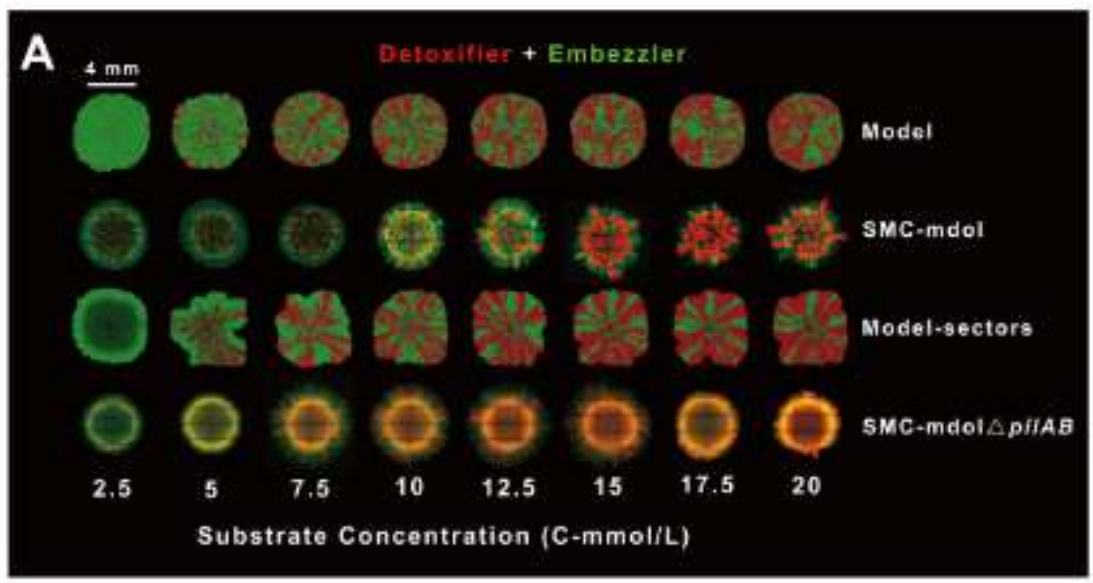

B

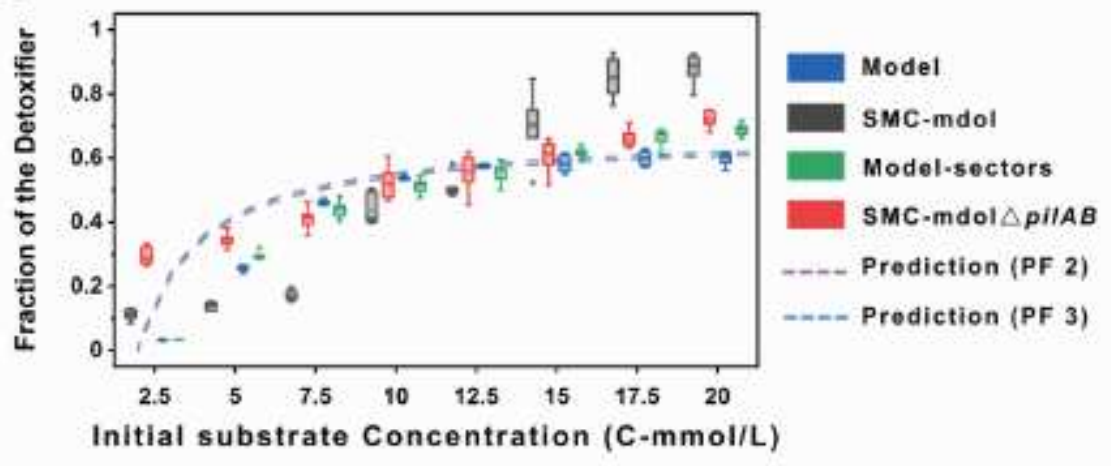

C

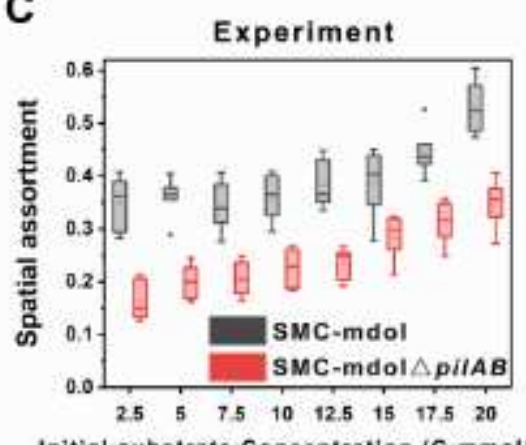

Dodel

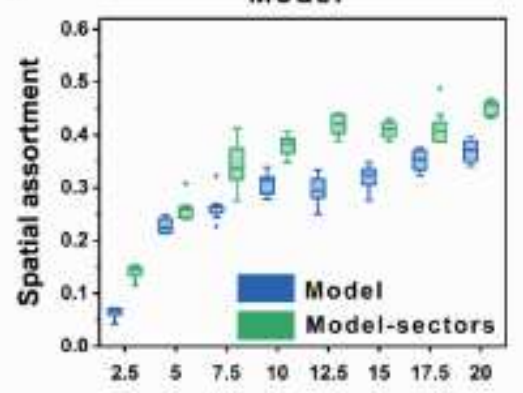

Initial substrate Concentration (C-mmol/L) Initial substrate Concentration (C-mmoln )

\section{Figure 4}

Substrate concentration and toxicity govern the assembly and spatial organization of the SMC-mdol and SMC-mdol $\triangle$ pilAB. (A) Representative colony patterns from the pattern formation assays of SMC-mdol and SMC-mdol『pilAB, as well as the individual-based simulations with the modified model according to experimental parameters (denoted as 'Model') and the model with additional limited resource, $\mathrm{L}$ (denoted as 'Model-sectors'; see Supplementary information S1 for detail about the modifications of these models), across eight different initial substrate concentrations. (B) Analysis of community composition 
of the experimental formed colonies, as well as the model predicted colonies across eight kinds of initial substrate concentrations. The purple dashed line indicates the relative fraction of 'Detoxifier' calculated from the PF2, while the blue dashed line indicates the relative fraction of 'Detoxifier' calculated from the PF3. We use adjusted R2 to quantify the prediction power, where PF2 shows a power of 0.51 for the assembly of SMC-mdol and 0.76 for that of SMC-mdol $\triangle$ pilAB, while PF3 shows a power of 0.52 for the assembly of SMC-mdol and 0.78 for that of SMC-mdol囚pilAB. (C-D) Analysis of spatial assortment experimental patterns (C) and the model predicted patterns (D) across eight different initial substrate concentrations. Note the absolute values of spatial assortment from the experiments and simulations are incomparable due to the difference between their scales (means experimental patterns containing more cells) and calculation methods (See Methods and Supplementary Information S1 sections for detail), but the trends of variation across the substrate concentrations are the same. For experiments, six replicates were performed for each condition, while for simulations, eight replicates were performed.

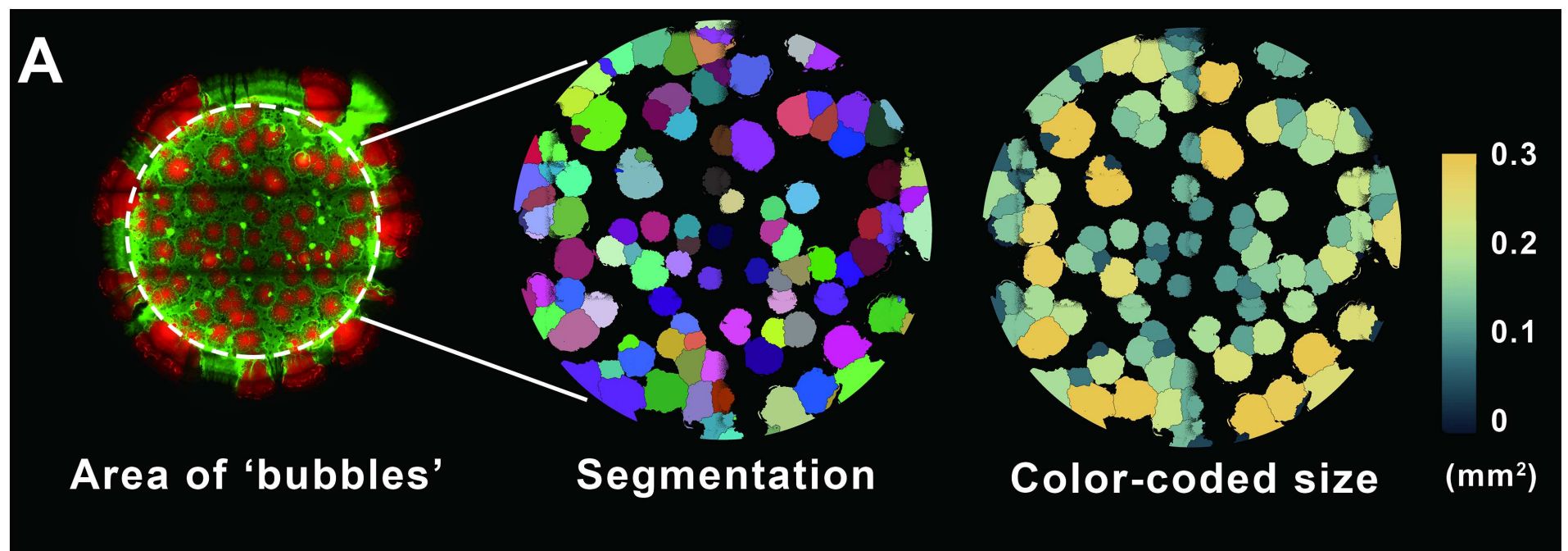

B

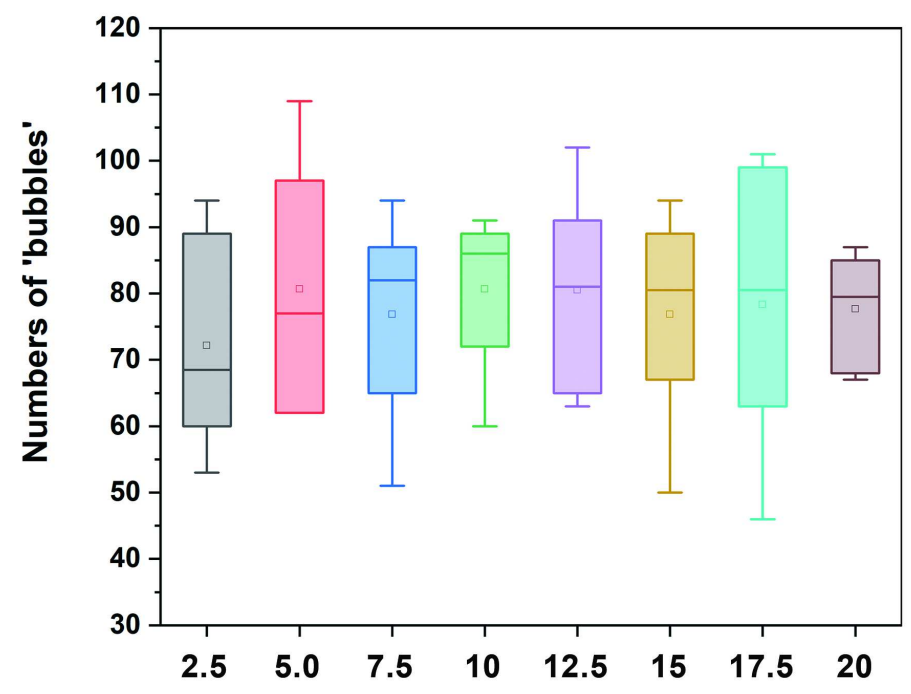

Substrate concentration (C-mmol/L)

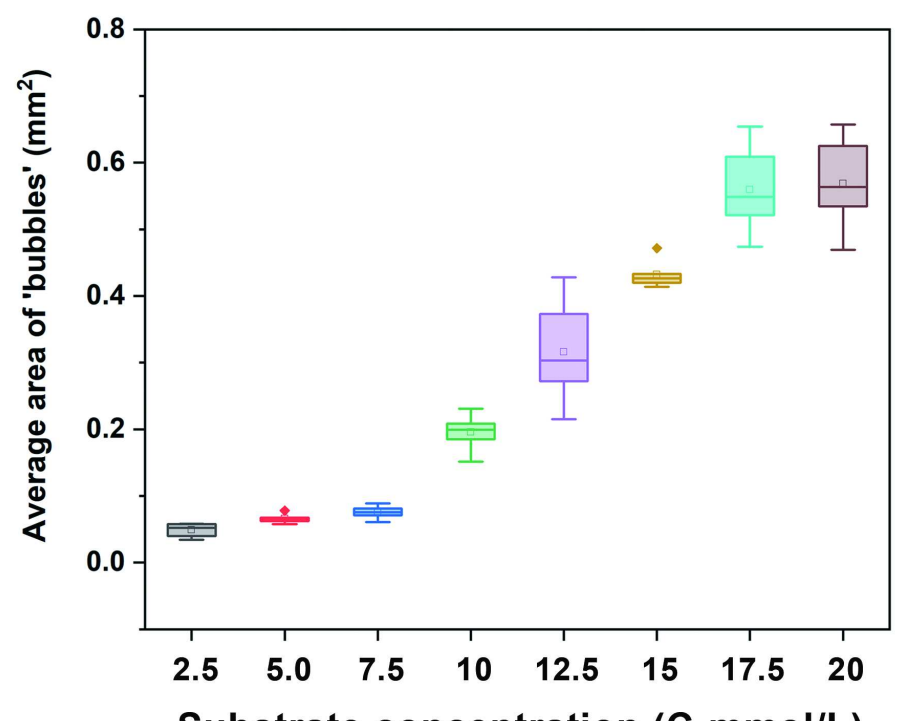

Substrate concentration (C-mmol/L) 
Substrate concentration governs the geometry of the 'bubble' structures inside the spatial patterns developed by SMC-mdol. (A) Workflow of the image analysis of the 'bubble' area. 'bubbles' formed by Detoxifier cells were segmented and analyzed to get its area size $(\mathrm{mm} 2)$. In the right graph, bubbles are color-coded based on their individual area size, with brighter colors indicating larger sizes. (B) Average number of the bubbles in the colony formed by SMC-mdol in different initial substrate concentration. (C) Average area size of the bubbles inside the colony formed by SMC-mdol in different initial substrate concentration. Images used here is same as Figure 4.

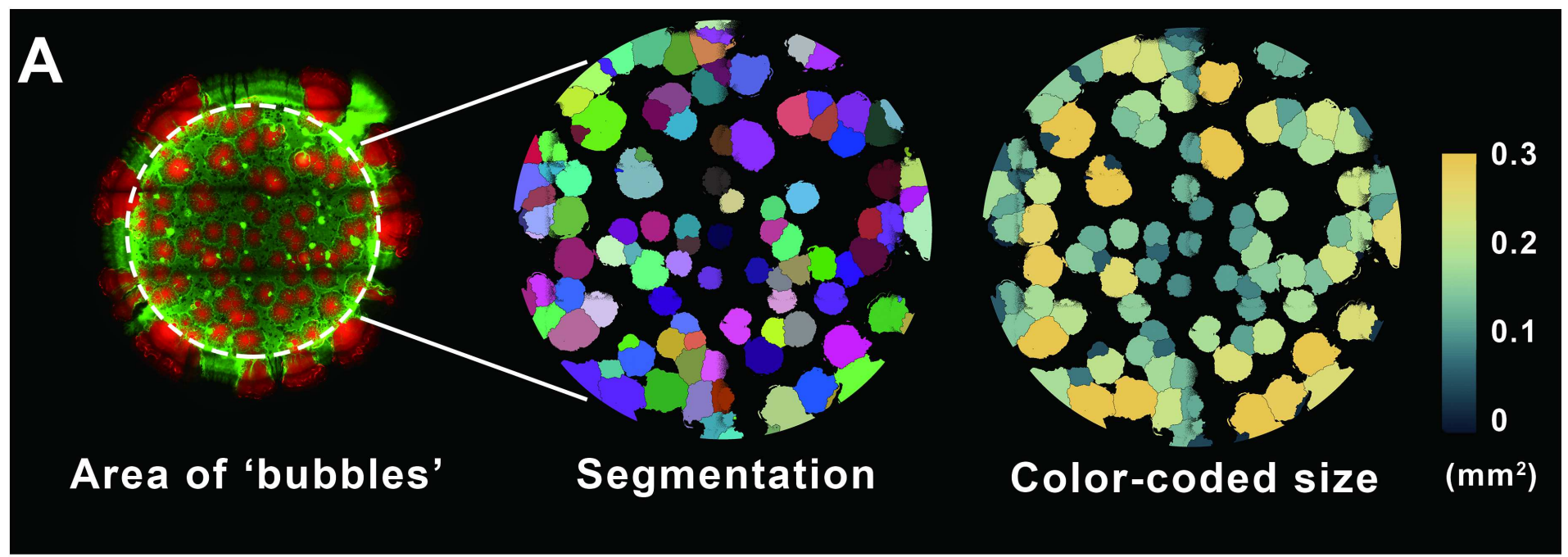

B
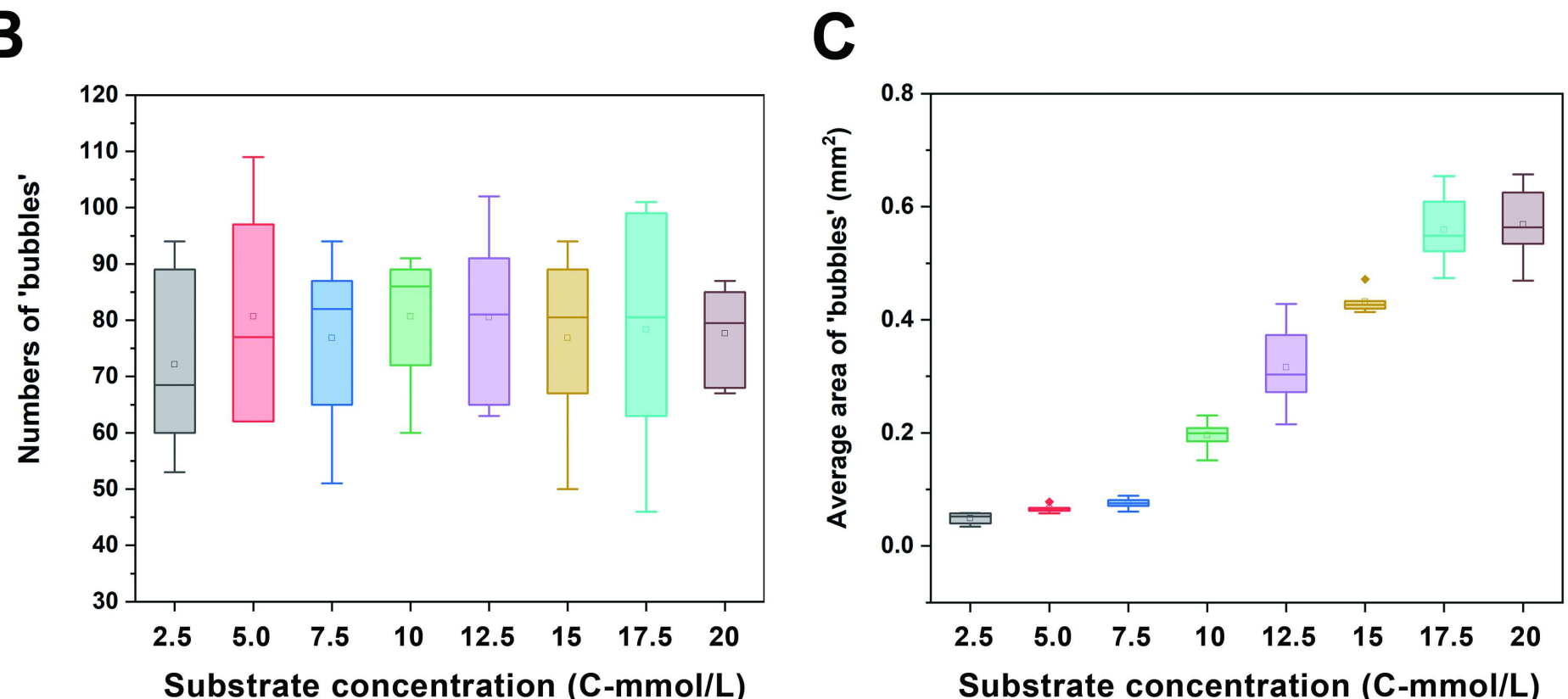

Substrate concentration (C-mmol/L)

Figure 5

Substrate concentration governs the geometry of the 'bubble' structures inside the spatial patterns developed by SMC-mdol. (A) Workflow of the image analysis of the 'bubble' area. 'bubbles' formed by Detoxifier cells were segmented and analyzed to get its area size $(\mathrm{mm} 2)$. In the right graph, bubbles are color-coded based on their individual area size, with brighter colors indicating larger sizes. (B) Average 
number of the bubbles in the colony formed by SMC-mdol in different initial substrate concentration. (C) Average area size of the bubbles inside the colony formed by SMC-mdol in different initial substrate concentration. Images used here is same as Figure 4.

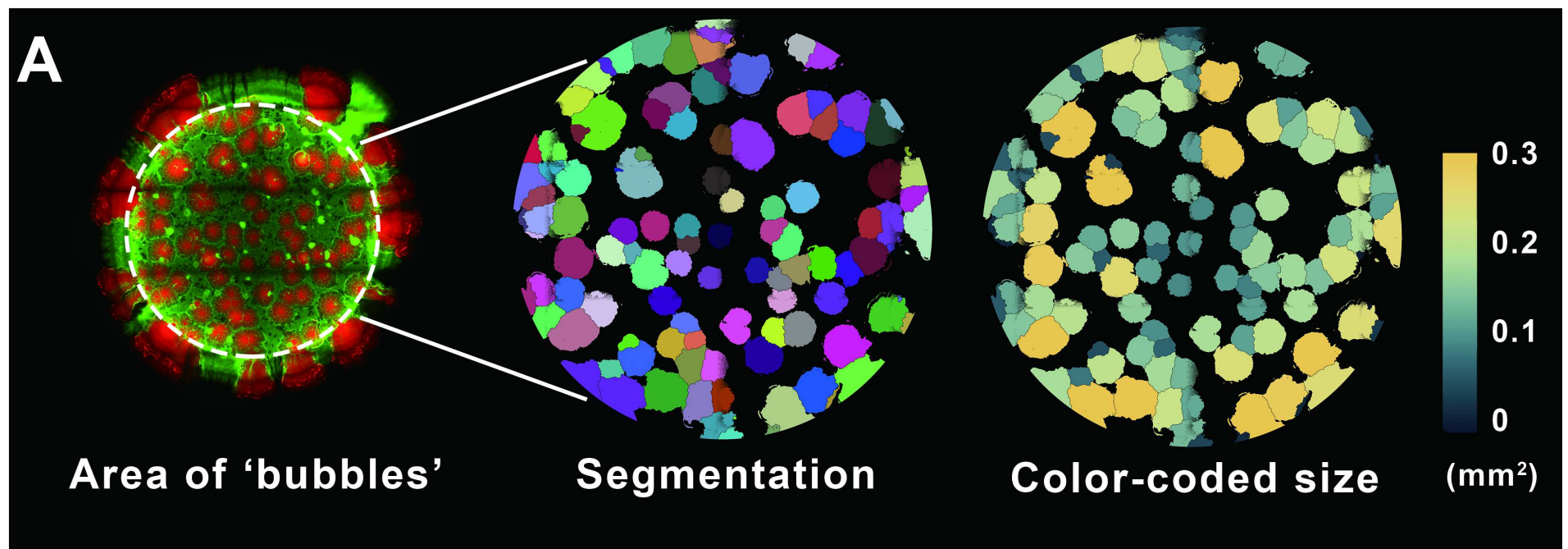

B
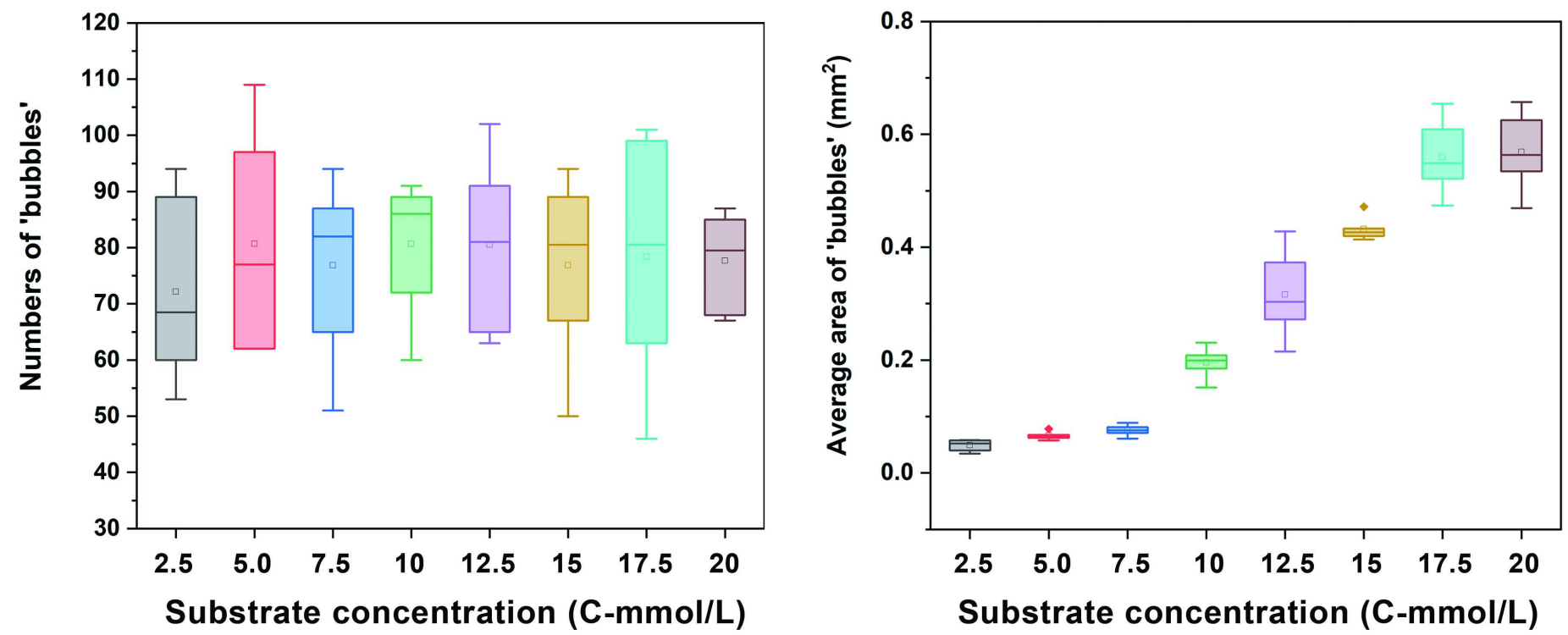

Figure 5

Substrate concentration governs the geometry of the 'bubble' structures inside the spatial patterns developed by SMC-mdol. (A) Workflow of the image analysis of the 'bubble' area. 'bubbles' formed by Detoxifier cells were segmented and analyzed to get its area size $(\mathrm{mm} 2)$. In the right graph, bubbles are color-coded based on their individual area size, with brighter colors indicating larger sizes. (B) Average number of the bubbles in the colony formed by SMC-mdol in different initial substrate concentration. (C) Average area size of the bubbles inside the colony formed by SMC-mdol in different initial substrate concentration. Images used here is same as Figure 4. 


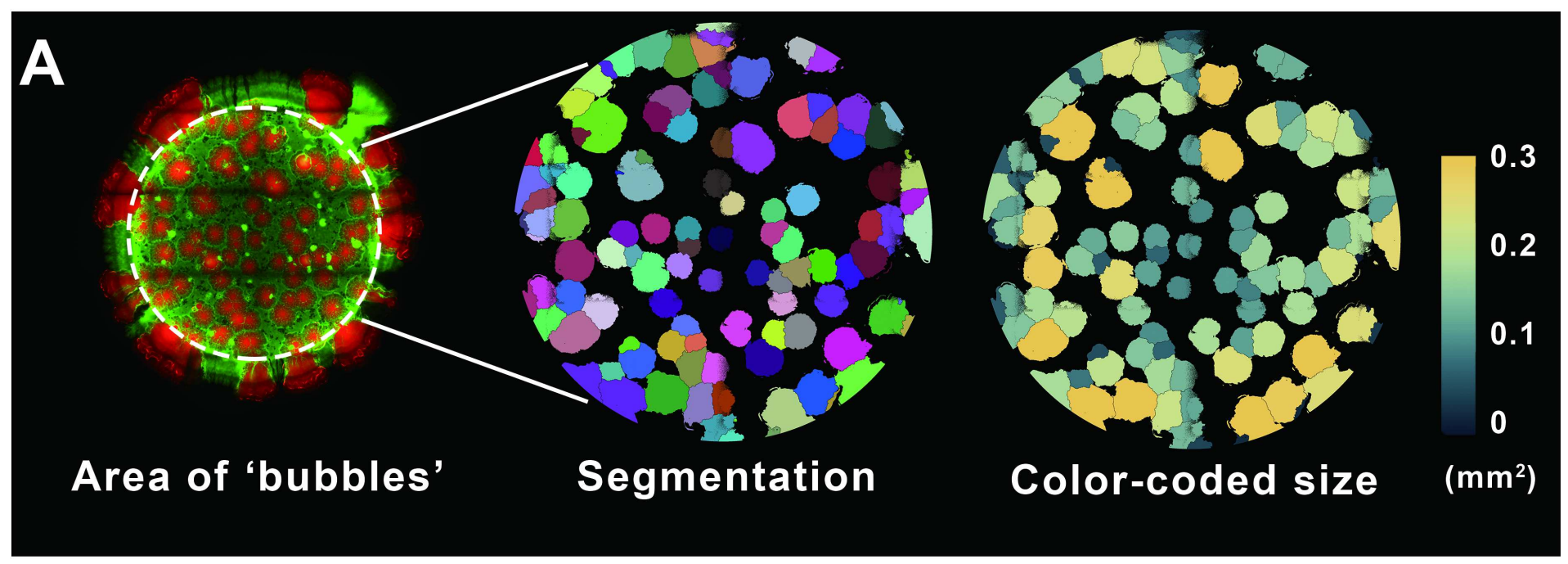

B
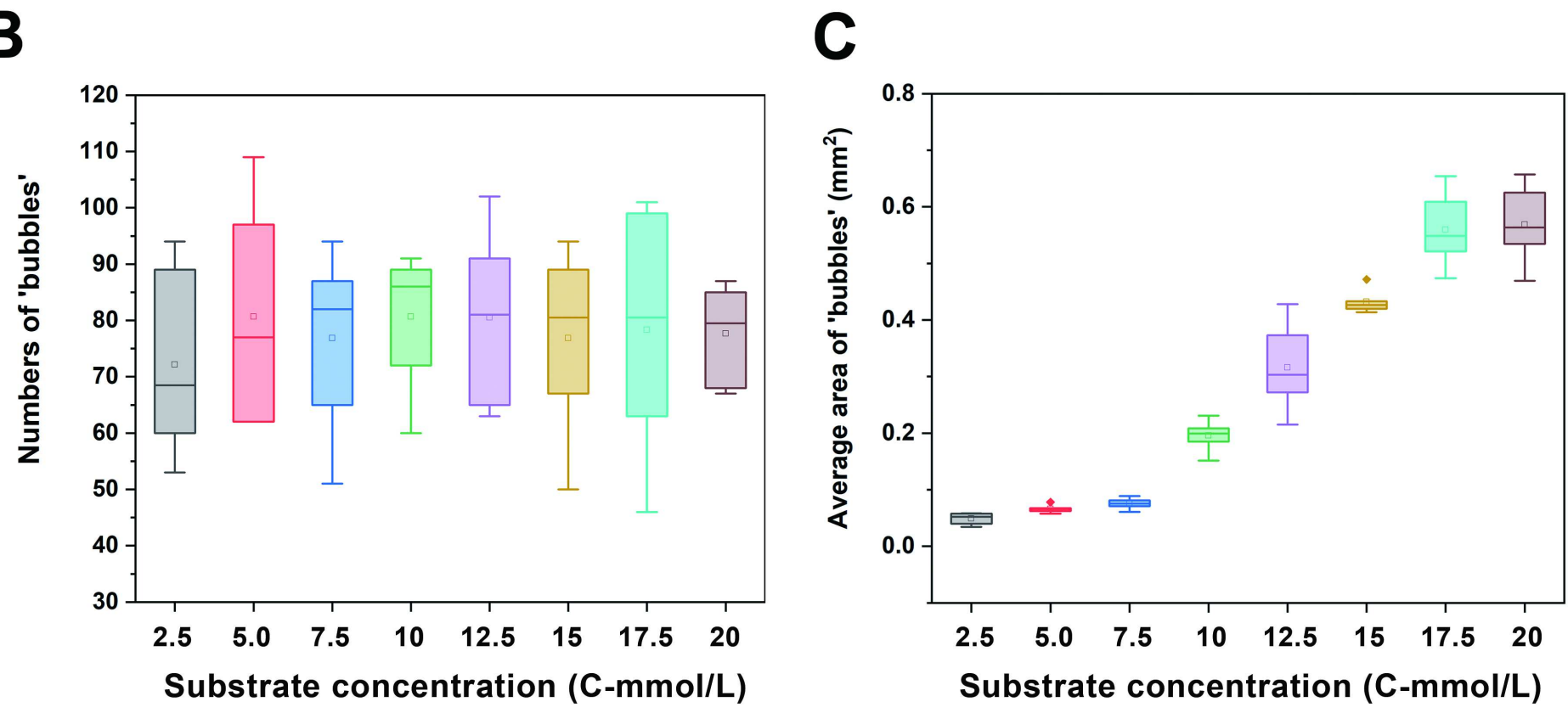

Figure 5

Substrate concentration governs the geometry of the 'bubble' structures inside the spatial patterns developed by SMC-mdol. (A) Workflow of the image analysis of the 'bubble' area. 'bubbles' formed by Detoxifier cells were segmented and analyzed to get its area size $(\mathrm{mm} 2)$. In the right graph, bubbles are color-coded based on their individual area size, with brighter colors indicating larger sizes. (B) Average number of the bubbles in the colony formed by SMC-mdol in different initial substrate concentration. (C) Average area size of the bubbles inside the colony formed by SMC-mdol in different initial substrate concentration. Images used here is same as Figure 4. 


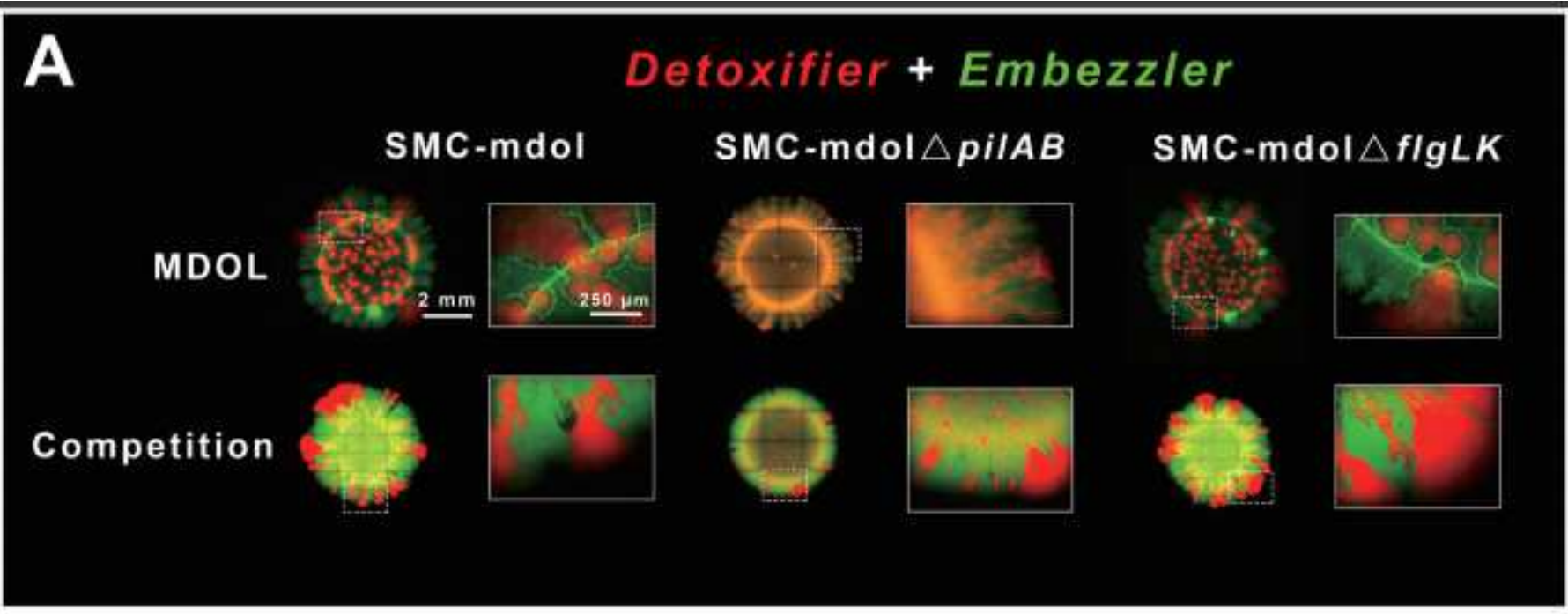

B
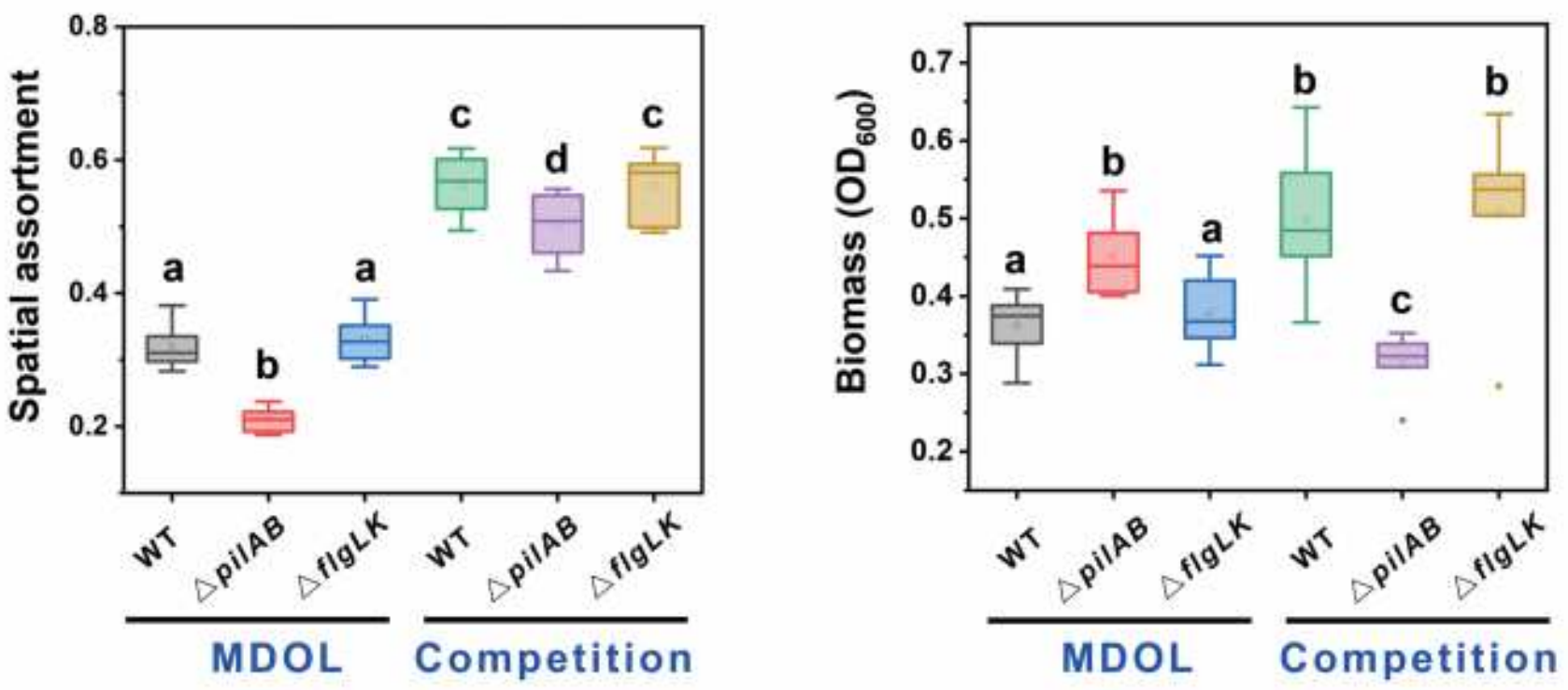

Figure 6

Type IV pili, but not flagella, are required for formation of the 'bubble' structures. (A) Images show that the colony patterning formed by the three synthetic communities in 'MDOL' scenario (supplying initial substrate salicylate as the sole carbon source) and 'Competition' scenario (supplying final product pyruvate as the sole carbon source). Typical morphology of the colony edges is zoomed in. Images were obtained after 120-h incubation. (B) Analysis of spatial assortment of the patterns formed in all conditions. As mentioned before, Assortment value closer to zero means the corresponding pattern is more mixed. (C) Analysis of community composition of the colony patterns formed in all conditions. Six replicates were performed for each condition. Different lower-case letters in (B) or (C) indicate significant difference among these conditions at 0.01 level (unpaired, two-tailed, Student's t-test). 


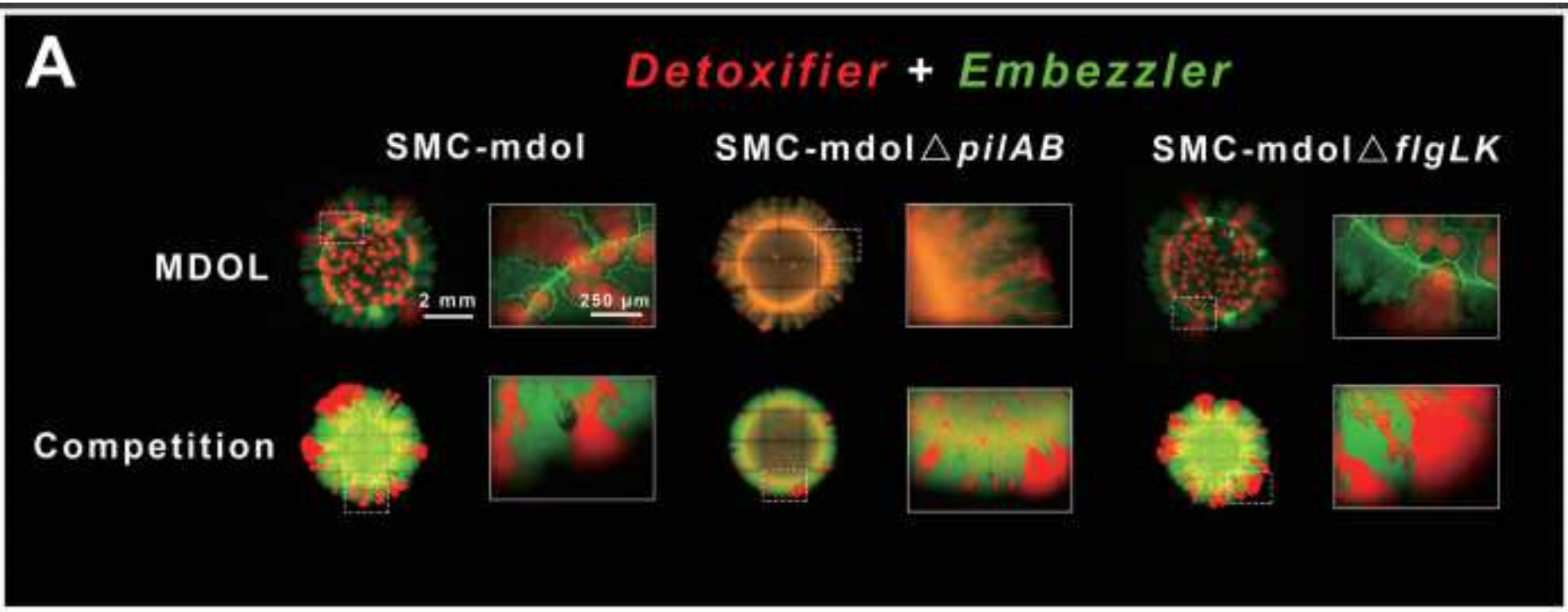

B
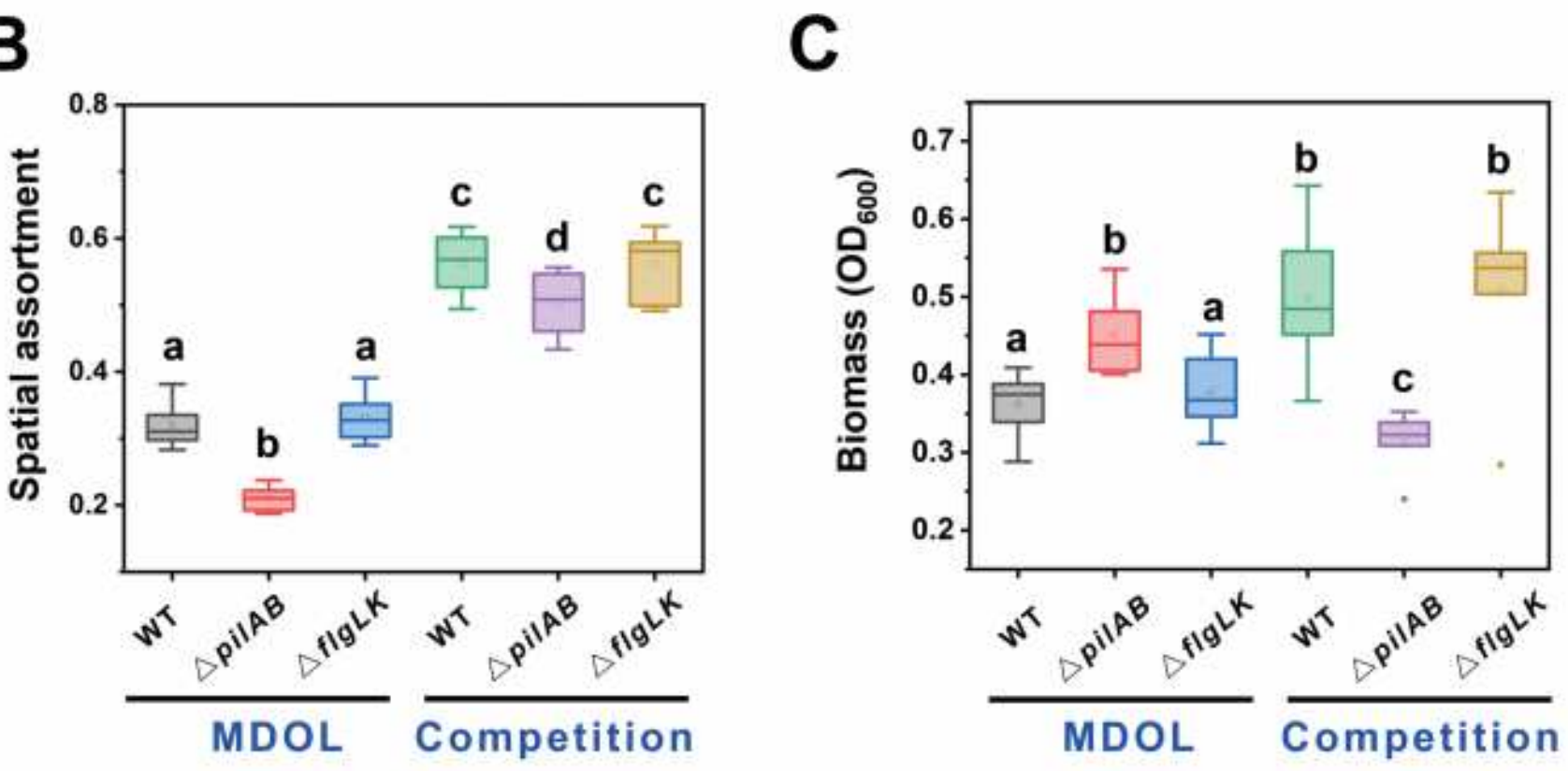

Figure 6

Type IV pili, but not flagella, are required for formation of the 'bubble' structures. (A) Images show that the colony patterning formed by the three synthetic communities in 'MDOL' scenario (supplying initial substrate salicylate as the sole carbon source) and 'Competition' scenario (supplying final product pyruvate as the sole carbon source). Typical morphology of the colony edges is zoomed in. Images were obtained after 120-h incubation. (B) Analysis of spatial assortment of the patterns formed in all conditions. As mentioned before, Assortment value closer to zero means the corresponding pattern is more mixed. (C) Analysis of community composition of the colony patterns formed in all conditions. Six replicates were performed for each condition. Different lower-case letters in (B) or (C) indicate significant difference among these conditions at 0.01 level (unpaired, two-tailed, Student's t-test). 


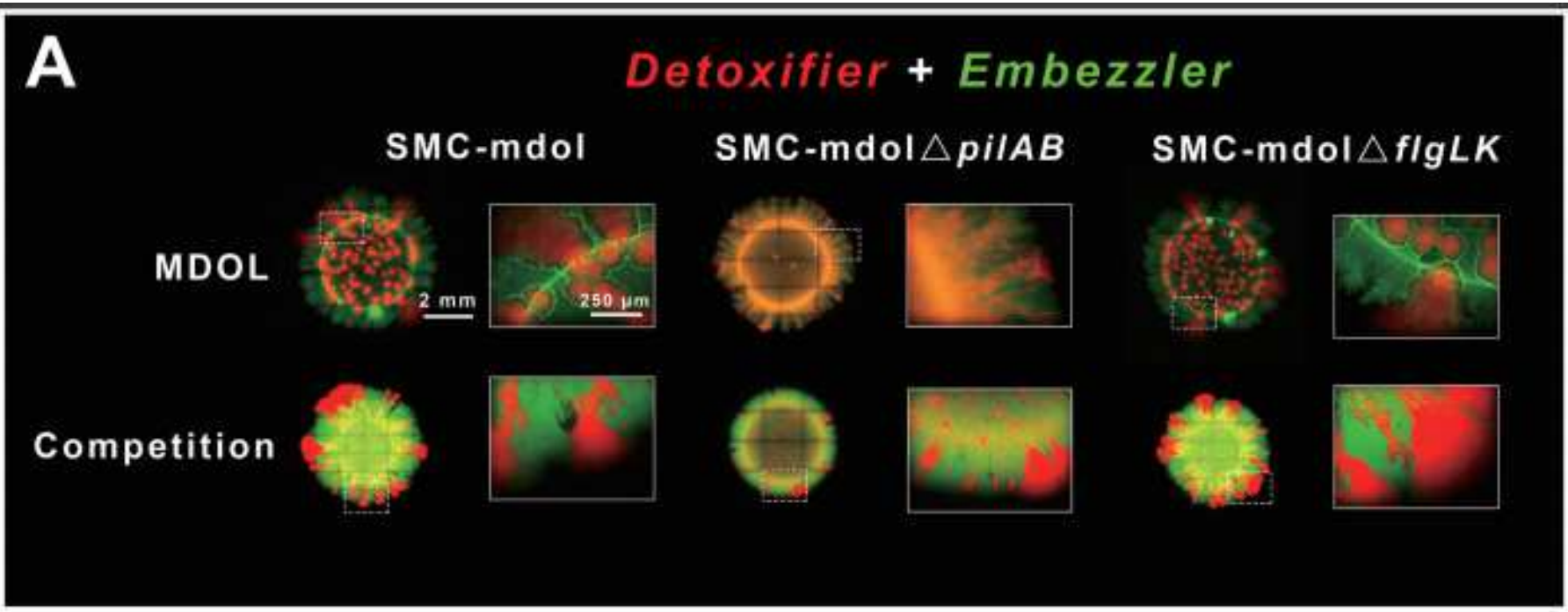

B
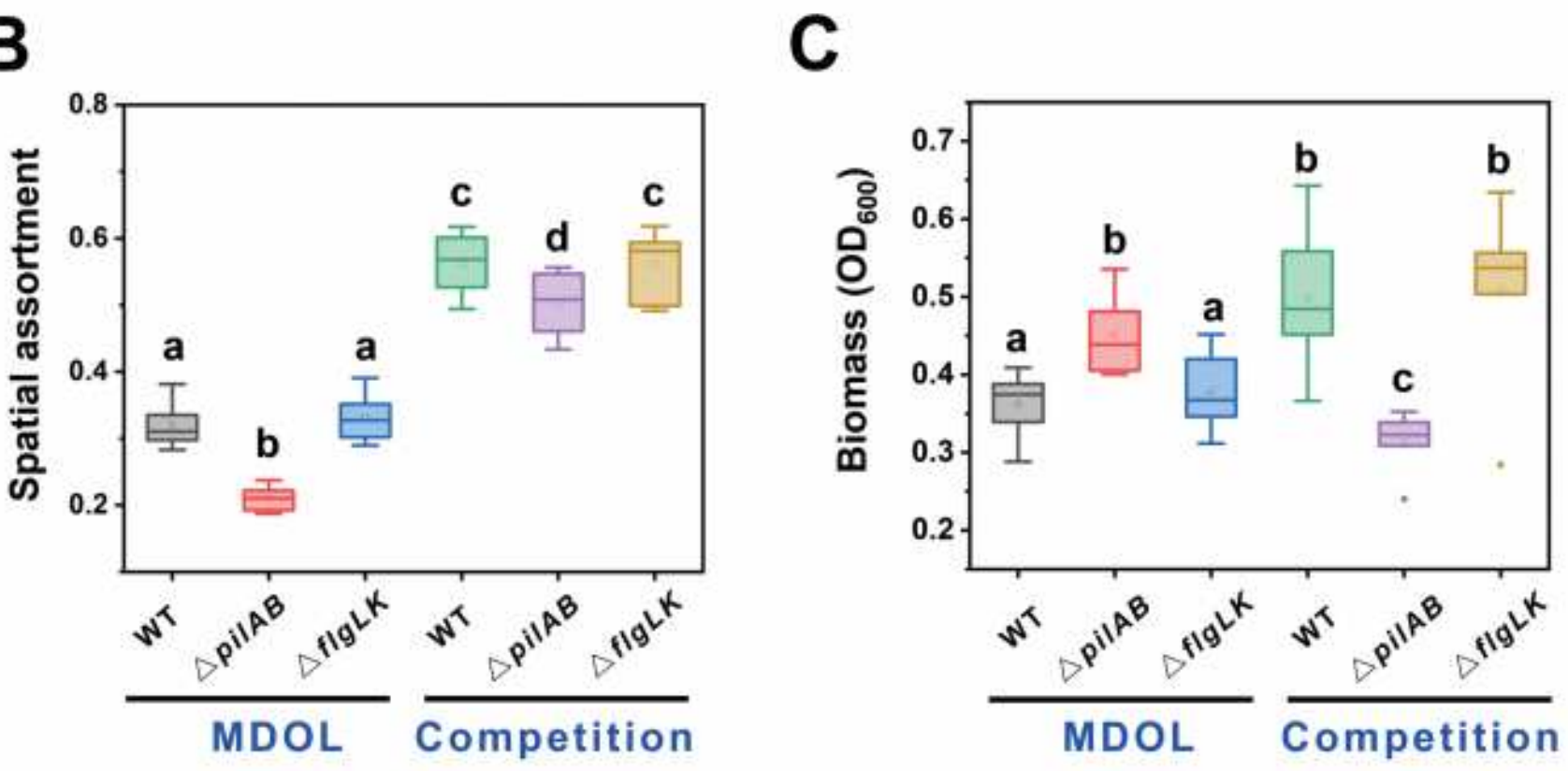

Figure 6

Type IV pili, but not flagella, are required for formation of the 'bubble' structures. (A) Images show that the colony patterning formed by the three synthetic communities in 'MDOL' scenario (supplying initial substrate salicylate as the sole carbon source) and 'Competition' scenario (supplying final product pyruvate as the sole carbon source). Typical morphology of the colony edges is zoomed in. Images were obtained after 120-h incubation. (B) Analysis of spatial assortment of the patterns formed in all conditions. As mentioned before, Assortment value closer to zero means the corresponding pattern is more mixed. (C) Analysis of community composition of the colony patterns formed in all conditions. Six replicates were performed for each condition. Different lower-case letters in (B) or (C) indicate significant difference among these conditions at 0.01 level (unpaired, two-tailed, Student's t-test). 


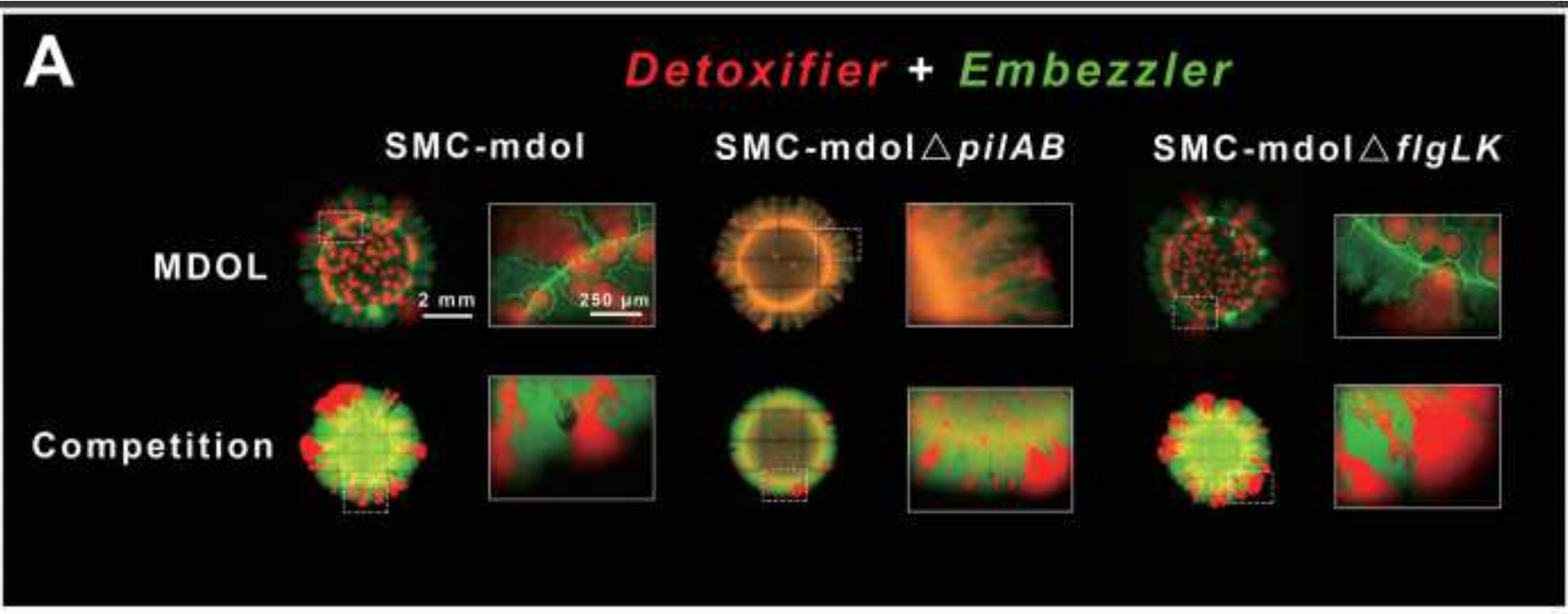

B
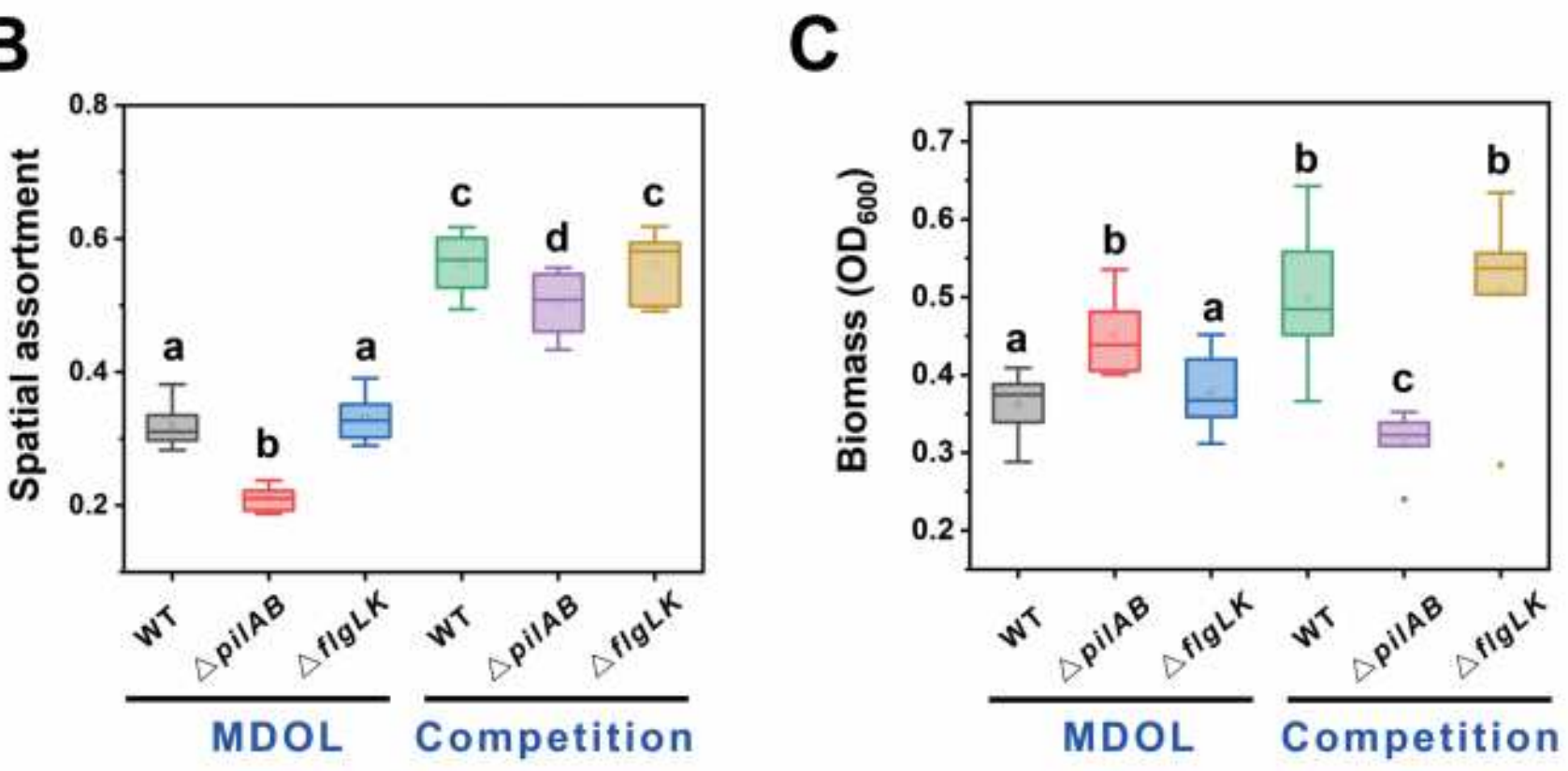

Figure 6

Type IV pili, but not flagella, are required for formation of the 'bubble' structures. (A) Images show that the colony patterning formed by the three synthetic communities in 'MDOL' scenario (supplying initial substrate salicylate as the sole carbon source) and 'Competition' scenario (supplying final product pyruvate as the sole carbon source). Typical morphology of the colony edges is zoomed in. Images were obtained after 120-h incubation. (B) Analysis of spatial assortment of the patterns formed in all conditions. As mentioned before, Assortment value closer to zero means the corresponding pattern is more mixed. (C) Analysis of community composition of the colony patterns formed in all conditions. Six replicates were performed for each condition. Different lower-case letters in (B) or (C) indicate significant difference among these conditions at 0.01 level (unpaired, two-tailed, Student's t-test).

\section{Supplementary Files}


This is a list of supplementary files associated with this preprint. Click to download.

- Supplementaryvideo1.avi

- Supplementaryvideo1.avi

- Supplementaryvideo1.avi

- Supplementaryvideo1.avi

- Supplementaryvideo2.avi

- Supplementaryvideo2.avi

- Supplementaryvideo2.avi

- Supplementaryvideo2.avi

- Supplementaryvideo3.avi

- Supplementaryvideo3.avi

- Supplementaryvideo3.avi

- Supplementaryvideo3.avi

- Supplementaryvideo4.avi

- Supplementaryvideo4.avi

- Supplementaryvideo4.avi

- Supplementaryvideo4.avi

- SupplementaryInformation20201118.docx

- SupplementaryInformation20201118.docx

- SupplementaryInformation20201118.docx

- SupplementaryInformation20201118.docx

- SupplementaryFigure1.tif

- SupplementaryFigure1.tif

- SupplementaryFigure1.tif

- SupplementaryFigure1.tif

- SupplementaryFigure2.tif

- SupplementaryFigure2.tif

- SupplementaryFigure2.tif

- SupplementaryFigure2.tif

- SupplementaryFigure3.tif

- SupplementaryFigure3.tif

- SupplementaryFigure3.tif

- SupplementaryFigure3.tif

- SupplementaryFigure4.tif 
- SupplementaryFigure4.tif

- SupplementaryFigure4.tif

- SupplementaryFigure4.tif

- SupplementaryFigure5.tif

- SupplementaryFigure5.tif

- SupplementaryFigure5.tif

- SupplementaryFigure5.tif

- SupplementaryFigure6.tif

- SupplementaryFigure6.tif

- SupplementaryFigure6.tif

- SupplementaryFigure6.tif

- SupplementaryFigure7.tif

- SupplementaryFigure7.tif

- SupplementaryFigure7.tif

- SupplementaryFigure7.tif

- SupplementaryFigure8.tif

- SupplementaryFigure8.tif

- SupplementaryFigure8.tif

- SupplementaryFigure8.tif

- SupplementaryFigure9.tif

- SupplementaryFigure9.tif

- SupplementaryFigure9.tif

- SupplementaryFigure9.tif

- SupplementaryFigure10.tif

- SupplementaryFigure10.tif

- SupplementaryFigure10.tif

- SupplementaryFigure10.tif

- SupplementaryFigure11.tif

- SupplementaryFigure11.tif

- SupplementaryFigure11.tif

- SupplementaryFigure11.tif

- SupplementaryFigure12.tif

- SupplementaryFigure12.tif

- SupplementaryFigure12.tif 
- SupplementaryFigure12.tif 